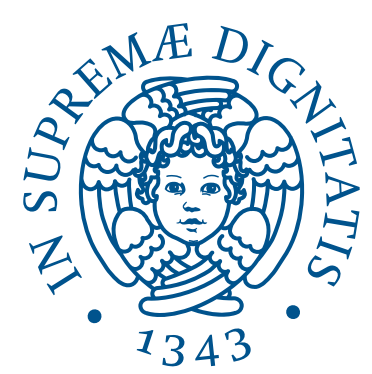

Università degli Studi di Pisa

DIPARTIMENTO DI FISICA

Corso di Laurea Magistrale in Fisica

\title{
Approaching THE CDF Top Quark MASS LEGACY MEASUREMENT IN THE LEPTON+JETS CHANNEL With the Matrix Element Method
}

Candidate:

Cecilia Tosciri
Thesis Advisor:

Prof. Giorgio Bellettini

External Advisor:

Dott. Costas Vellidis 



\section{Acknowledgments}

First, I would like to express my gratitude to my advisor, Professor Giorgio Bellettini, for giving me the great opportunity to spend a long period at Fermilab working with a group of physics experts. This opportunity made me grow as a physicist and as a person. Thanks for the support, encouragement and trust which have immensely helped me, but also for being a passionate physicist and a contagiously enthusiastic and positive person.

A very special thanks goes to my local advisor at Fermilab, Dr. Costas Vellidis, for supporting me and for being my guide over this professional experience, for his precious advices and for teaching me to be more professional and self-confident. Thank you.

This thesis work would not have been possible without the patience, advices and help of the group of experts I've worked with. So, many special thanks to Igor Suslov, Igor Volobouev, George Velev and Toni Jimenez Rugama. It has been a very rewarding experience and a real pleasure to work with you.

Moreover, I would like to thank Lina Galtieri, for considering me as a colleague since the beginning and for appreciating my dedication to the analysis. Thanks to Satyajit Behari for his help and friendliness and for the pleasing conversations. Thanks to Luigi for being a good friend and CDF mate.

Thanks to my parents, I hope to make you happy and proud.

Thanks to my beloved sister, Giulia, for being the reference point of my life and together with my brother (in-law) Pippi for protecting and helping me every time I need. Thanks to Nonna Emma, for her "forever" love.

Finally, I owe special thanks to Marco, the most extraordinary person I've ever met, for taking care of me in every moment. Thank you for being my source of energy, of love, of happiness, for profoundly changing my life. 


\section{Contents}

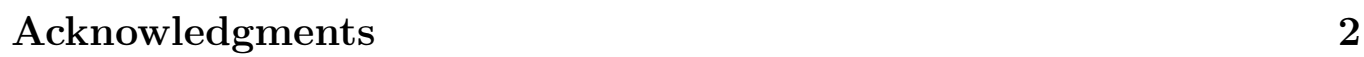

\begin{tabular}{ll}
\hline Introduction & 6
\end{tabular}

\begin{tabular}{llr}
\hline & Top Physics & 9
\end{tabular}

1.1 The Standard Model . . . . . . . . . . . . . . . . . . . . . . . 9

1.2 Top quark phenomenology . . . . . . . . . . . . . . . . . . 12

1.2 .1 Top quark production . . . . . . . . . . . . . . . 14

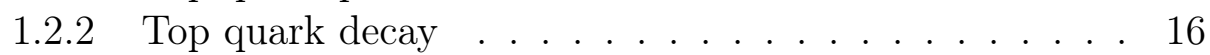

1.2 .3 Top quark mass . . . . . . . . . . . . . . . . . . . . . . 18

1.3 Previous Top Mass Measurements and Motivations . . . . . . 19

1.4 A New CDF Top Mass Measurement . . . . . . . . . . . . . . 21

2 Experimental Setup 23

2.1 The Tevatron Acceleration Complex . . . . . . . . . . . . . . . 23

2.1 .1 Proton Source . . . . . . . . . . . . . . . 23

2.1 .2 Main Injector . . . . . . . . . . . . . . . . . . 25

2.1 .3 Antiproton Source . . . . . . . . . . . . . . 25

2.1 .4 The Recycler Ring . . . . . . . . . . . . . . . . . . 26

2.1 .5 Tevatron Ring . . . . . . . . . . . . . . . . . . . . . 26

2.1 .6 Collider Performance . . . . . . . . . . . . . . . . . . . . . . . . . . . . 26

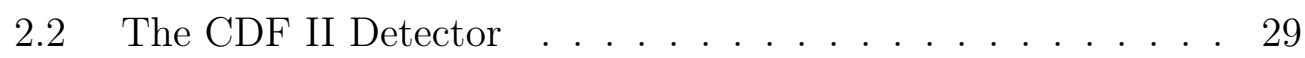

2.2 .1 The Detector Coordinate System . . . . . . . . . . . . 30

2.2 .2 The Tracking System . . . . . . . . . . . . . . . . . 31

2.2 .3 The Time of Flight system . . . . . . . . . . . . . . . . 34

2.2 .4 Calorimeters . . . . . . . . . . . . . . . . . . . 35

2.2 .5 The Muon Detector . . . . . . . . . . . . . . . . . . . 37

2.2 .6 Cherenkov Luminosity Counters . . . . . . . . . . . 38

$2.2 .7 \quad$ CDF Trigger System . . . . . . . . . . . . . . . . . . . 39 
\begin{tabular}{|ll|}
\hline 3 & Physics Object Reconstruction \\
\hline
\end{tabular}

3.1 Electron Reconstruction . . . . . . . . . . . . . . . . . . . . 42

3.2 Muon Reconstruction . . . . . . . . . . . . . . . . . . . . . . . 43

3.3 Jet Reconstruction . . . . . . . . . . . . . . . . . . . . . . . . 45

3.4 Missing Transverse Energy Reconstruction . . . . . . . . . . . . 48

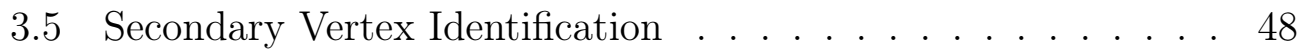

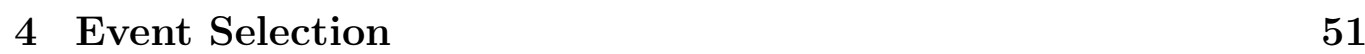

4.1 Lepton+jets topology . . . . . . . . . . . . . . . . . . . . 51

4.2 Event Signature . . . . . . . . . . . . . . . . . . . . . . . . . . 52

4.3 Background . . . . . . . . . . . . . . . . . . . . . . . 53

4.4 Monte Carlo Samples . . . . . . . . . . . . . . . . . . . . . . . 55

4.5 Selected Sample Composition . . . . . . . . . . . . . . . . 56

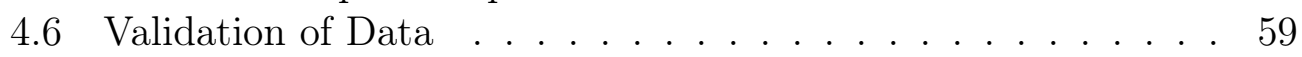

\begin{tabular}{|lll}
5 & Matrix Element Method & 69
\end{tabular}

5.1 Introduction to the Matrix Element Method . . . . . . . . . . 69

5.2 Signal Probability . . . . . . . . . . . . . . . . . 72

5.3 Background Probability . . . . . . . . . . . . . . . . 74

5.4 Matrix Element . . . . . . . . . . . . . . . . . . . . . . . . . . 74

5.5 Parton Distribution Functions . . . . . . . . . . . . . . . . . 75

5.6 Jet Energy Scale Parameter . . . . . . . . . . . . . . . . . 75

5.7 Transfer Functions $\ldots \ldots \ldots$. . . . . . . . . . . . . . 76

5.8 Normalization . . . . . . . . . . . . . . . . . . . . . 78

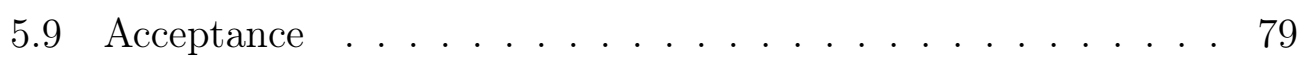

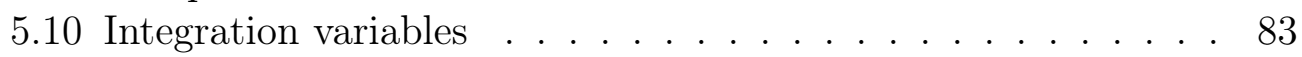

5.11 Phase Space . . . . . . . . . . . . . . . . . . . . . . . . . . . . 84

5.12 Advantages of the Matrix Element Method . . . . . . . . . . . 85

$\begin{array}{llr}6 & \text { Integral Computation } & 88\end{array}$

6.1 Integration Framework . . . . . . . . . . . . . . . . . . . . . . 88

6.2 Quasi-Monte Carlo Integration . . . . . . . . . . . . . . . . . . 89

6.3 Study of the precision of the integration . . . . . . . . . . . . 91

6.4 Calibration of Quasi-MC error . . . . . . . . . . . . . . . 96

6.5 Optimization of the integration process . . . . . . . . . . . . 98

$\begin{array}{lll}7 & \text { Next Steps } & 101\end{array}$

7.1 Top Mass Extraction . . . . . . . . . . . . . . . . . . . . . . . 101

7.2 Validation with Pseudo-Experiments . . . . . . . . . . . . . . 102

7.3 Systematic Uncertainties . . . . . . . . . . . . . . . . . . . . . 104

7.3 .1 Signal and Background Modeling . . . . . . . . . . . 105 
7.3 .2 Modeling of Detector Response . . . . . . . . . . . 107

7.3 .3 Measurement Method . . . . . . . . . . . . . . 107

\begin{tabular}{ll}
\hline Conclusions & 109
\end{tabular}

\begin{tabular}{ll}
\hline Bibliography & 111
\end{tabular} 


\section{Introduction}

The discovery of the bottom quark in 1977 at the Tevatron Collider triggered the search for its partner in the third fermion isospin doublet, the top quark, which was discovered 18 years later in 1995 by the CDF and D $\emptyset$ experiments during the Tevatron Run I. By 1990, intensive efforts by many groups at several accelerators had lifted to over $90 \mathrm{GeV} / c^{2}$ the lower mass limit, such that since then the Tevatron became the only accelerator with high-enough energy to possibly discover this amazingly massive quark. After its discovery, the determination of top quark properties has been one of the main goals of the Fermilab Tevatron Collider, and more recently also of the Large Hadron Collider (LHC) at CERN. Since the mass value plays an important role in a large number of theoretical calculations on fundamental processes, improving the accuracy of its measurement has been at any time a goal of utmost importance.

Predominantly produced in $t \bar{t}$ pairs at the Tevatron through strong interactions in $p \bar{p}$ collisions, the top quark mass was measured for the first time by CDF with a value of $176 \pm 13 \mathrm{GeV} / c^{2}$, showing that this particle was by far the heaviest known elementary particle. This has raised many questions on whether the top quark may play a special role in the Standard Model (SM), in particular in the electroweak symmetry breaking. Due to the huge mass and the very short lifetime $\left(\sim 5 \times 10^{-25} \mathrm{~s}\right)$, about 6 times shorter than the strong interaction timescale, the top quark decays weakly before hadronization into a $W$ boson and a $b$ quark, giving the chance to study the properties of a bare quark. Top quark pair events are thus characterized by the decay of their two final state $W$ bosons. This leads the $t \bar{t}$ pairs to generate the experimental signatures of two jets associated with the hadronization of the bottom quarks and either a single lepton $(e, \mu, \tau)$, one undetected neutrino and two light quark jets (lepton + jets channel), or four light quark jets (all-jets channel), or two leptons (ee, $e \mu, \mu \mu, e \tau, \mu \tau, \tau \tau)$ and two undetected neutrinos (dilepton channels). Up to now, because of its difficult experimental signatures the $\tau$ lepton was not exploited in the mass studies. 
Different approaches have been followed by the Tevatron experiments to determine the top quark mass. A very powerful technique is the Matrix Element (ME) method which determines the likelihood of observing an event under both $t \bar{t}$ and background hypotheses. The hypotheses are determined from the entire kinematic information associated to every single event by integrating the matrix element of the process over the multidimensional phase space describing the final state. For a given sample of selected events, the parameters to be measured are then determined as those values that maximize the overall likelihood. The superior statistical sensitivity of this method, with respect to other methods based on distribution-fitting, is due to the completeness of the information exploited in each event.

Since the top quark mass is a fundamental parameter of the SM, the CDF Collaboration has decided to make a major effort in order to produce its most precise measurement as a "legacy" of the experiment. A number of improvements over previous measurements are still possible as mentioned below, noticeably comparing the signal candidates not only to the signal expectation, but also to the expectation of the dominant background process $(W+j e t s)$, whose SM matrix element is now made available to the Collaboration. This thesis provides an overview of the preparatory studies to the final CDF measurement of the top quark mass. We investigate the lepton + jets channel with the full integrated luminosity of Run II $\left(9.0 \mathrm{fb}^{-1}\right)$. Our analysis uses the ME method to calculate a $t \bar{t}$ likelihood as a bi-dimensional function of the assumed top mass $m_{t}$ and $\Delta_{J E S} . \Delta_{J E S}$ parametrizes the uncertainty in our knowledge of the jet energy scale. It is a shift applied to all jet energies in units of the jet-dependent systematic error. By introducing this parameter into the likelihood, we can use as a constraint the known $W$ mass to determine the optimal $\Delta_{J E S}$ and thereby reduce the final systematic error on the measured top quark mass. For the first time in CDF analyses, we include the background ME modeling in the likelihood integration, with an expected significant reduction of the systematic error of the final result.

The massive calculations required by this double ME method imposed to develop an unconventional, less time-consuming, integration method over the phase space of the events kinematics. In order to evaluate the multidimensional integrals, we employ the "Quasi-Monte Carlo" technique, based on deterministic sequences generated by choosing points approximately equally spaced in the integration space, such that equal phase space volumes contain approximately equal number of points. This technique significantly reduces the time required to integrate an event, allowing us to reduce greatly the inte- 
gration time needed to reach the required precision. It also imposes extensive studies to make sure that no bias is introduced relative to a standard MC calculation.

The present thesis describes in detail the contributions given by the candidate to the massive preparation work needed to make the new analysis possible, during her 8 months long stay at Fermilab. These include selection of the candidates within looser cuts than in the past, estimate of the expected number of signal and background events, evaluation of the acceptance, model comparison of the final validation plots, optimization of the integration method and of its systematic error, and more as described in chapters 4 to 7 . Chapter 1 gives a brief introduction of top quark physics. Some previous mass measurements, as well as the refinements introduced in our analysis, are discussed.

Chapter 2 contains the description of the Tevatron accelerator complex and the CDF II detector.

Chapter 3 describes the reconstruction of the physical objects on which the event analysis relies.

The event selection is described in Chapter 4, where the complete list of the selection requirements and the estimation of the sample composition are presented, as well as the comparison between model and data.

Chapter 5 explains the ME method in detail, examining each part of the likelihood expression, and Chapter 6 deals with the Quasi-MC integration employed in the analysis.

In Chapter 7 the current status of the analysis and the future steps required to perform the measurement are described. Preliminarily to the final analysis of real data, future studies will include a calibration procedure and evaluation of systematic errors by means of pseudo-experiments. The goal of the measurement is to reach a total error of about $0.7 \mathrm{GeV} / c^{2}$, about $20 \%$ less than the present error of the world-averaged mass value. The candidate is planning to contribute from a distance to this final part of the measurement. 


\section{Chapter 1}

\section{Top Physics}

\subsection{The Standard Model}

The theories and discoveries of thousands of physicists since the 1930s have resulted in a remarkable insight into the fundamental structure of matter: everything in the Universe is found to be made from a few basic building blocks called fundamental particles, governed by four fundamental forces. Our best understanding of how these particles and three of the forces are related to each other is encapsulated in the Standard Model (SM) of particle physics. Developed in the early 1970s, it has successfully explained almost all experimental results and precisely predicted a wide variety of phenomena. Over time and through many experiments, the SM has become established as a well-tested physics theory.

According to the SM [66], all matter is built by a small number of fundamental spin $\frac{1}{2}$ particles called fermions: six quarks and six leptons. For each of the various fundamental constituents, its symbol and its electric charge in unit of elementary charge $e$ of the electron are given in Table 1.1 .

The leptons which carry integral electric charge include electron $e$, muon $\mu$ and tau lepton $\tau$. The neutral leptons are called neutrinos, denoted by the generic symbol $\nu$. A different flavor of neutrino is paired with each flavor of charged lepton, as indicated by the subscript.

The quarks carry fractional charges, of $+\frac{2}{3}|e|$ or $-\frac{1}{3}|e|$. In Table 1.1 the quark masses increase from left to right, just as they do for the leptons. And, just as for the leptons, the quarks are grouped into pairs, representing three generations. Particles in higher generations (having higher masses) are unstable; in order to observe and study these particles, we need to produce them via collisions with other stable particles. The quark type or flavor is 
denoted by a symbol: $u$ for $u p, d$ for down, $s$ for strange, $c$ for charmed, $b$ for bottom and $t$ for top. While leptons exist as free particles, quarks seem not to do so. It is a peculiarity of the strong forces between the quarks that they can be found only in combinations, not individually. This phenomenon is known as confinement. The combinations of quarks are called hadrons and are categorized into two families: baryons, made of three quarks, and mesons, made of one quark and one antiquark. The $u$ and $d$ quarks are the fundamental two constituents of nucleons like protons and neutrons (baryons). The electron is the most prominent lepton, as nucleons and electrons form atoms and thus the world as we see and feel it.

Table 1.1: The three generations of fundamental fermions. The charge is quoted in units of the absolute value of the electron charge.

\begin{tabular}{cccccc}
\multicolumn{5}{c}{ Generation } \\
& I & II & III & \\
\hline Particle & \multicolumn{4}{c}{ Flavor } & Charge \\
\hline \multirow{2}{*}{ Quarks } & $u$ & $c$ & $t$ & $+\frac{2}{3}$ \\
& $d$ & $s$ & $b$ & $-\frac{1}{3}$ \\
\hline \multirow{2}{*}{ Leptons } & $e$ & $\mu$ & $\tau$ & -1 \\
& $\nu_{e}$ & $\nu_{\mu}$ & $\nu_{\tau}$ & 0 \\
\hline
\end{tabular}

All experimentally observed phenomena can be described by interactions between fermions in terms of four fundamental forces: gravity, electromagnetic, weak and strong forces. The SM includes just three of these interactions. In fact, despite a strong theoretical effort to formulate a universal theory in which all the interactions are included, currently the gravitational interaction does not take its place in the model. In the SM, all particles are described as fields, and forces are interpreted as the exchange of mediator particles between interacting fermions. The force carriers are referred to as gauge bosons and are spin-1 particles. The five gauge bosons, summarized in Table 1.2 , are the $W^{ \pm}$and $Z$ (carriers of the weak force), the photon (carrier of the electromagnetic force) and the gluon (carrier of the strong force). The graviton, supposed to be the mediator of the gravitational interaction with spin-2, can not be considered as elementary particle. In fact the quantum theories with tensor interactions, like quantum gravity with a spin-2 graviton, are not renormalizable. An additional neutral scalar boson is necessary to explain electroweak symmetry breaking, i.e., the observation of non-zero 
masses of the $W^{ \pm}$and $Z$ bosons. This is the Higgs boson, the last particle predicted by the SM, recently discovered at LHC (Large Hadron Collider, CERN) by the ATLAS and CMS experiments [5|[15], after twenty years of long direct searches at the LEP (Large Electron-Positron collider at CERN) and at the Tevatron collider.

Table 1.2: SM gauge bosons: mediators of the fundamental forces between interacting elementary particles.

\begin{tabular}{ccccc}
\hline Gauge boson & Interaction & Mass $[\mathrm{GeV}]$ & Charge $\left[\frac{Q}{e}\right]$ & Spin \\
\hline Gluon, $g$ & Strong & 0 & 0 & 1 \\
Photon, $\gamma$ & Electromagnetic & 0 & 0 & 1 \\
$W^{ \pm}$ & Weak & 80.4 & \pm 1 & 1 \\
$Z^{0}$ & Weak & 91.2 & 0 & 1 \\
\hline
\end{tabular}

The SM unifies the weak and electromagnetic forces into a single electroweak interaction. The addition of Quantum Chromodynamics (QCD), which describes the strong interactions, completes the model. The resulting framework is a gauge theory, that possesses invariance under a set of local transformations, i.e., transformations whose parameters are space-time dependent, based on the gauge group $S U(3) \times S U(2) \times U(1)$, where $S U(3)$ represents the strong force, and the $S U(2) \times U(1)$ symmetry describes the electroweak interactions.

The development of the unified theory started in the 1950's by extending the gauge invariance of the electromagnetism, a well-known example of gauge theory, to the weak interaction. In order to respect the gauge invariance it was necessary to introduce a set of massless vector fields. The difficulty was that the weak interactions are known to be short range, mediated by very massive vector bosons $\left(W^{ \pm}\right.$and $Z$ ), whereas the fields of the theory were required to be massless in order to preserve gauge invariance. The apparent paradox was solved by the application of the Higgs mechanism. This is a prescription for breaking the gauge symmetry spontaneously. In this scenario one starts with a theory that possesses the required gauge invariance, but where the ground state of the theory is not invariant under the gauge transformations. The breaking of the invariance arises in the quantization of the theory, whereas the Lagrangian only contains terms which are invariant. One of the consequences of this is that the gauge bosons acquire a mass and 
the theory can thus be applied to weak interactions. The remaining step was to apply the ideas of gauge theories to the strong interaction by introducing QCD. In this theory the quarks possess an internal property called colour and the gauge transformations are local transformations between quarks of different colours. The gauge bosons of QCD are the gluons [71].

The weak force is unique among the forces in the SM as it is the only interaction which can change the flavor of quarks. In the SM, in fact, the quark mass eigenstates (physical states) do not take part as pure states in weak interactions. The unitary transformation connecting the bases of mass and weak eigenstates is represented by the Cabibbo-Kobayashi-Maskawa (CKM) matrix. By convention, the quarks with charge $+\frac{2}{3}(u, c$ and $t)$ are chosen to be pure states, and flavor mixing is described in terms of a $3 \times 3$ matrix operating on the $d, s$ and $b$ quark states [66] [3]:

$$
\left(\begin{array}{l}
d^{\prime} \\
s^{\prime} \\
b^{\prime}
\end{array}\right)=V_{C K M}\left(\begin{array}{l}
d \\
s \\
b
\end{array}\right)=\left(\begin{array}{ccc}
V_{u d} & V_{u s} & V_{u b} \\
V_{c d} & V_{c s} & V_{c b} \\
V_{t d} & V_{t s} & V_{t b}
\end{array}\right)\left(\begin{array}{l}
d \\
s \\
b
\end{array}\right)
$$

While the $d, s$ and $b$ states are eigenstates of the weak Hamiltonian, the new states $d^{\prime}, s^{\prime}$ and $b^{\prime}$ are physical states with well defined mass. The CKM matrix describes the amplitude of the mixing between quark flavors mediated by the $W$ boson. The matrix element $V_{i j}$ is the factor attached to a vertex containing a $W$ boson, an up-type quark $i$, and a down-type quark $j$. The terms in the CKM matrix are complex, and the matrix is required to be unitary in the SM; traditionally, the matrix is parameterized by three mixing angles and a CP-violating phase. All the properties of weak quark interaction are codified inside the CKM matrix.

In Figure 1.1 [72 a schematic depiction of the SM is shown.

\subsection{Top quark phenomenology}

The discovery of the bottom quark in $1977[52]$ set in motion the search for its partner in the third fermion isospin doublet. Experimental lower limits on the top mass slowly increased from a few $\mathrm{GeV}^{1}$ until the top quark was observed and its mass was directly measured at the Tevatron by CDF and $\mathrm{D} \emptyset$ experiments 18 years later [8][19]. Since its discovery in 1995, the top quark has remained in the focus of particle physics research. With a mass of

\footnotetext{
${ }^{1}$ Here and in the following we are going to use natural units.
} 


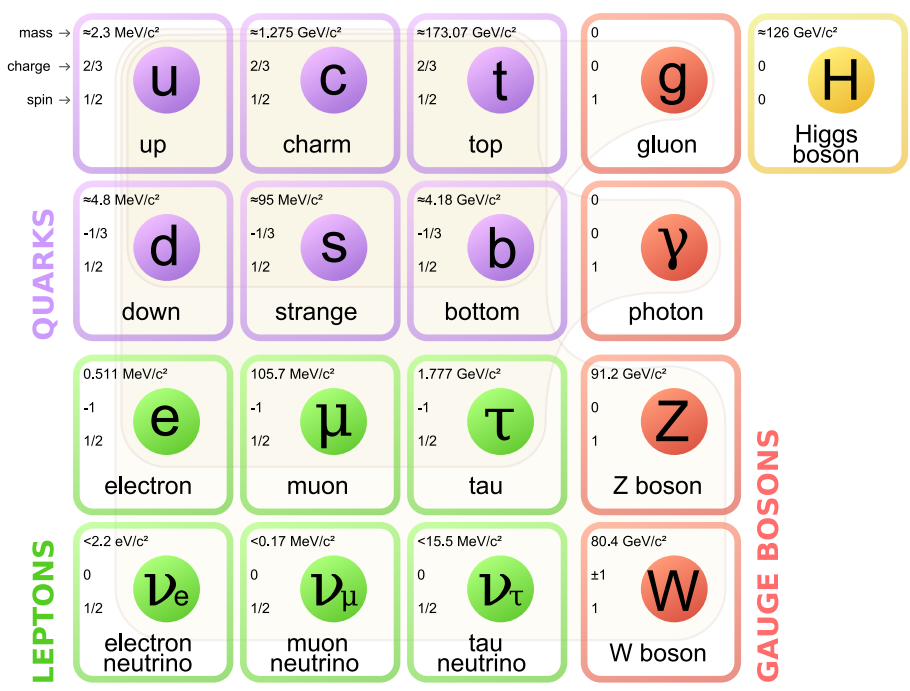

Figure 1.1: The SM of elementary particles, with the three generations of matter, gauge bosons in the fourth column, and the Higgs boson in the fifth [72].

$m_{t}=173.34 \pm 0.27 \pm\left. 0.71 \mathrm{GeV}[21]\right|^{2}$, it is by far the heaviest of all known quarks. This has posed many questions whether the top quark may play a special role in the SM, in particular in the electroweak symmetry breaking.

Top quark phenomenology is driven by its large mass. Being heavier than a $W$ boson, it is the only quark that decays semi-weakly, i.e., into a real $W$ boson and a $b$ quark, with a very short lifetime. Quarks and gluons created in high energy collisions cannot exist individually, because of color confinement, but they combine with other quarks and antiquarks to form hadrons. The process of the formation of hadrons out of quarks and gluons is called hadronization. Due to its very short lifetime, the top quark decays before hadronization can occur. In addition, it is the only quark whose Yukawa coupling to the Higgs boson is order of unity. Its exclusive feature provides a unique laboratory where our understanding of the strong interactions, both in the perturbative and non perturbative regimes, can be tested. An accurate knowledge of its properties (mass, couplings, production cross section, decay branching ratios, etc.) can bring key information on fundamental interactions at the electroweak breaking scale and beyond [32].

\footnotetext{
${ }^{2}$ This is the current resulting combination of the latest top mass measurements performed by the CDF and $\mathrm{D} \emptyset$ at the Tevatron and by ATLAS and CMS at the LHC [32].
} 

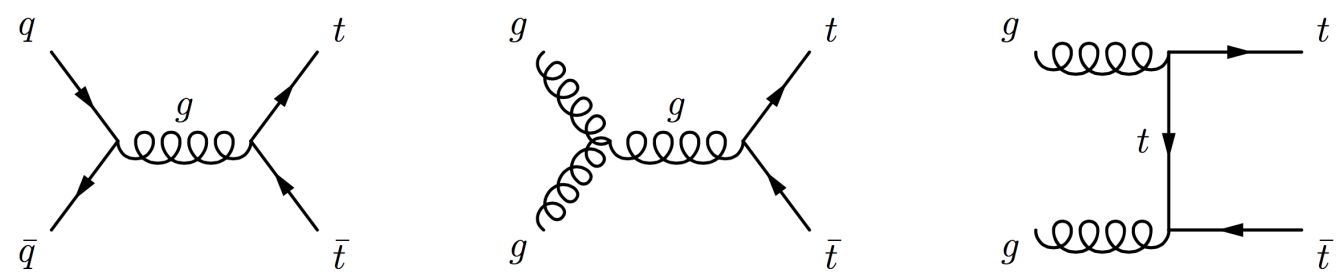

Figure 1.2: Some of the Feynman diagrams that contribute to $t \bar{t}$ production, through annihilation of quarks (left diagram) and fusion of gluon (central and right diagrams).

\subsubsection{Top quark production}

In hadron collisions, top quarks are produced dominantly in pairs through the strong processes $q \bar{q} \rightarrow t \bar{t}$ and $g g \rightarrow t \bar{t}$, which can be calculated in perturbative QCD. Figure 1.2 illustrates some of the leading order (LO) diagrams that contribute to the $t \bar{t}$ production. Approximately $85 \%$ of the production cross section at the Tevatron $(\sqrt{s}=1.96 \mathrm{TeV})$ is from $q \bar{q}$ annihilation, with the remainder from gluon-gluon fusion, while at LHC energies, about $90 \%$ of the production is from the latter process at $\sqrt{s}=14 \mathrm{TeV}(\approx 80 \%$ at $\sqrt{s}=7$ $\mathrm{TeV})$. This is due to the different dependence on collision energy of quark and gluon structure functions at the appropriate momenta for generating the top quark mass.

In hadron collisions the hard scattering takes place between protons and (anti-)protons ( $p \bar{p}$ collisions at the Tevatron, $p p$ collisions in the case of LHC). According to the SM, the proton is made up of three valence quarks, a sea of virtual quarks and antiquarks (sea quarks) that surround them, and gluons which bind them together. Since the proton cannot be treated perturbatively, only the hard scattering process between partons is considered. The remaining partons are treated as spectators.

The $t \bar{t}$ production process is explained by the parton model of the hard scattering process: two partons, one of each colliding (anti-)proton, take part in the interaction, carrying momentum fractions $x_{1}, x_{2}$ of the incoming hadron momenta. These fractions are unknown on an event-by-event basis. The probability density $f_{i}\left(x_{i}, Q^{2}\right)$ for a parton with flavor $i$ to participate in the hard scattering interaction with momentum fraction $x_{i}$ and factorization scale $Q^{2}$ is referred to as the Parton Distribution Function (PDF). The shapes of the PDFs are extracted from global QCD fits at next-to-leading order (NLO) from data. Given the PDFs, the total cross section of a process $p_{A} p_{B} \rightarrow p_{1} p_{2}$ can be approximated by summing over all possible parton interactions and 

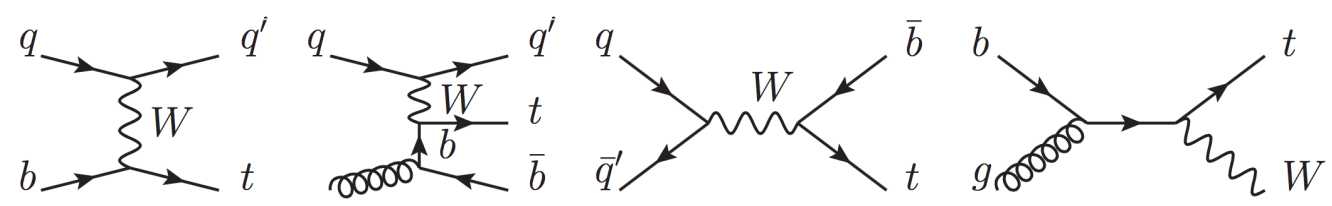

Figure 1.3: Example Feynman diagrams for single top quark production at LO QCD. From left to right: $t$-channel production as flavor excitation and as $W$-gluon fusion, $s$-channel production, $t W$-channel production.

weighting each by its probability:

$$
\sigma=\sum_{A, B} \int d x_{A} d x_{B} f_{A}\left(x_{A}, Q^{2}\right) f_{B}\left(x_{B}, Q^{2}\right) \hat{\sigma}\left(p_{A} p_{B} \rightarrow p_{1} p_{2}\right)
$$

The cross section is calculated at next-to-next-to leading order (NNLO). Assuming a top quark mass of $173.3 \mathrm{GeV}$, close to the Tevatron+LHC average, the resulting theoretical prediction of the top quark pair cross section at the Tevatron at $\sqrt{s}=1.96 \mathrm{TeV}$ is $\sigma_{t \bar{t}}=7.16_{-0.20-0.12}^{+0.11+0.17} \mathrm{pb}$, where the first uncertainty is from scale dependence and the second from the PDFs [32].

The pair production cross section measurement, obtained by combining six measurements of the inclusive top quark pair production cross section from data collected with the $\mathrm{CDF}$ and $\mathrm{D} \emptyset$ detectors, returns the value $\sigma_{t \bar{t}}=7.60 \pm 0.41 \mathrm{pb}$ for a top quark mass of $m_{t}=172.5 \mathrm{GeV}[12]$. The data correspond to integrated luminosities of up to $9.0 \mathrm{fb}^{-1}$. This result is in good agreement with the theoretical prediction.

Top quarks can also be produced singly via the electroweak interaction. There are three possible channels of single-top production: $s$-channel, $t$-channel and associated $t W$ production, as illustrated in Figure 1.3. These mechanisms lead to a smaller cross section. Approximate NNLO cross sections are calculated for the various single-top production channels given an input top mass. For $m_{t}=173 \mathrm{GeV}$ in $p \bar{p}$ collisions at $\sqrt{s}=1.96 \mathrm{TeV}$ the cross section obtained is $1.04_{-0.02}^{+0.00} \pm 0.06 \mathrm{pb}$ for $t$-channel and $0.523_{-0.005-0.028}^{+0.001+0.030} \mathrm{pb}$ for $s$-channel production [59]. The production in $W t$ channel results negligible at the Tevatron.

In our analysis we will measure the top quark mass studying pair production. 


\subsubsection{Top quark decay}

In the SM the top quark decay rates in down-type quaks $(d, s, b)$ are proportional to the $\left|V_{t(d, s, b)}\right|^{2}$, the squared CKM matrix element that relates the top and the down-type quark. The lower generation terms $V_{t b}$ and $V_{t s}$ being very small, the unitarity of the CKM matrix constrains the $\left|V_{t b}\right|$ element to be very close to unity. A recent measurement of the magnitude of the top-to-bottom quark coupling has been obtained from the combination of $\mathrm{CDF}$ and $\mathrm{D} \emptyset$ results in the measurement of cross sections for single-top quark production [13], returning the value $\left|V_{t b}\right|=1.02_{-0.31}^{+0.29}$. Consequently, being $\left|V_{t d}\right|$ and $\left|V_{t s}\right|$ of the order of $10^{-3}$ and $10^{-4}$ respectively, the fraction of top quark decay branching ratios is:

$$
R=\frac{B(t \rightarrow W b)}{B(t \rightarrow W q)}=\frac{\left|V_{t b}\right|^{2}}{\left|V_{t d}\right|^{2}+\left|V_{t s}\right|^{2}+\left|V_{t b}\right|^{2}} \approx 1 .
$$

In other words, the top quark decays into a $\mathrm{W}$ boson and a $b$ quark $\left(t \bar{t} \rightarrow W^{+} b W^{-} \bar{b}\right)$ nearly $100 \%$ of the time.

Due to this property and to the large mass, the top quark appears to have a large width. The LO width is:

$$
\Gamma_{t}^{0}=\frac{\left|V_{t b}\right|^{2} G_{F} m_{t}^{3}}{8 \pi \sqrt{2}} .
$$

where $G_{F}$ is the Fermi coupling constant and $m_{t}$ refers to the top quark pole mass which will be discussed in the next section 1.2.3. Assuming the $b$ quark to be massless and neglecting higher order terms, the total decay width $\Gamma_{t}$ at NLO of the top quark can be approximated as

$$
\Gamma_{t}=\Gamma_{t}^{0}\left(1-\frac{m_{W}^{2}}{m_{t}^{2}}\right)\left(1+2 \frac{m_{W}^{2}}{m_{t}^{2}}\right)\left[1-\frac{2 \alpha_{s}}{3 \pi}\left(\frac{2 \pi^{2}}{3}-\frac{5}{2}\right)\right],
$$

where $m_{W}$ is the mass of $W$ boson and $\alpha_{s}$ the strong coupling parameter, running with the energy scale $Q^{2}, \alpha_{s}\left(Q^{2}\right)$. In the top quark phenomenology $\alpha_{s}$ is typically taken at the highest point where it is experimentally determined, i.e. the $Z$ pole mass $m_{Z}=91 \mathrm{GeV}$. The width for a value of $m_{t}=173.3 \mathrm{GeV}$ is $1.35 \mathrm{GeV}$. Thus, the top width is significantly larger than the typical QCD scale $\Lambda_{Q C D} \approx 200 \mathrm{MeV}$. As a result, the top lifetime $\tau_{t} \approx 0.5 \times 10^{-24} \mathrm{~s}$ is much smaller than the typical time for formation of QCD bound state hadrons $\tau_{Q C D} \approx 3 \times 10^{-24} \mathrm{~s}$. Therefore, the top quark decays before top-flavored hadrons or $t \bar{t}$-quarkonium bound states can form.

The final states for the pair production process can be divided into classes depending on the decays of the two $W$ bosons in the event. Evidently, the 
quarks produced in $W$ decays are not observed as such, but they hadronize producing jets of particles which are subsequently observed and measured in the detector.

Different experimental signatures of the $t \bar{t}$ decay are classified as follows:

- Dilepton events, where both $W$ bosons decay into an $e \nu$ or $\mu \nu$ final state, are characterized by two energetic, isolated leptons, two energetic $b$ jets, and two undetected neutrinos. Neutrinos escape direct detection but their presence can be inferred by an excess of missing transverse energy (MET) in the event. Because of the presence of two high-energy leptons, this analysis channel provides the cleanest signature and offers a good signal-to-background ratio. However, occurring in $\sim 7 \%$ of cases (this is the value of the branching ratio obtained including $\tau$ leptons coming from $W$ decays), it has the lowest branching ratio. Furthermore, because of the two neutrinos, the kinematics of the event are underconstrained.

- Fully hadronic events, where both $W$ bosons decay hadronically. The signature is six jets, two of which are $b$ jets, and no significant missing transverse energy. Though this channel enjoys $\sim 55 \%$ of the decay rate and allows for the full reconstruction of the event kinematics, it is the most difficult to study because of the large QCD multijet background. Therefore sophisticated techniques are necessary to improve the signal to background ratio.

- Lepton+Jets events, where one $W$ boson decays hadronically and the other into an $e \nu$ or $\mu \nu$ final state, are characterized by an energetic isolated lepton, four energetic jets, and missing transverse energy from one undetected neutrino. With only one neutrino, the event can be kinematically reconstructed. This analysis channel accounts for $\sim 38 \%$ ( $\tau$ events included) of the decay rate while providing a distinct experimental signature through the isolated lepton.

Particular attention is required for $\tau$ events, where at least one $W$ boson decays into a $\tau \nu$ final state. In fact, while $W \rightarrow l \nu_{l}$ decays, with $l=e$ or $\mu$, result in unambiguous experimental signatures, the case $W \rightarrow \tau \nu_{\tau}$ is more complicated as tau leptons decay in a variety of ways. Depending on its decay, the $\tau$ lepton can be identified as a narrow jet, an isolated track, or an electron or muon. Two energetic $b$ jets, missing transverse energy, and the decay products from the second $\mathrm{W}$ boson complete the topology. Explicit identification of $\tau$ leptons is a major challenge at hadron colliders. However, events in which a $\tau$ decays into an electron or muon will appear in 
the electron/muon + jets signal samples. Top quark pairs decay modes and their branching ratios are illustrated in Figure 1.4 .

Our measurement is performed in the lepton + jets channel, as it offers the best balance between a clean signature and a good branching ratio. We also include the cascade decay of $W \rightarrow \tau \nu$ and $\tau \rightarrow e \nu$ or $\tau \rightarrow \mu \nu$.

\section{Top Pair Decay Channels}

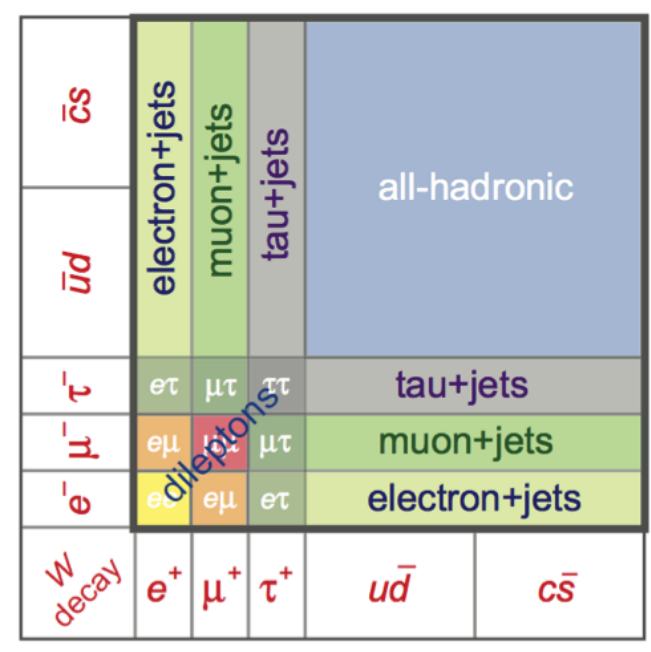

Figure 1.4: The graphic represents all the $t \bar{t}$ decay modes. The areas are proportional to the branching ratios [67].

\subsubsection{Top quark mass}

Unlike the leptons, quarks are confined inside hadrons and are not observed as physical particles. Quark masses therefore cannot be measured directly, but must be determined indirectly through their influence on hadronic properties. Any quantitative statement about the value of a quark mass must make careful reference to the particular theoretical framework that is used to define it. For example, in the overall fit of electroweak measurements the top quark mass needs to be expressed in the $\overline{M S}$ renormalization scheme [33]|58|. What is being measured in $t \bar{t}$ production experiments is the Monte Carlo-defined mass with whatever Monte Carlo (MC) is used to measure it, because the top mass is always determined from the data through the fit of some mass-dependent $\mathrm{MC}$ prediction. For typically used parton-shower MC generators, this mass is close to the pole mass rather than the "bare" mass defined in a renormalization scheme such as the $\overline{M S}$ one. The pole mass is defined by analogy with the 
mass definition used in most QED calculations. Conceptually, one imagines taking the particle to infinity and measuring its classical mass in isolation. Even though this cannot be physically done for a quark in QCD, one can give an operational definition at any finite order of perturbation theory, with the mass parameter defined as the real part of the position of the pole in the complex momentum space:

$$
\frac{i}{\not p-m_{\text {pole }}} \text {. }
$$

\subsection{Previous Top Mass Measurements and Mo- tivations}

The last world combination of measurements of the top quark mass has been performed in 2014 between the results of CDF and D $\emptyset$ at the Tevatron and the ATLAS and CMS experiments at the LHC. The resulting combined measurement, with a precision of $0.4 \%$, is [21

$$
m_{t}=173.34 \pm 0.27 \text { (stat) } \pm 0.71 \text { (syst) } \mathrm{GeV} .
$$

Figure 1.5 summarizes the input measurements and the results of the combination. However, this scenario is now incomplete because it does not include two more recent and very precise results:

$$
\begin{gathered}
m_{t}(\mathrm{D} \emptyset)=174.98 \pm 0.58(\text { stat }) \pm 0.49(\text { syst }) \mathrm{GeV} \\
m_{t}(\mathrm{CMS})=172.44 \pm 0.13(\text { stat }) \pm 0.47(\text { syst }) \mathrm{GeV}
\end{gathered}
$$

The top mass value in $(1.8)$ is the latest result obtained by $\mathrm{D} \emptyset$ using matrix element technique and jet energy scale in situ calibration in lepton + jets final states of the full Run II sample [20]. The second mass value, displayed in (1.9), is the result based on the proton-proton data recorded by the CMS experiment at the LHC at $\sqrt{s}=8 \mathrm{TeV}$, in the combined lepton + jets, all-jets and dilepton decay channels, by using the ideogram method [14]. These results represent the most precise measurements ever obtained for the top quark mass. However, there is a significant discrepancy between them. With this new top mass measurement, CDF aims at improving considerably the systematic uncertainty as well as the statistical weight of the events and the statistics itself, exploiting the full CDF Run II dataset. Hopefully, the new measurement will help solving the current tension between the two latest measurement listed above. 


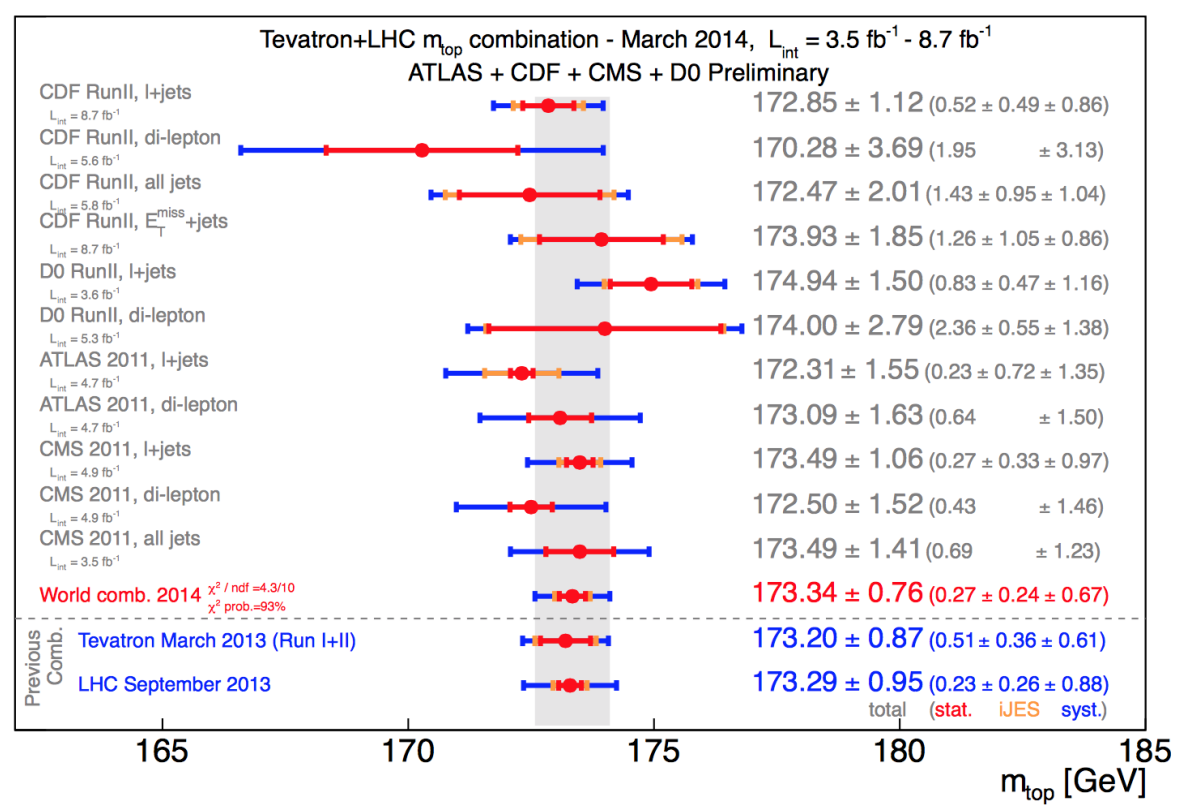

Figure 1.5: Input measurements and result of their combination, compared (lowest lines) to Tevatron combined and LHC combined $m_{t}$ values. The red lines correspond to the statistical uncertainty while the blue lines show the total uncertainty. While in the Tevatron experiments the statistical error dominates, at the LHC the error is still mostly systematic.

Over and above this motivation, there are important reasons because experimentalists pay specific attention and spend much effort in measuring the top mass with very high precision. The large value of $m_{t}$ implies a large coupling to the Higgs boson. The top quark Yukawa coupling $y_{t}=m_{t} / v$ where $v \sim 246 \mathrm{GeV}$ is the vacuum expectation value of the Higgs field, is close to unity. Because of this observation, it has often been speculated that the top quark may play a special role in the electroweak symmetry breaking, either in the context of the Higgs model, or invoking alternative mechanisms through which elementary particles acquire mass. The top quark appears in higher order loop diagrams of the electroweak theory, which implies that $m_{t}$ is a crucial parameter in this theory. Precise measurements of $m_{t}$ provide, together with other parameters of the electroweak theory, in particular the mass of the $W$ boson $m_{W}$, indirect constraints on the mass of the Higgs boson. All together, the value of the top mass plays an important role in precision physics of the Higgs sector. Some of the lowest order diagrams that link $m_{t}, m_{W}$ and $m_{H}$ are shown in Figure 1.6 .

The most recent $m_{H}$ measurements indicate a good agreement between 

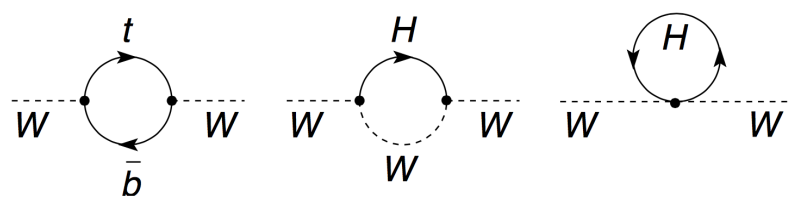

Figure 1.6: Lowest order diagrams that correlate $m_{t}, m_{W}$ and $m_{H}$.

predicted and observed mass values, as shown in Figure 1.7 [1]. In this plot, the diagonal gray lines indicate the predicted value of the Higgs mass, given those of the $W$ boson and top quark and various electroweak parameters (such as coupling constants, mixing angles, $\mathrm{CP}$ phases, and other quark masses). The horizontal and vertical green bands indicate the 1-standard deviation $(\sigma)$ confidence regions of the measured $W$ boson and top quark masses; the oval green contours cover $1 \sigma$ and $2 \sigma$ confidence areas for the joint $W$ boson and top quark masses. The blue contours are $1 \sigma$ and $2 \sigma$ confidence areas for the $W$ boson and top quark masses as predicted from electroweak parameters and the measured mass of the Higgs boson. The remarkable agreement between the experimental measurements and the predictions indicates a profound solidity of the SM.

Besides its potential role in electroweak symmetry breaking, the top quark plays an important role in many scenarios for new physics beyond the SM. This constitutes one of the main motivations for the top quark physics today's program at the LHC. Several models predict the existence of new particles which decay predominantly into top quark pairs. Therefore, it is attractive to search for resonances in the top quark pair invariant mass distribution. New particles may also be produced in top quark decays.

\subsection{A New CDF Top Mass Measurement}

In this context, a new CDF top mass measurement is inserted. CDF has completed its program of top mass measurements with the template method (see section 5.12 for a brief description of the method) using the full luminosity in all four channels (dilepton, lepton + jets, MET + jets, alljets), with a remarkable total precision of the average result close to $0.7 \%$. With the new measurement we are going to exploit all the advantages of the Matrix Element method, which is the most powerful tool available for such measurement, as widely explained in the following chapters of this thesis. We use the full Run II dataset collected by CDF between 2001 and $2011\left(9.0 \mathrm{fb}^{-1}\right)$, looking at the lepton + jets final states. This channel has been already used in the previous CDF template measure with the full luminosity [9], returning 


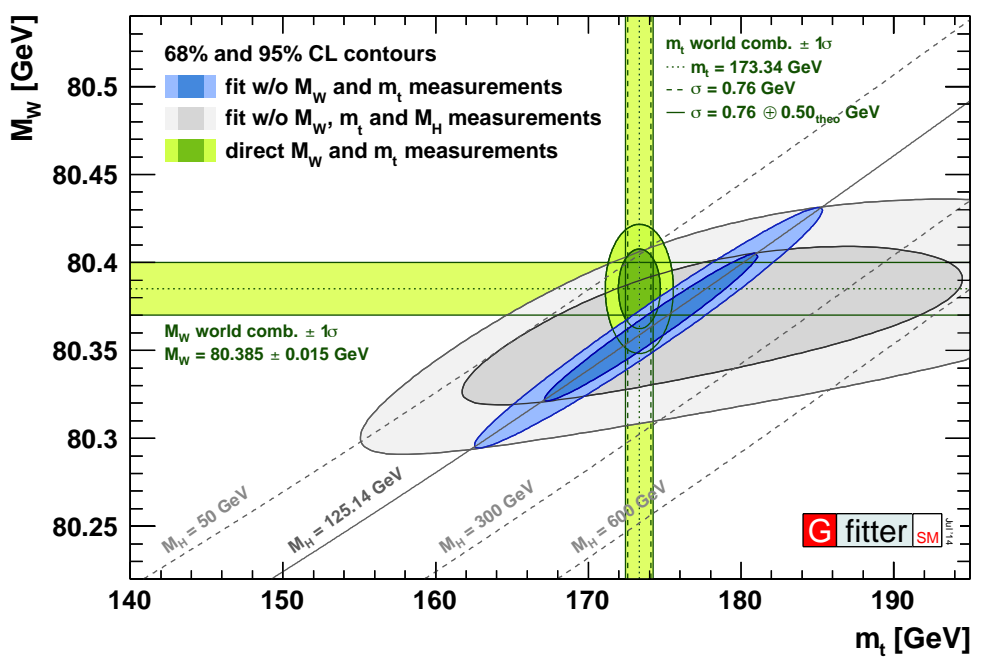

Figure 1.7: The masses of the top quark, $W$ boson, and Higgs boson are related by the SM. In this plot, the diagonal gray lines indicate the predicted value of the Higgs mass, given those of the $W$ boson and top quark. The horizontal and vertical green bands indicate $1 \sigma$ confidence regions of the measured $W$ boson and top quark masses; the oval green contours cover $1 \sigma$ and $2 \sigma$ confidence areas for the joint $W$ boson and top quark masses. The blue contours are $1 \sigma$ and $2 \sigma$ confidence areas for the $W$ boson and top quark masses as predicted from electroweak parameters and the measured mass of the Higgs boson.

the value $m_{t}=172.85 \pm 0.71($ stat $) \pm 0.85$ (syst) $\mathrm{GeV}$.

We use as a reference for our analysis the previous CDF top mass measurement performed on a dataset of $5.6 \mathrm{fb}^{-1}$ of integrated luminosity by using the Matrix Element method, with a resulting value $m_{t}=173.0 \pm 0.9$ (stat $) \pm$ 0.9 (syst) $\mathrm{GeV}$. However, in our analysis we introduce several upgrades:

- beyond the increase of the integrated luminosity, we include new sample categories like the untagged category and loose category, i.e., reduced $p_{T}$ jet cut category (which will be explained in chapter 4 ), to improve the statistics of the measurement;

- we include for the first time in CDF analyses the background Matrix Element modeling in the likelihood integration (this is the main and the most complex improvement of the analysis);

- we are going to obtain smaller systematic uncertainties on the final measurement by introducing several new signal and background MC modeling, as well as new detector modeling. 


\section{Chapter 2}

\section{Experimental Setup}

\subsection{The Tevatron Acceleration Complex}

The Tevatron [25] 24 was a circular particle accelerator in the United States, located at the Fermi National Accelerator Laboratory (FNAL, also known as Fermilab), just east of Batavia, Illinois, which operated from 1983 until the end of September 2011. The Tevatron was a synchrotron where proton-antiproton $(p \bar{p})$ collisions were accelerated and stored at a centerof-mass energy of $\sqrt{s}=1.96 \mathrm{TeV}$. The final proton and antiproton beams were the result of a complex acceleration system which involved different stages, spanning from proton and antiproton production, their acceleration and transfer across different sub-systems, to their actual collision in designated interaction points. The interaction points were located at the optics points $\mathrm{B} \emptyset$, where the Collider Detector at Fermilab (CDF) was situated and D $\phi$, where the homonymous detector was located. A schematic view of the Tevatron acceleration chain is shown in Figure 2.1.

\subsubsection{Proton Source}

The Proton Source consists of the Pre-Accelerator, the Linac, and the Booster. The Pre-Accelerator [24] is a Cockcroft-Walton electrostatic chamber in which $H^{-}$gas is produced by electron-capturing by hydrogen ionization. $H^{-}$ions are accelerated by a positive voltage to a kinetic energy of $750 \mathrm{KeV}$ and injected through a transfer line to the linear accelerator, the Linac. The Linac [28] accelerates $H^{-}$ions from $750 \mathrm{keV}$ to $400 \mathrm{MeV}$. The Booster is the next level of acceleration. At the booster entrance, the electrons are stripped-off the $\mathrm{H}^{-}$ions leaving bare protons to enter the accelerator.

The Booster 27] is a synchrotron of $75 \mathrm{~m}$ radius where protons are accelerated to $8 \mathrm{GeV}$ and transferred into the Main Injector. 


\section{FERMILAB'S ACCELERATOR CHAIN}

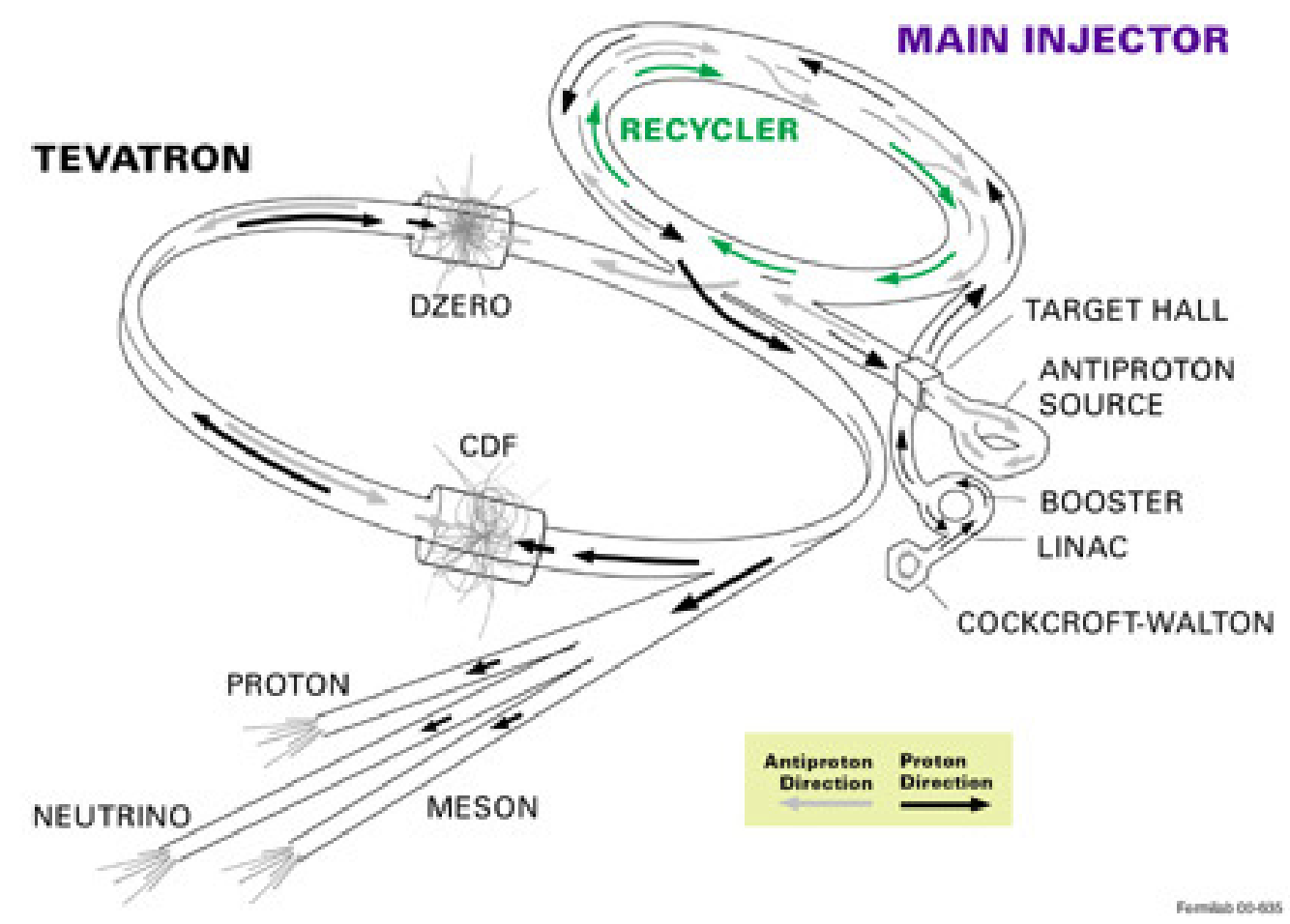

Figure 2.1: Layout of the Fermilab accelerator complex. Protons (black arrow) are accelerated at the Cockcroft-Walton, Linac, Booster, Main Injector and finally at the Tevatron. The anti-protons (grey arrow) from the anti-proton source are first accelerated at the Main Injector and then at the Tevatron. 
At the end of the acceleration process in the main injector, the proton high energy shot ("batch") is a sequence of 84 bunches containing approximately $6 \times 10^{10}$ protons each, distant from each other by $18.9 \mathrm{~ns}$.

\subsubsection{Main Injector}

The Main Injector (MI) 29] is a circular synchrotron seven times the circumference of the Booster and slightly more than half the circumference of the Tevatron. In its normal use during when the Tevatron was in operation, it accelerated $8 \mathrm{GeV}$ protons from the Booster to either $120 \mathrm{GeV}$ or $150 \mathrm{GeV}$, depending on their destination. When used to inject into the Tevatron, the final beam energy was $150 \mathrm{GeV}$. When used to produce antiprotons, the final energy was $120 \mathrm{GeV}$. As well as accepting protons from Booster, the MI could also accept antiprotons back from the Recycler and accelerate them to 150 $\mathrm{GeV}$ for the injection in the Tevatron. Protons extracted from the Booster were collected in bunches, as well as antiprotons coming from the Recycler. Thirty-six bunches accelerated by the MI were needed to fill the Tevatron with protons and antiprotons.

\subsubsection{Antiproton Source}

The Antiproton Source [26] was a very expensive component of the Tevatron Collider complex. Considering that no other research program with antiproton beams could justify the cost of maintaining it in operation, it was shut down together with the Tevatron collider. At present, the only program exploiting antiproton beams of very low energy is continued at CERN.

The Antiproton Source was composed by three main parts: the Target Station, the Debuncher, and the Accumulator. To produce antiprotons, 120 $\mathrm{GeV}$ proton beams with an intensity up to $\sim 10^{12}$ are steered onto a nickel target. The collisions created a wide range of secondary particles, among which are antiprotons, that are produced with an efficiency of about $18 \bar{p} / 10^{6} p$. Downstream of the target station is located the collection lithium lens module, in which a solenoidal magnetic field focuses the negative secondaries. The purpose of the pulsed dipole magnet following the lens is to select $8 \mathrm{GeV}$ negative charged particles and to force them towards the Debuncher, where the momentum spread is reduced using stochastic and momentum cooling. The Debuncher and Accumulator were small rings sharing the same tunnel. Single shots were received and cooled in the small acceptance Debuncher ring and transferred in the Accumulator where large current could be stored. The 
$8 \mathrm{GeV}$ stores were further stochastically cooled as needed before transferring into the Recycler.

\subsubsection{The Recycler Ring}

When the collected antiprotons had saturated the Accumulator acceptance $\left(\sim 6 \times 10^{11}\right.$ antiprotons), they were transferred to the Recycler [30|. It was a fixed $8 \mathrm{GeV}$ kinetic energy storage ring, located in the MI tunnel directly above the MI beamline. The role of the Recycler ring was to allow storing a much larger current than that accepted by the Accumulator, and thereby increasing further the collider luminosity. When the maximum antiproton current was available in the Recycler, the $8 \mathrm{GeV}$ beam was transferred to the MI, accelerated to $150 \mathrm{GeV}$ and injected in the Tevatron, where a proton beam of the same energy circulating in the opposite direction had been previously stored.

\subsubsection{Tevatron Ring}

The Tevatron [31] was the last stage of the Tevatron accelerator chain. The Tevatron was a $1 \mathrm{~km}$ radius synchrotron able to accelerate the incoming $150 \mathrm{GeV}$ beams from MI to $980 \mathrm{GeV}$ (Tevatron Run II), providing a center of mass energy of $1.96 \mathrm{TeV}$. The proton and antiproton beams circulated in opposite directions in the same beam pipe. The beam was steered by 774 superconducting dipole magnets and focused by 240 quadrupole magnets with a maximum magnetic field of 4.2 Tesla. The employment of massive superconducting magnets, installed for the first time in an accelerator complex, required cryogenic cooling and consequently a large scale production and distribution of liquid helium. During Run II (after 2001) the Tevatron operated with 36 on 36 stored bunches. The antiprotons were injected after the protons had already been loaded. When the Tevatron loading was complete, the beams were accelerated to the maximum energy and data taking could start.

\subsubsection{Collider Performance}

The luminosity and the available center-of-mass energy are the two parameters that express the capability of colliding charged particle beams to produce new particles and quantify the performance of colliders. The centerof-mass energy defines the accessible phase space for the production of final state particles and at the Tevatron was $\sqrt{s}=1.96 \mathrm{TeV}$. Luminosity measures the instantaneous rate of beam-beam interactions at the collision points, 
normalized to the interaction cross section. An approximate expression for it in terms of the incoming beam intensities and sizes is given by:

$$
\mathcal{L} \approx \frac{N_{B} N_{p} N_{\bar{p}} f}{2 \pi\left(\sigma_{p}^{2}+\sigma_{\bar{p}}^{2}\right)}
$$

where $N_{B}$ is the number of bunches, $N_{p}$ and $N_{\bar{p}}$ are the number of proton and antiproton per bunch, $f$ is the bunch revolution frequency, $\sigma_{p}$ and $\sigma_{\bar{p}}$ are the effective widths of the beams. Making $\sigma_{p}$ and $\sigma_{\bar{p}}$ smaller and $N_{p}$ and $N_{\bar{p}}$ larger results in larger rate of collisions.

In the period between 2001 and 2011, several improvements were applied to Tevatron collider complex. The Tevatron performance history of this period (see Figure 2.2a), shows that the peak luminosity has grown from $\mathcal{L} \approx 8 \times 10^{30}$ $\mathrm{cm}^{-2} \mathrm{~s}^{-1}$ to $\mathcal{L} \approx 430 \times 10^{30} \mathrm{~cm}^{-2} \mathrm{~s}^{-1}$. The Tevatron program was terminated on September 30, 2011. The data collected by the experiments is proportional to the "integrated luminosity" during the runs, i.e. the time integral of the instantaneous luminosity, $L=\int \mathcal{L} d t$. The Tevatron integrated luminosity has greatly progressed over the years too (see Figure 2.2b). During the Run II the Tevatron delivered $12 \mathrm{fb}^{-1}$ of integrated luminosity per experiment, about $85 \%$ of which was collected by the CDF and $\mathrm{D} \emptyset$ detectors. The rate of the Tevatron luminosity progress was one of the fastest among high energy colliders. The continued Tevatron luminosity increase was mainly due to a larger number of antiprotons being available with the introduction of the electron cooling technique [62] in the Recycler, which in turn was the result of a continuous and dedicated effort of hundreds of experts to optimize and improve antiproton accumulation and cooling. The antiproton stochastic and electron cooling methods were not invented at Fermilab, but they were perfected to a degree not achieved anywhere else in the world. 


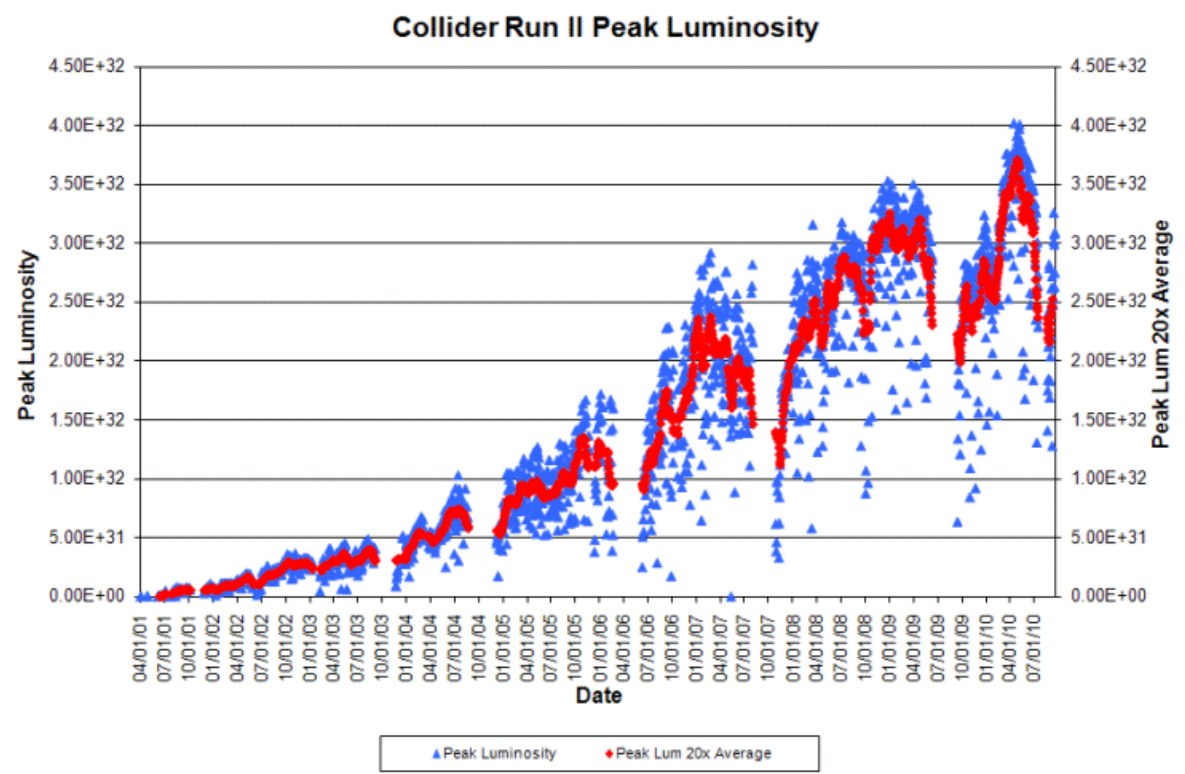

(a) Graphic of Tevatron peak luminosity since the start of RunII (2001-2011).

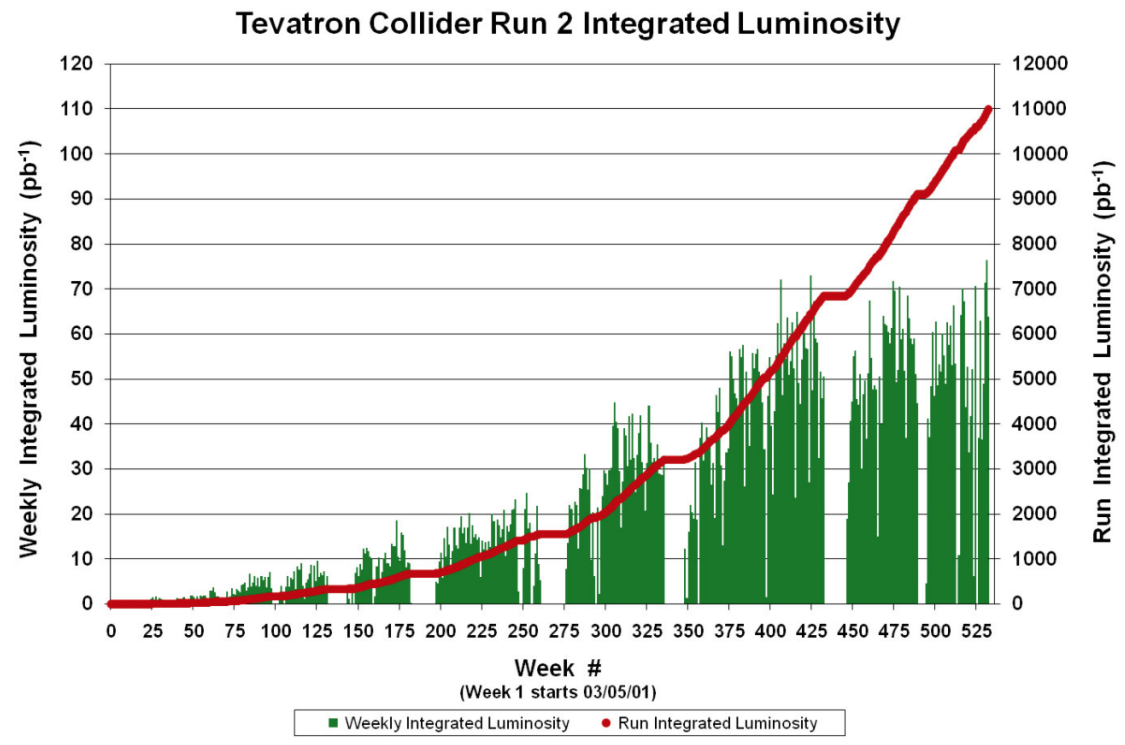

(b) Weekly and total integrated luminosity over Tevatron Run II.

Figure 2.2: Luminosity performance at Tevatron as a function of time. 


\subsection{The CDF II Detector}

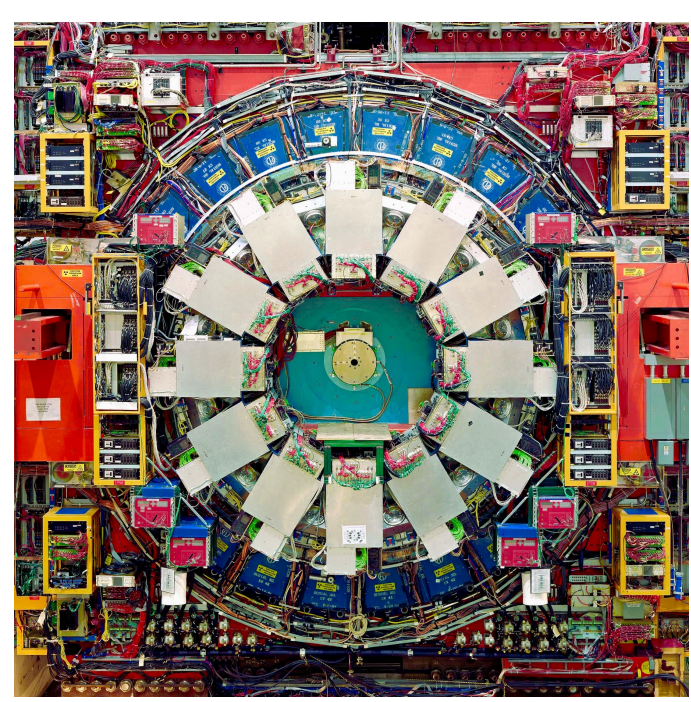

Figure 2.3: End view of the CDF II detector.

Experimental results rely heavily on accurate understanding of the detector. Two aspects are critical: the identification of objects that make up each signature, and the understanding of the calibration and resolution of the detector. The objects for which CDF has a good understanding of the efficiencies and fake-rates are those for which tracking is essential: electrons, muons, and photons (no track observed in the tracker), all in the central region. Similarly, the energy scale and resolutions of the calorimeters are well understood in the central region, where the magnetic spectrometer provides essential help in calibrating the calorimeters. The CDF II detector (ref $[10$ and figure 2.3), operating from 2001 to 2011, was a general-purpose particle detector with a cylindrical layout and azimuthal and forward-backward symmetry. An isometric view of the detector is shown in Figure 2.4. CDF II was composed of several specialized subsystems arranged in concentric layers, each one aimed at performing a specific task. The subsystems are listed below.

- The tracking system comprises three silicon microstrip trackers (Layer00, SVXII and ISL) and an open-cell drift chamber (COT) inside a superconducting solenoid, that provides a constant $1.4 \mathrm{~T}$ magnetic field parallel to the beam direction, with the purpose of bending into helices the trajectories of charged particles to allow the determination of their momentum and charge.

- The Time of Flight system (TOF), located inside the magnet solenoid just outside the COT, for measuring the time of flight of long-living particles and for determining the mass of charged particles with momenta up to $2 \mathrm{GeV}$.

- The calorimeter system, with the purpose of measuring the energy of charged and neutral showering particles. 


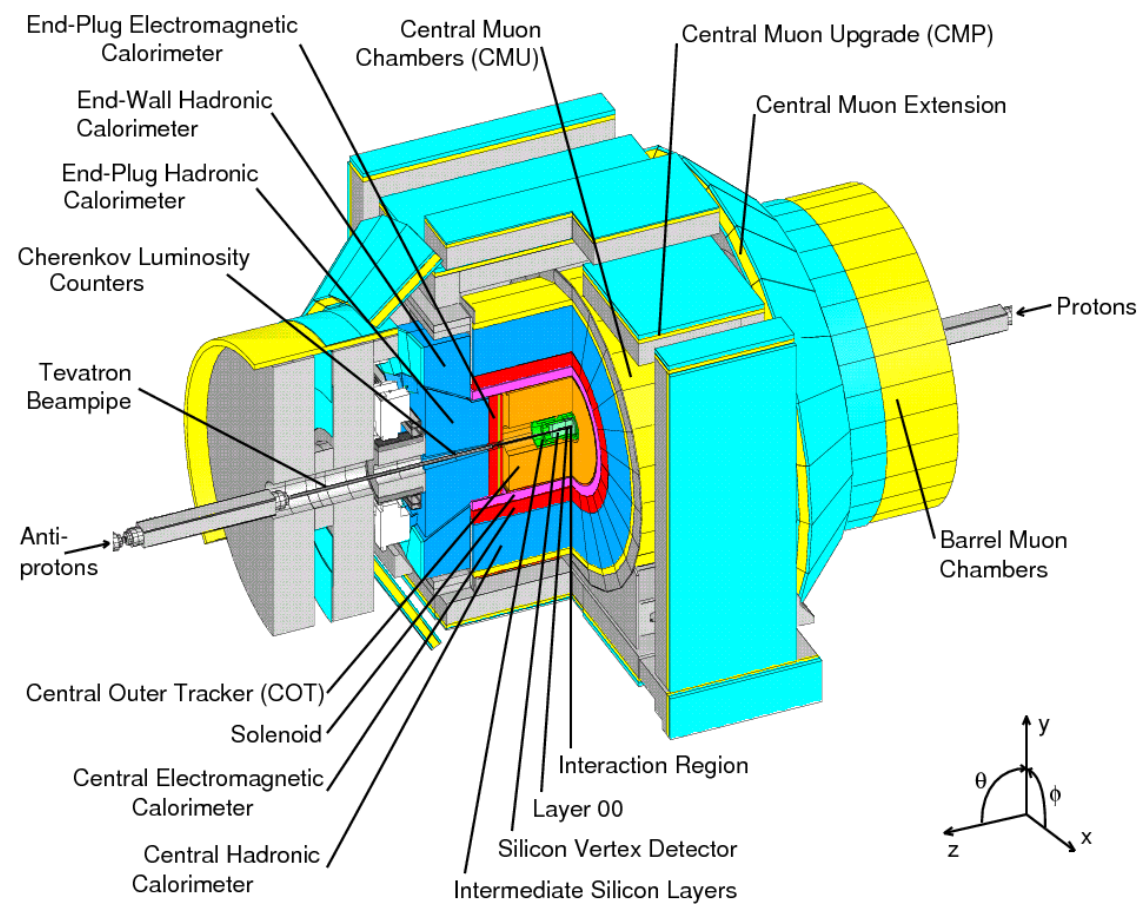

Figure 2.4: Isometric view of the CDF II detector. The main components are indicated.

- The muon chambers and scintillators, used to track and identify muons, that pass through the calorimeters interacting as minimumionizing-particles (m.i.p.).

- The luminosity monitors, for the instantaneous luminosity measurement, necessary to derive cross section from event yields.

\subsubsection{The Detector Coordinate System}

CDF II employs a right-handed Cartesian coordinate system with origin at the $\mathrm{B} \emptyset$ interaction point, assumed coincident with the center of the drift chamber. The positive $z$-axis lies along the nominal beam-line pointing toward the proton direction (east). The $(x, y)$ plane is perpendicular to either beams, with positive $y$-axis pointing vertically upward and positive $x$-axis in the horizontal plane of the Tevatron, pointing radially outward with respect to the center of the ring. Longitudinal refers to components along the $z$-axis, and transverse refers to components perpendicular to the $z$-axis. Since the colliding beams of the Tevatron are unpolarized, the resulting physical observations are invariant under rotations around the beam line axis. Thus, 
a cylindrical $(r, \phi, z)$ coordinate system is particularly convenient to describe the detector geometry, where

$$
r=\sqrt{x^{2}+y^{2}} \text { and } \phi=\tan ^{-1} \frac{y}{x}
$$

where $\phi=0$ is chosen in the $x$ direction. In the Tevatron collisions the longitudinal momentum of the colliding partons is unknown on an event-byevent basis. To describe the direction of a produced particle it is customary to use a variable invariant under $\hat{z}$ boosts as a unit of relativistic phase space, instead of the polar angle $\theta$. This variable is the rapidity defined as

$$
Y=\frac{1}{2} \ln \left[\frac{E+p \cos \theta}{E-p \cos \theta}\right],
$$

where $(E, \vec{p})$ is the energy-momentum four-vector of the particle. Under a $\hat{z}$ boost to an inertial frame with velocity $\beta$, the rapidity of a particle transforms linearly, according to $Y \rightarrow Y^{\prime} \equiv Y+\tanh \beta^{-1}$, therefore $Y$ is invariant since $d Y=d Y^{\prime}$. However, a measurement of rapidity still requires a detector with accurate particle identification capabilities because of the mass term entering $E$. Thus, for practical reasons, in the approximation that the mass of the particle is negligible, it is preferred to substitute $Y$ with the pseudorapidity $\eta$, which converges to the definition of rapidity in the ultra-relativistic limit and is defined as:

$$
\eta=-\ln \left[\tan \frac{\theta}{2}\right] .
$$

Other convenient variables used are the transverse component of the momentum $p_{T}=p \sin \theta$, the transverse energy, $E_{T}=E \sin \theta$, and the angular distance $\Delta R$, invariant under longitudinal boosts, defined as

$$
\Delta R=\sqrt{\Delta \phi^{2}+\Delta \eta^{2}} .
$$

$\Delta R$ defines a cone around a single particle and it is a very important parameter in the jet reconstruction algorithm.

\subsubsection{The Tracking System}

Three-dimensional charged particle tracking is achieved through an integrated system consisting of three silicon inner sub-detectors and a large outer drift-chamber, all contained in a superconducting solenoid, producing 1.41 T magnetic field. A schematic view of the tracking systems is illustrated in Figure 2.5. 


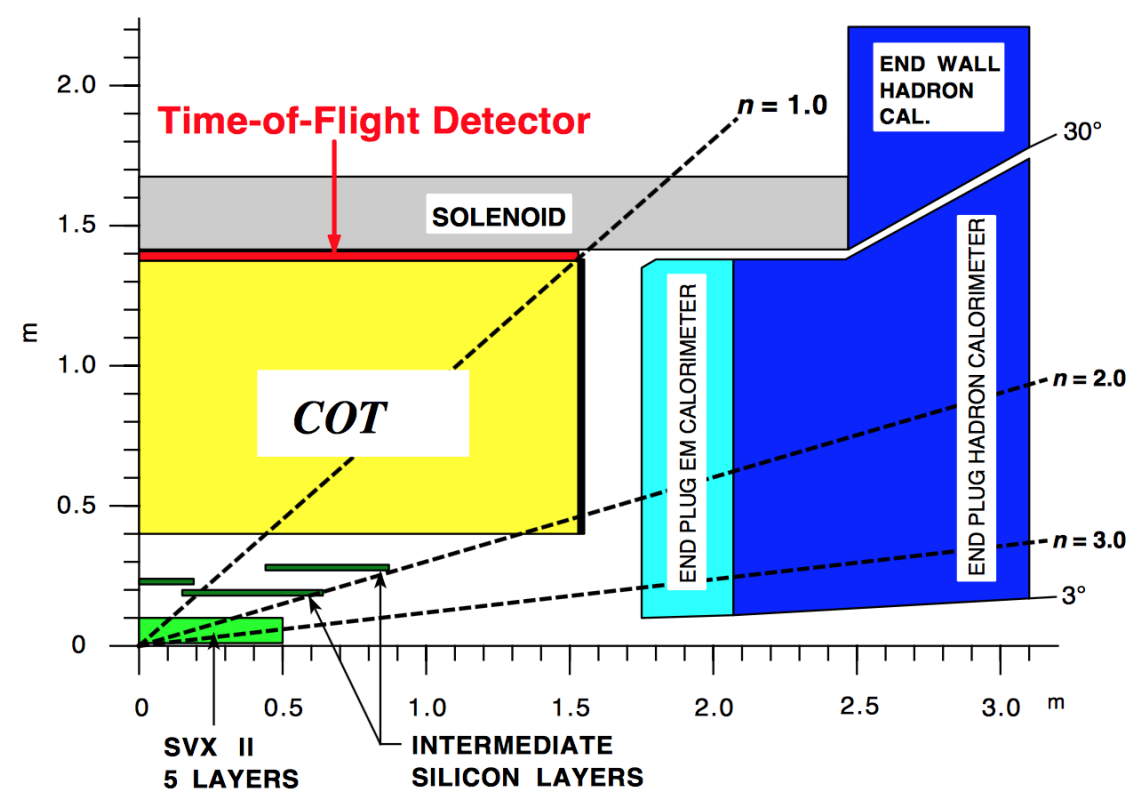

Figure 2.5: The CDF II tracker layout showing the different sub-detector systems.

The silicon detectors provide a precise determination of the track impact parameter, the azimuthal angle and the $\mathrm{z}$ coordinate at production, whereas the drift chamber has excellent resolution on the transverse momentum, $\phi$ and $\eta$. The combined information of the tracking detectors provides very accurate measurements of the helical paths of charged particles inside the detector.

The Silicon Tracker [53] is composed of three main parts. The core of the silicon detector is the Silicon VerteX detector SVXII. It is a fine resolution silicon micro-strip vertex detector which provides five three-dimensional samplings of tracks with full pseudorapidity coverage in the $|\eta| \leq 2$ region. The SVXII has a cylindrical geometry coaxial with the beam, and its mechanical layout is segmented in three $32 \mathrm{~cm}$ axial sections (barrels) $\times$ twelve $30^{\circ}$ azimuthal sectors $($ wedges $) \times$ five equally-spaced radial layers. A small overlap between the edges of adjacent azimuthal sectors helps wedge-towedge alignment. Sensors in a single layer are arranged into independent longitudinal read-out units, called ladders. A pictorial view of the innermost silicon trackers is illustrated in Figure 2.6. This detector provides position information with a $12 \mu \mathrm{m}$ resolution, allowing more determination of the trajectories and identification of decay-vertices displaced from the beam-line. The SVXII has an outer and an inner extension. 


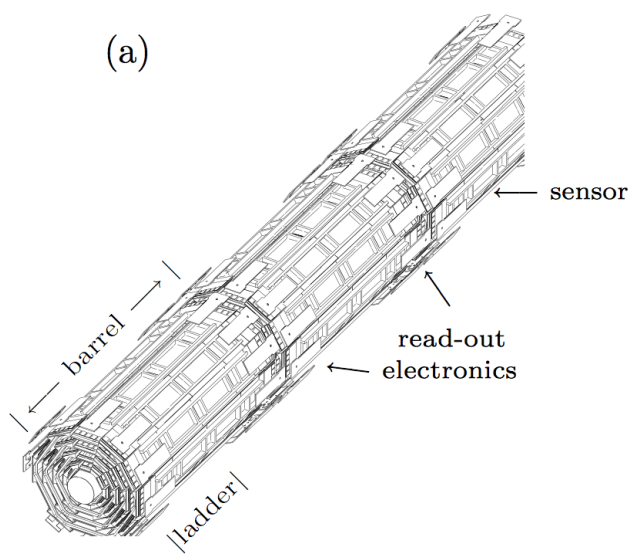

(b)

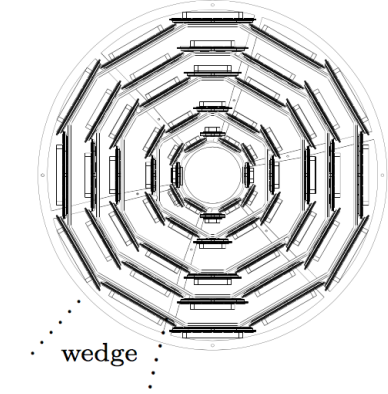

Figure 2.6: Schematic illustration of the three instrumented mechanical barrels of SVXII (a) and of the cross-section of a SVXII barrel in the $(\mathrm{r}, \phi)$ plane (b).

The outer extension, i.e., the Intermediate Silicon Layers (ISL), is a silicon tracker providing a single (double at forward angles) three-dimensional measurement in the central region, at intermediate radial distance from the drift chamber. The ISL allows efficient linking between tracks reconstructed in the drift chamber and hits detected in the SVXII, and extends the track finding at pseudorapidities $1 \leq|\eta| \leq 1$, where the chamber coverage is limited.

The inner extension, i.e., the Layer00 (L00), is a light-weight silicon layer placed on the beam-pipe. It recovers the degradation in resolution of the reconstructed vertex position due to multiple scattering on the SVXII read-out electronics and cooling system, installed within the tracking volume. Being so close to the beam, L00 allows to reach a resolution of $\approx 25-30 \mu \mathrm{m}$ on the impact parameter of tracks of moderate $p_{T}$, providing a powerful tool to identify long-lived hadrons containing a b quark.

The Central Outer Tracker (COT) $[54$ is a multi-wire, open-cell drift chamber, lying outside the silicon detector, which provides charged particle tracking up to $r=137 \mathrm{~cm}$ in the central pseudorapidity region $|\eta| \leq 1$. The COT contains 96 sense wire layers that are grouped in eight superlayers, as inferred from the end plate section shown in Figure 2.7. Each superlayer is divided in $\phi$ into supercells, and each supercell has 12 sense wires and a maximum drift distance that is approximately the same for all superlayers. Therefore, the number of supercells in a given superlayer scales approximately with the radius of the superlayer. The entire COT contains 30240 sense wires. Approximately half the wires run along the $z$ direction (axial wires), the other 
half are strung with a small (2 degrees) stereo angles with respect to the $z$ direction (stereo wires). The combination of the axial and stereo information allows to measure the $z$ positions and a three- dimensional reconstruction of tracks.

The COT is filled with an argon-ethane mixture (50:50); the drift time of ionization electrons produced by particles crossing the chamber is $<100 \mathrm{~ns}$. The central tracker provides a resolution on the track transverse momentum of $\sigma_{p_{T}} / p_{T}^{2} \simeq 0.01[\mathrm{GeV}]^{-1}$. The hit position resolution in the $r-\phi$ plane is about $140 \mu \mathrm{m}$. Tracking algorithms are used to reconstruct particle trajectories (helices) that best fit to the observed hits. The reconstructed trajectories are referred to as tracks. Particle momentum and charge are determined from the bending of tracks in the magnetic field.

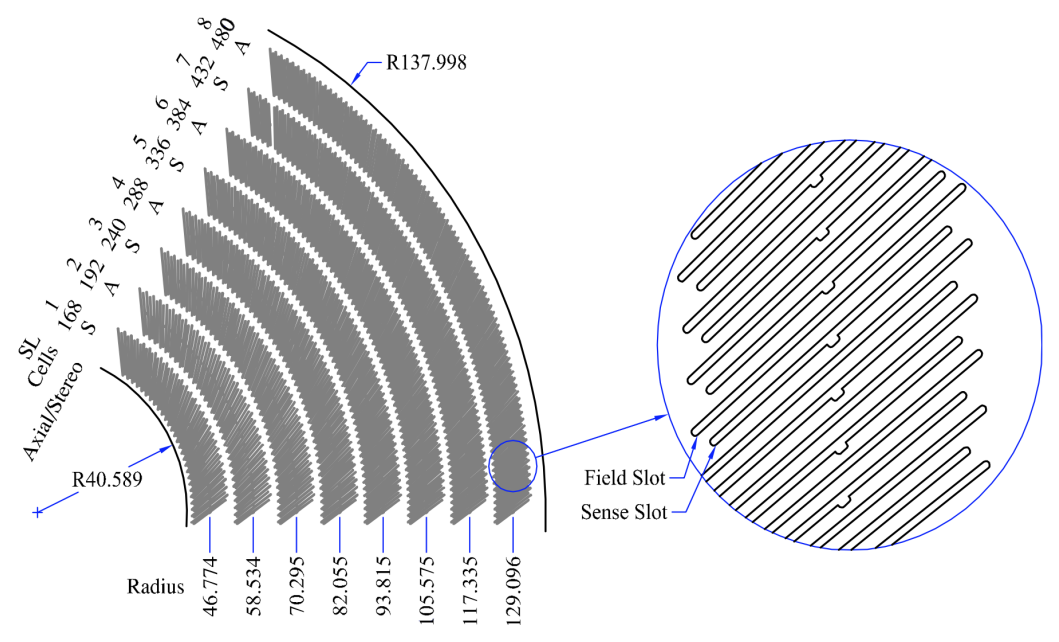

Figure 2.7: Section of the COT end plate. For each superlayer is given the total number of supercells, the wire orientation (axial or stereo), and the average radius. The enlargement shows the sense and field slot geometry in detail. Dimensions are in $\mathrm{cm}$.

\subsubsection{The Time of Flight system}

The CDF II capability of identifying charged hadrons is expanded at low momenta by the Time of Flight detector (TOF) [51]. By measuring the arrival time $(t)$ of a charged particle with respect to the bunch-crossing time, the TOF infers the mass of the particle according to the relation $m=\frac{p}{c} \sqrt{\frac{c^{2} t^{2}}{L^{2}}-1}$, where the momentum $p$ and the path length $L$ are precisely measured by the tracking system. A cylindrical array of 216 scintillating bars, oriented 
along the beam axis, is installed between the outer surface of the COT and the cryostat of the superconducting solenoid. Long attenuation-length and fast rise-time scintillator, along with accurate calibrations, ensure a measured time resolution of $\sigma_{t} \approx 110 \mathrm{ps}$.

\subsubsection{Calorimeters}

The CDF calorimetric system has been designed to measure energy and approximate direction of neutral and charged particles leaving the tracking region. In particular, it is devoted to jet reconstruction and it is also used to measure the missing energy associated to neutrinos. Particles hitting the calorimeter can be divided into two classes according to their interaction with matter: electromagnetically interacting particles, such as electrons and photons, and hadronically interacting particles, such as mesons or baryons produced in hadronization processes. To detect these two classes of particles, two different calorimetric parts were employed: the inner electromagnetic and the outer hadronic section. The calorimetric subsystems provide pseudorapidity coverage up to $|\eta| \leq 3.6$, and are segmented in $\eta-\phi$ sectors, called towers, projected towards the geometrical center of the detector. Each tower consists of alternating layers of passive material and scintillator tiles. The signal is read out via wavelength shifters (WLS) integrated in the scintillator and the light from WLS is carried through light guides to photomultiplier tubes. The energy $E$ measured in a given tower is the sum of the energy deposited in the electromagnetic calorimeter and in the hadronic calorimeter corresponding to that tower, $E=E_{E M}+E_{H A D}$. The depth of the calorimeter is measured in radiation lengths $X_{0}$ for the electromagnetic calorimeters [32]. For the hadronic calorimeters, the depth is measured in interaction lengths $\lambda$ [32]. The calorimetric system is subdivided into three regions, central, wall and plug, in order of increasing pseudorapidity, with the following naming convention: Central Electromagnetic (CEM), Central Hadronic (CHA), Wall Hadronic (WHA), Plug Electromagnetic (PEM) and Plug Hadronic (PHA). A quadrant view of the calorimetry system is shown in Figure 2.8.

CEM It is segmented in $\eta \times \phi=0.11 \times 15^{\circ}$ projective towers, called wedges, consisting of alternate layers of lead and scintillator, while the CHA and WHA, whose geometric tower segmentation matches the CEM one, use iron layers as radiators. Two position detectors are embedded in each wedge of CEM: a proportional chamber named CPR (Central Pre-Radiator) located just in front of the CEM (from the interaction point), and a Proportional strip detector (CES) located at the expected shower maximum $7 X_{0}$ into the tower. The interaction of particles with the solenoid and tracking material before 


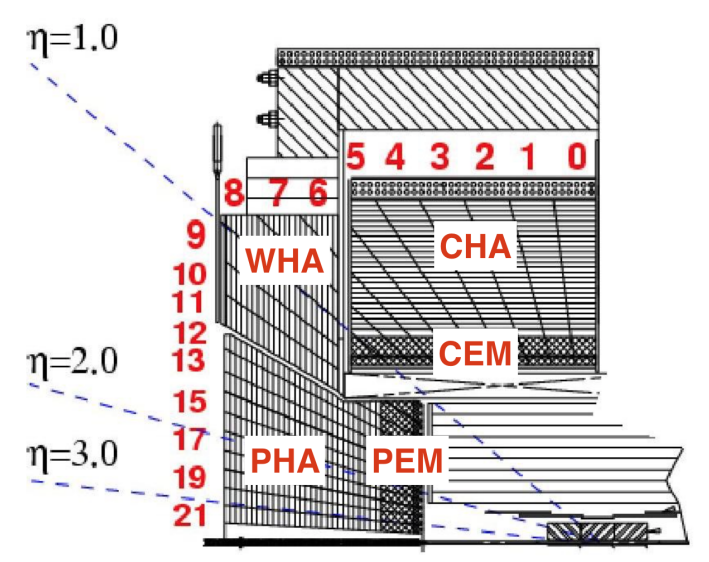

Figure 2.8: Quadrant view of the $\mathrm{CDF}$ calorimeter showing the electromagnetic (CEM, PEM) and hadronic compartments (CHA, WHA, PHA).

entering the calorimeter results in soft shower profiles that are measured in the CPR. The CES is a two-dimensional shower profile detector made of wires running along the $z$ direction and strips orthogonal to them. It provides valuable information used in the identification of electrons and photons, as illustrated in section 3.1.

The energy resolution of each segment of the calorimeter for a single particle has been measured using a test beam of electrons and can be parametrized as:

$$
\frac{\sigma_{E_{T}}}{E_{T}}=\frac{a}{\sqrt{E_{T}}}+b
$$

where the first term $a$ comes from sampling fluctuations and photostatistics of PMTs and the constant term $b$ comes from the residual errors in the intercalibration between the different towers and the non-uniform response of the calorimeter. For the CEM [47], the energy resolution parameters of high-energy electrons and photons are $a=14 \%[\mathrm{GeV}]^{1 / 2}$ and $b=2 \%$. Charged pions were used to evaluate the energy resolution in the hadronic calorimeters. Approximate resolution parameters for the CHA are $a=50 \%[\mathrm{GeV}]^{1 / 2}$ and $b=3 \%$, and for the WHA $a=75 \%[\mathrm{GeV}]^{1 / 2}$ and $b=4 \%[50$.

The Plug Calorimeter The plug calorimeter covers the pseudorapidity region $1.1 \leq|\eta| \leq 3.6$. Both electromagnetic and hadronic sectors are divided in 12 concentric regions each segmented in 48 or 24 (for $\eta<2.1$ or $\eta>2.1$ respectively) projective towers. As in the central calorimeter, 
there is the PEM and the PHA. Projective towers consist of alternating layers of absorbing material (lead and iron for electromagnetic and hadronic sectors respectively) and scintillator tiles. Resolution parameters for the plug calorimeter, measured on a test beam, are $a=16 \%[\mathrm{GeV}]^{1 / 2}, b=1 \%$ for the PEM [49], and $a=80 \%[\mathrm{GeV}]^{1 / 2}, b=5 \%$ for the PHA [39].

\subsubsection{The Muon Detector}

The CDF muon detector system [40] (Figure 2.4) consists of drift chambers and scintillator counters, and covers a pseudorapidity range up to $|\eta| \leq 1.5$. The muon subsystems are located in the outer part of the CDF II detector. This detector is organized in four subdetectors:

CMU The central muon detector (CMU) is located right outside the CHA behind $\sim 5$ nuclear interaction absorption lengths $\lambda_{0}$ of detector material. It covers the pseudorapidity range $|\eta| \leq 0.68$. It is composed of a barrel with inner and outer radii $r_{i}=347 \mathrm{~cm}$ and $r_{o}=396 \mathrm{~cm}$ respectively, containing 4 drift tube layers sectioned by wedge matching the CHA towers. Each tube operates in proportional mode, with a maximum drift time of $0.8 \mu \mathrm{s}$. The transtube multiple scattering resolution is $12 /(\mathrm{p}[\mathrm{GeV}]) \mathrm{cm}$ and the longitudinal resolution is $\delta_{z} \simeq 10 \mathrm{~cm}$.

CMP The central muon upgrade detector (CMP) is located outside the CMU behind $\sim 8 \lambda_{0}$ of detector material that includes additional $60 \mathrm{~cm}$ thick steel slabs. The CMP contains four layers of rectangularly arrayed drift tubes. The rapidity extension of the CMP detector is the same of CMU. The muons detected in this region are required to record hits in both CMU and CMP in order to improve the signal-to-background ratio. These muons are thus called CMUP muons. The CMP gas operation mode is proportional, the maximum drift time is $1.4 \mu \mathrm{s}$ and it has a transtube multiple scattering resolution of $15 /(\mathrm{p}[\mathrm{GeV}]) \mathrm{cm}$. A layer of scintillators mounted onto the outside surface of the CMP provides timing information with a resolution of 1-2 ns.

CMX The central muon extension detector (CMX) consists of conical sections facing toward the interaction point behind 6-9 $\lambda_{0}$ of detector material. The CMX system extends the central muon detector pseudorapidity coverage in the region $0.65 \leq|\eta| \leq 1.0$. The CMX detector contains two folds of 4 layers of rectangular drift tubes. The transtube multiple scattering resolution is $13 /(\mathrm{p}[\mathrm{GeV}]) \mathrm{cm}$ and the longitudinal position resolution is $\delta_{z} \simeq 14 \mathrm{~cm}$. Two layers of scintillators provides timing information. 
IMU The intermediate muon detector (IMU) is shielded by iron toroids at small angles, behind 6.2-20 $\lambda_{0}$ of material, depending on the rapidity. It consists of two barrels which extends the CDF geometric muon acceptance in the pseudorapidity range $1.0 \leq|\eta| \leq 1.5$. It contains four layers of proportional drift tubes, with a maximum drift time of $0.8 \mu \mathrm{s}$. The transtube multiple scattering resolution is $13-25 /(\mathrm{p}[\mathrm{GeV}]) \mathrm{cm}$ and the longitudinal position resolution is $\delta_{z} \simeq 16.5 \mathrm{~cm}$. Three layers of scintillators are mounted to provide timing information. The $\eta, \phi$ coverage of the muon detectors is shown in Figure 2.9.

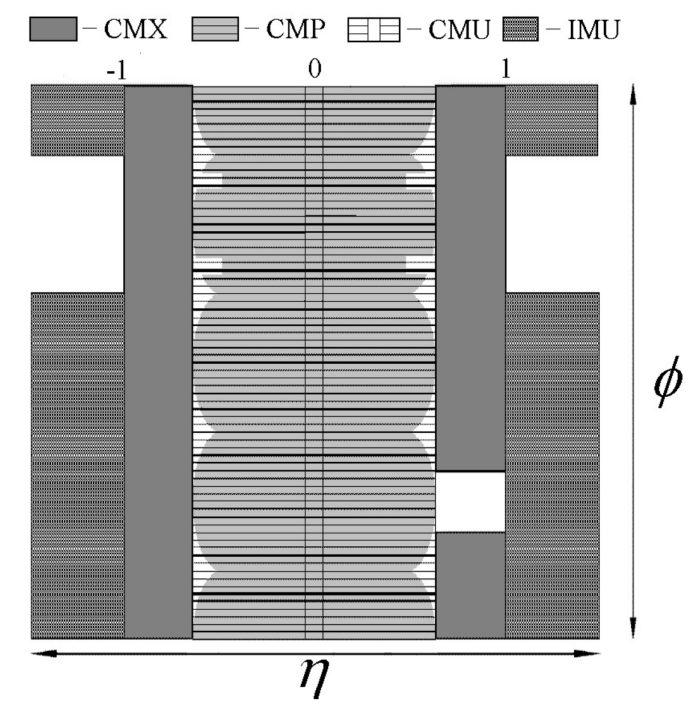

Figure 2.9: Coverage in $\eta$ and $\phi$ of the muon detector system. The shape is not regular because of the obstruction by structural elements.

\subsubsection{Cherenkov Luminosity Counters}

CDF measures the collider luminosity with a coincidence between two arrays of Cherenkov counters, the CLC [35], placed around the beam pipes on the two detector sides. They are located inside the endplug calorimeters, in the forward and backward regions $3.7 \leq|\eta| \leq 4.7$. Cherenkov counters are arranged around the beam pipe in three concentric cones with 16 counters each and pointing to the center of the interaction region. The counters measure the average number of interactions per bunch crossing $\mu$, which is used to provide a measurement of the instantaneous luminosity $\mathcal{L}$ :

$$
\mu \cdot f=\sigma_{p \bar{p}} \cdot \mathcal{L}
$$


Here, $\sigma_{p \bar{p}}$ is the fraction of the total $p \bar{p}$ inelastic cross section accepted by the monitors, as computed in dedicated MC simulations, and $f$ is the bunch crossing rate of the Tevatron. The $p \bar{p}$ accepted inelastic cross section at $\sqrt{s}=1.96 \mathrm{TeV}$ is $\sigma_{p \bar{p}} \approx 60 \mathrm{mb}|35|$. This value has been extrapolated from the measurement at $\sqrt{s}=1.8 \mathrm{TeV}$. The integrated luminosity is measured with a systematic uncertainty of $5.8 \%$.

\subsubsection{CDF Trigger System}

At hadron colliders the collision rate is much higher than the rate at which data can be stored on tape. Tevatron bunches were separated by 396 ns, leading to a crossing rate of $2.5 \mathrm{MHz}$, while the maximum tape writing speed was $\sim 100$ events per second. The role of the trigger is to efficiently select the most interesting physics events. Events selected by the trigger system are saved permanently on a mass storage and subsequently reconstructed offline. The CDF trigger system had a three-level architecture, each level provides a rate reduction sufficient to allow processing in the next level with minimal dead time. Figure 2.10 shows a schematic representation of the CDF data acquisition system. The final decision taken by each level is based on a set of programmable conditions that increase in complexity and selectivity until eventually data elaboration becomes possible.

Level 1 (L1) is constituted by a specialized hardware, providing a raw reconstruction of physics objects. The L1 system is organized in three parallel streams that take prompt information from different detectors and feed them to the Global Level 1 decision unit. The first stream uses the information provided by the eXtremely Fast Tracker (XFT), a powerful hardwired algorithm designed to feed the COT tracking information to the L1 system. The returned tracks are extrapolated to the calorimeter wedges and to the muon chambers. The second stream takes information from the calorimetric system. The third stream collects the information coming from the muon chambers, matching it with the XFT tracks.

At Level 1 the rate of accepted data is strongly reduced: about $97 \%$ of the events are rejected reducing the $2.53 \mathrm{MHz}$ rate to $<50 \mathrm{KHz}$.

Level 2 (L2) uses the partial reconstruction of physics objects to make a decision, exploiting the data collected from L1 and from electromagnetic calorimeter. It is organized in two streams: the first one is based on a hardware processor which collects information from calorimeters while the second, simultaneously, is based on a processor that find tracks in the silicon 


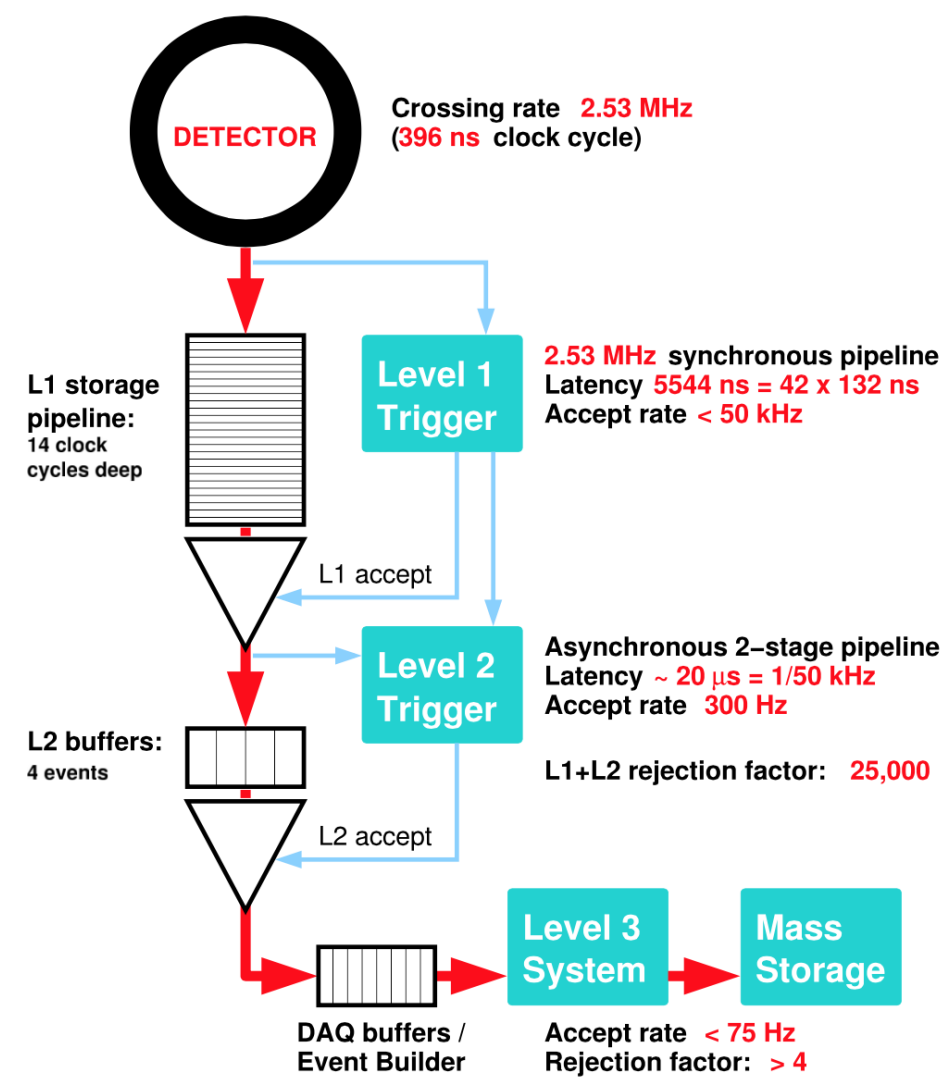

Figure 2.10: Schematic representation of the CDF II trigger and data acquisition systems.

vertex detector. The total latency of L2 is $21 \mu$ s while the output event rate input to L3 is $300 \mathrm{~Hz}$.

Level 3 (L3) is a software-based trigger that runs on a LINUX farm of commercial computers where the events are fully reconstructed using $\mathrm{C}++$ code. The information coming from L2 is addressed to the EVent Builder (EVB) which process the event using algorithms similar to the offline reconstruction. The final decision to accept an event is based on a series of features of interest for the physics process under study. Exit rate after L3 was variable, with an average rate of $50 \mathrm{~Hz}$ and a peak of $120 \mathrm{~Hz}$.

Top Events Triggers There are three different top event triggers used for collecting lepton + jets candidates. The CEM trigger selects high- $E_{T}$ electrons in CEM, the CMUP trigger selects high- $p_{T}$ muons in CMU and CMP, and 
the CMX trigger selects high- $p_{T}$ muons in CMX. Specifically, the CEM trigger requires, at $\mathrm{L} 1$, a COT track with $p_{T}>8 \mathrm{GeV}$ pointing to a CEM tower with $E_{T}>8 \mathrm{GeV}$ and a ratio of electromagnetic to hadronic energy $E_{\text {had }} / E_{\text {em }}<0.125$. At L2, the calorimeter clustering is performed, and the requirement is a cluster with $E_{T}>16 \mathrm{GeV}$ matched to a $p_{T}>8 \mathrm{GeV}$ track. Finally, at L3, the final reconstructed energy of the electron is required to pass $E_{T}>18 \mathrm{GeV}$ with a matching track of $p_{T}>9 \mathrm{GeV}$. The $E_{\text {had }} / E_{\text {em }}<0.125$ requirement is also enforced at L2 and L3. The CMUP trigger requires, at L1, a track with $p_{T}>4 \mathrm{GeV}$ matched to a stub in CMU and CMP hits consistent with the observed CMU hits. No additional requirements are made at L2; L3 requires a final reconstructed $\mathrm{COT}$ track of $p_{T}>18 \mathrm{GeV}$ matched to stubs in CMU and CMP. The CMX trigger operates very similarly, but requires a track of $p_{T}>8 \mathrm{GeV}$ at L1 matched to a CMX stub.

The top event triggers used for the lepton+jets channel are used for the dilepton candidates as well. In the full hadronic channel and in the MET + jets channel, jet and MET triggers are used, respectively.

The events accepted by the trigger are written on fast-access disks to allow a real-time data collection, grouped in run numbers. All the manipulations on the data are done off-line and are referred as data handling. An important phase is the so called production, where the physics objects such as leptons, vertices and jets are reconstructed using algorithms that use the most up-todate detector calibration and the best measured beamlines. The processed data are organized into data sets that are the basis for the physics analyses. Several run numbers are grouped in run periods, each one corresponding a data taking period with similar characteristics.

The CDF collaboration has defined a list of standard data-quality requirements a run has to satisfy to be considered "good"; those runs are collected in the so-called Good Run List (GRL). All CDF analysis must conform to it. Furthermore, many specific good run lists, dedicated to analyses based on different subdetectors status, have been developed by different groups. Which list is the most suitable for a certain analysis depends on the subdetectors it uses. 


\section{Chapter 3}

\section{Physics Object Reconstruction}

Our analysis studies of $t \bar{t}$ production characterized by a lepton + jets event topology composed by a charged lepton, either an electron or a muon or a tau, a neutrino and a variable number of jets, including jets coming from $b$ quarks that must be identified.

After an event has been accepted online, a full event reconstruction is performed offline. From raw data collected by the detector, high level objects (tracks, vertices, calorimetric clusters) are reconstructed and combined to provide information on the events final state.

\subsection{Electron Reconstruction}

Electrons are identified by requiring a track matched to an energy cluster in the calorimeter with an appropriate shower profile. Depending on which part of the calorimeter system the cluster belongs to, the electrons are divided in Central (CEM) and Plug (PEM) electrons. In our analysis we only use CEM electrons which are reconstructed from electromagnetic clusters in the central calorimeter, covering a region in pseudorapidity up to $|\eta| \leq 1.0$. Additional selection criteria applied to identify CEM electrons are listed below and summarized in Table 3.1. The particle 4-momentum is reconstructed assuming massless electrons.

- To be accepted as a tight CEM electron, the electron must have $|\eta| \leq 1.0$ and $E_{T}>20 \mathrm{GeV}$ matched to a COT track passing various track quality cuts.

- To further reject showers from hadrons, the primary simple electron selection cut applied to the ratio between the energy deposited in the hadronic calorimeter and the energy deposit in the electromagnetic one 
is tightened to $E_{h a d} / E_{e m}<0.055+0.00045 \cdot E_{T} / \mathrm{GeV}$. The additional linear term accounts for the fact that higher-energy electrons will leak more energy into the hadronic calorimeter.

- The ratio of the calorimeter energy to the COT track momentum, $E / p$ $\left(=E_{T} / p_{T}\right)$, is required to be at most 2.0 if $E_{T}<100 \mathrm{GeV}$. (Above 100 $\mathrm{GeV}$ this cut becomes unreliable and is not used.)

- $L_{s h r}$, a variable describing the match of the lateral profile shape to the expected shape for electrons derived from test-beam data, must be $<$ 0.2 .

- A $\chi^{2}$ comparison is used to compare the shape of the profile measured in the Central Electromagnetic Strip chamber (CES) to the expected profile and $\chi_{\text {stripes }}^{2}<10.0$ is required.

- The distances between the extrapolated COT track and the position of the CES cluster, $\Delta x$ in the $r-\phi$ plane and $\Delta z$ in the $r-z$ plane, are required to satisfy $-3.0 \mathrm{~cm}<Q \cdot \Delta x<1.5 \mathrm{~cm}$ and $|\Delta z|<3.0 \mathrm{~cm}$, where $Q$ is the sign of the charge.

- Electrons produced by photon conversions are rejected searching for a close track of opposite sign, $\Delta(x y)<2 \mathrm{~mm}$, and $\Delta \cot \theta<0.04$, where $\Delta(x y)$ is the distance in the $r-\phi$ plane at the point where the tracks are parallel. If such a track is found, the electron is assumed to come from a pair production and is rejected.

- To identify the electron as coming from a $W$ decay (as opposed to a decay of a heavy-flavor hadron), the electron is required to be isolated from other sources of energy in the calorimeter. This is enforced by measuring the additional energy in a cone of radius $\Delta R=0.4$ around the electron; the ratio of this additional energy to the electron energy must be less than 0.1 .

\subsection{Muon Reconstruction}

Muons pass through the entire CDF detector, in great majority with an energy release of a minimum ionizing particle (m.i.p.) in the calorimeters. They leave hits in the outer muon chambers. A muon is reconstructed starting from a track in the COT and adding track segments (stubs) formed with hits in the muon drift chambers. Many reconstruction categories exist, some of them 
Table 3.1: Selection requirements applied for CEM electron identification.

\begin{tabular}{lc}
\hline Parameter & Requirement \\
\hline$E_{T}$ & $>20 \mathrm{GeV}$ \\
$|\eta|$ & $<1.0$ \\
$E_{\text {had }} / E_{e m}$ & $<0.055+0.00045 \cdot \mathrm{E} / \mathrm{GeV}$ \\
$E / p$ & $<2.0$ if $E_{T}<100 \mathrm{GeV}$ \\
$L_{s h r}$ & $<0.2$ \\
$Q \cdot \Delta x$ & $>-3.0 \mathrm{~cm} \mathrm{and}<1.5 \mathrm{~cm}$ \\
$|\Delta z|$ & $<3.0$ \\
$\chi_{\text {strips }}^{2}$ & $<10.0$ \\
Conversions & Rejected \\
Isolation & $<0.1$ \\
\hline
\end{tabular}

require the muons to have hits in one muon detector subsystem. Since CMP covers the same $|\eta|$ range as CMU, muons in this region are required to record hits in both CMU and CMP in order to improve the signal-to-background ratio. These muons are thus called CMUP muons. Loose muons pass the general track quality and isolation cut but they don't produce additional drift chamber stubs. Muon categories utilized for our analysis are: CMX, CMUP and loose muons.

The cuts used to identify muons are listed below and summarized in Table 3.2.

- To be identified as a tight CMUP or CMX muon, $p_{T}>20 \mathrm{GeV}$ and $|\eta|<1.0$ cuts are required.

- The energy deposited in the calorimeter is required to be consistent with a m.i.p. . This is enforced by requiring $E_{\text {em }}<2 \mathrm{GeV}$ and $E_{\text {had }}<6$ $\mathrm{GeV}$ for muons having $p_{T}<100 \mathrm{GeV}$ and $E_{\text {em }}<2+\left(\frac{p_{T}}{\mathrm{GeV}}-100\right) \cdot 0.0115$ $\mathrm{GeV}$ and $E_{\text {had }}<6+\left(\frac{p_{T}}{\mathrm{GeV}}-100\right) \cdot 0.0280$ for muons with $p_{T}>100 \mathrm{GeV}$.

- The distance $|\Delta x|$ between the extrapolated COT track and the stub in the muon chambers is required to be $<3.0 \mathrm{~cm}$ in $\mathrm{CMU},<5.0 \mathrm{~cm}$ in $\mathrm{CMP}$, and $<6.0 \mathrm{~cm}$ in CMX.

- For CMP and CMX, the extrapolated tracks are also required to lie at least $3 \mathrm{~cm}$ away along the wire axis from the edges of the muon chambers to avoid chamber edge effects. 
- Muons originating from cosmic rays are identified and rejected by an algorithm which looks for tracks passing through the detector and rejected.

- Like electrons, an isolation cut of $<0.1$ is applied to the muon to require it to come from $W$ decay.

Table 3.2: Selection requirements applied for muon identification.

\begin{tabular}{lc}
\hline Parameter & Requirement \\
\hline$E_{T}$ & $>20 \mathrm{GeV}$ \\
$|\eta|$ & $<1.0$ \\
$E_{\text {em }}$ & $<2+\max (0,(p-100) \cdot 0.0115) \mathrm{GeV}$ \\
$E_{\text {had }} \mid$ & $6+\max (0,(p-100) \cdot 0.028) \mathrm{GeV}$ \\
$\left|\Delta x_{C M U}\right|$ & $<3.0 \mathrm{~cm}$ \\
$\left|\Delta x_{C M P}\right|$ & $<5.0 \mathrm{~cm}$ \\
$\left|\Delta x_{C M X}\right|$ & $<6.0 \mathrm{~cm}$ \\
Distance from z-axis edge & $>3.0 \mathrm{~cm}$ \\
Cosmic rays & Rejected \\
Isolation & $<0.1$ \\
\hline
\end{tabular}

\subsection{Jet Reconstruction}

Jets are frequently produced in the parton interactions at hadron colliders. They arise from the complex physical process of hadronization that evolves a color-charged parton into a collimated set of final state colorless hadrons, photons and leptons. By measuring the energy of a jet, we can thus get an estimate of the energy of the parton which originated it; this process involves a series of corrections to the measured ("raw") jet $E_{T}$. However, this introduces several significant sources of systematic uncertainties, which in turn results in a potentially large source of uncertainty in our final measurement.

Jets are identified using a jet clustering algorithm known as JetClu |37|; briefly, JetClu identifies seed towers in the calorimeter with an energy of at least $1 \mathrm{GeV}$ and adds groups of clusters in the electromagnetic and hadronic calorimeters within a cone of radius $\Delta R=0.4$ to the seed, where $\Delta R=$ $\sqrt{\Delta \eta^{2}+\Delta \phi^{2}}$. The centroid of the jet is then calculated by summing the $\eta$ and $\phi$ positions of the towers weighted by their $E_{T}$ and a new list of towers 
around the new center is calculated; this process is repeated iteratively until it converges. These measurements provide a resolution of approximately $\sigma\left(E_{T}\right) \approx 0.1 \cdot E_{T}+1.0 \mathrm{GeV}$.

The four-momentum assigned to a jet must be corrected to account for detector defects and reconstruction algorithm imperfections. In order to convert the measured transverse jet energy into the transverse energy of the partons, a set of corrections to the measured jet energy (raw energy) has been developed [34]. There are a total of five levels of corrections, numbered 1 through 7 (levels 2 and 3 correspond to corrections which are no longer used).

Pseudorapidity-dependent correction (L1) Level 1 accounts for nonuniformities in calorimeter response along $\eta$, primarily arising from the central crack at $\eta=0$ where the two halves of the calorimeter meet on a support structure. This correction is obtained by studying the $p_{T}$ balancing in dijet events, where one jet is required to be in the best region of the central calorimeter, $0.2<|\eta|<0.6$, and assuming that the $p_{T}$ of the two jets (events with additional hard radiation are rejected) should balance, as expected in a perfect detector.

Multiple interactions correction (L4) The number of interactions occurring during beam bunch crossings follows a Poisson distribution whose mean increases with instantaneous luminosity. L4 accounts for the different calorimeter occupancy as a function of the number of $p \bar{p}$ interactions occurring in the same bunch crossing. The number of interactions is measured by the number of vertices along the beamline, or z-vertices. This correction is obtained by taking random data ("minimum bias") and measuring the amount of energy in a randomly-selected cone in the central calorimeter region $0.2<|\eta|<0.6$ as a function of the number of observed z-vertices in the event.

Absolute energy scale corrections (L5) The absolute correction (L5) is designed to convert the measured jet energy into the energy of the particles inside the jet cone, accounting for the nonlinear response of the calorimeter. This correction is derived by using PYTHIA MC [70] dijet events and comparing particle jets at generator level (before they are passed through the detector simulation), with calorimeter jets, as obtained from the detector simulation.

Underlying event (L6) and out-of-cone (L7) corrections L6 and L7 are not actually used in defining the jet energies in our measurement, but we need to take into account the systematic uncertainties introduced by neglecting 


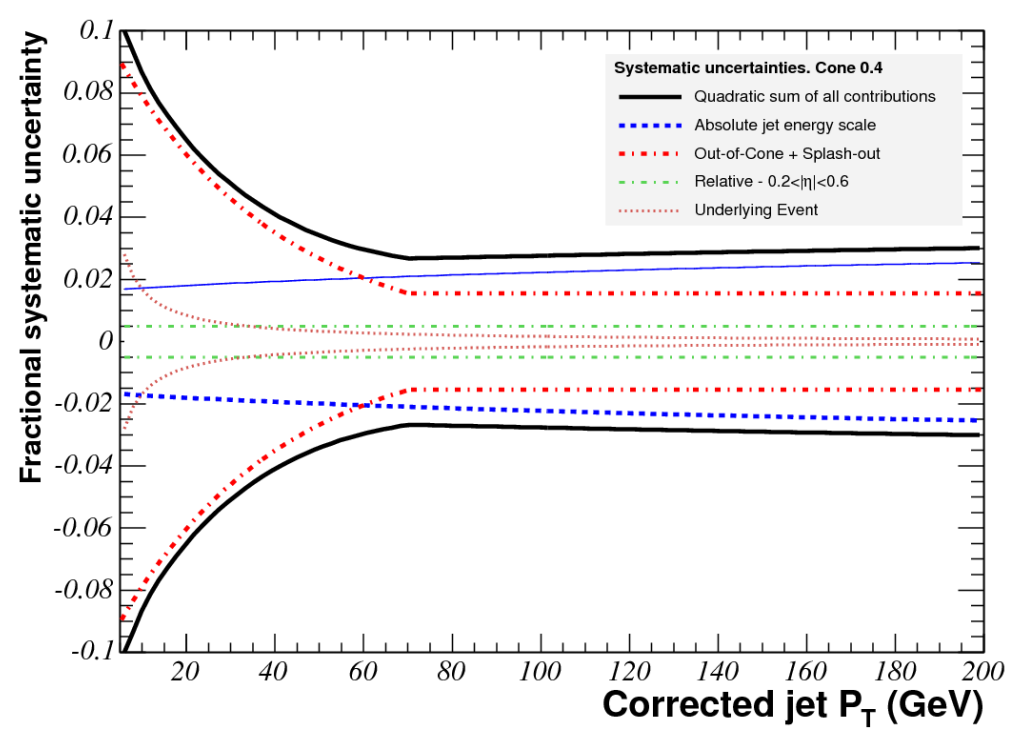

Figure 3.1: Systematic uncertainties on the measurement of jet energies as a function of jet $p_{T}$ for central jets.

these corrections. Reconstructed jet energies in hard $p \bar{p}$ interactions may contain contributions from particles created by interactions involving other partons in the colliding hadrons (spectator interactions) or by gluons from initial state radiation in the hard interaction. These contributions are called underlying event. On the other hand, a fraction of the parton energy may be lost outside the jet cone because of final state gluon radiation, fragmentation at large angles relative to the jet axis or low $p_{T}$ particles bending in the magnet field. L6 and L7 average contribution are obtained in simulations using PYTHIA and HERWIG [41] MC dijet samples.

The systematic uncertainties for these corrections are summarized in Figure 3.1. The total uncertainty is approximately $3 \%$ for high $-p_{T}$ jets and it can reach $10 \%$ for lower- $p_{T}$ jets; if taken directly as a systematic on our final measurement, this would be by far our largest single source of uncertainty. However, since we use events which contain a $W$ boson decaying to hadrons, we can use the known mass of the $W$ boson to obtain an additional constraint to the jet energy scale $(J E S)$ in our measurement. This technique is discussed further in section 5.6 . 


\subsection{Missing Transverse Energy Reconstruction}

Since for neutrinos the probability of interacting in the detector material is negligible, they escape detection causing an energy imbalance in the observed event. The transverse energy vector must be null in the final state as it is in the initial state of the events, and can serve to sense escaping neutrinos. The missing transverse energy is defined as follows:

$$
\overrightarrow{\mathbb{E}}_{T}=-\sum_{i} E_{T}^{i} \hat{n}_{i}
$$

where $E_{T}^{i}$ is the transverse energy measured in the $i$-th tower of the calorimeter and $\hat{n}_{i}$ is the projection of the versor pointing from the event vertex to the $i$-th calorimeter tower in the plane perpendicular to the beam axis. This raw measurement is corrected twice. As muons deposit a small fraction of their energy in the calorimeter, if isolated high- $p_{T}$ muons are found in the event the average ionization energy deposited by the muon in the calorimeter is subtracted and replaced by the muon $\vec{p}_{T}$. The $\mathbb{E}_{T}$ is also corrected to take into account the corrections applied to the $E_{T}$ of jets in the event.

\subsection{Secondary Vertex Identification}

Jets arising from $\mathrm{b}$ quark hadronization and decay are present in a wide range of physics processes, including top quark decays. The ability to accurately identify $b$ jets is fundamental in reducing the large background to identified $b$ jets from gluon jets, light-flavor quarks $(u, d, s)$ and from $c$ quark fragmentation. Characteristic features of $b$ quarks initiated jets are the relatively large mass, high track multiplicity, and high $p_{T}$ of decay products with respect to $b$-flight direction, and long lifetime of the heavy flavor hadrons. These features are used to develop algorithms that allow distinguishing $b$ jets from those produced by light-flavor quarks and gluons. In particular the B-hadrons, coming from the hadronization of $b$ quarks, have long natural lifetimes $(\sim 1.5 \mathrm{ps})$ and in top decays are sufficiently energetic to travel few mm before decaying $(c \tau \approx 450 \mu \mathrm{s})$. The tagging algorithms take advantage of this property and identify a point displaced from the primary vertex with tracks of large impact parameter $\left(d_{0}\right)$. The CDF silicon tracker described in section 2.2 .2 is primarily designed for this purpose. The algorithm employed at CDF is called Secondary Vertex Tagger (SECVTX). 
The SECVtx Algorithm [65] Tagging is performed for all jets with $|\eta|<$ 2.4 in an event. The algorithm searches for secondary vertices using the tracks within the jet cone. The usable tracks must satisfy the following requirements:

- $p_{T}>0.5 \mathrm{GeV}$;

- $\left|d_{0}\right|<0.15 \mathrm{~cm}$ and $\left|d_{0} / \sigma_{d_{0}}\right|>2.0$, where $d_{0}$ is the impact parameter relative to the beam axis and $\sigma_{d_{0}}$ its uncertainty;

- $\left|z_{0}-z_{P V}\right|<2.0 \mathrm{~cm}$, where $z_{0}$ represents the z-coordinate of the track and $z_{P V}$ the primary vertex coordinate;

- have more than a minimum number (depending on track reconstruction quality and position) of hits in the silicon detector;

- be confirmed in the COT.

If there are at least 2 tracks with the above requirements the jet is called taggable. Then, the algorithm tries to reconstruct a secondary vertex with at least three tracks of $p_{T}>0.5 \mathrm{GeV}$, of which one must have $p_{T}>1.0 \mathrm{GeV}$, or a vertex with at least two tracks of $p_{T}>1.0 \mathrm{GeV}$. If a secondary vertex is found the jets is tagged and a bi-dimensional decay length $L_{x y}$ is calculated as the projection in the $r-\phi$ plane of the SECVTX vector (going from the primary vertex to the secondary one) into the jet axis, as illustrated in Figure 3.2. A jet is considered tagged if $L_{x y}>7.5 \sigma_{L_{x y}}$, where $\sigma_{L_{x y}}$, the uncertainty on $L_{x y}$, is in average approximately $190 \mu \mathrm{m}$.

In order to measure the efficiency of the tagging algorithm for $t \bar{t}$ events, a two-step strategy is used. First, the tagging efficiency is measured in a sample enriched in heavy flavor; specifically, events with two back-to-back jets are selected, where one of the jets is required to contain a low- $p_{T}$ electron. We assume that the electron is produced by the decay of a heavy-flavor hadron, and so the other jet is also likely to originate from a heavy-flavor parton. Then, we can measure the tagging efficiencies in data and $\mathrm{MC}$. Because the MC does not model the secondary vertex tagging precisely, there is a scale factor between the data and $\mathrm{MC}$ efficiencies of approximately $0.92[65]$. The tagging efficiencies for jets in $t \bar{t}$ data can then be estimated by multiplying the tagging efficiencies obtained from $t \bar{t} \mathrm{MC}$ by this scale factor. 


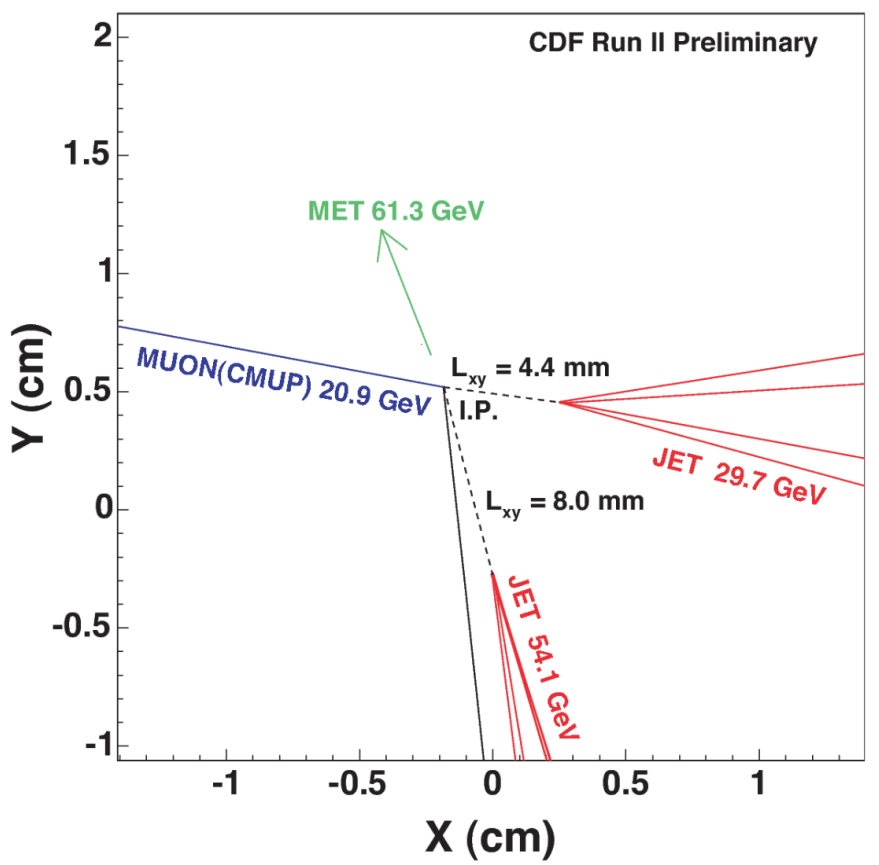

Figure 3.2: $W+$ jets candidate event with two secondary vertices tagged by SECVTX. The $\mathbb{E}_{T}$ direction, a muon track, a prompt track and tracks from the secondary vertices are shown. 


\section{Chapter 4}

\section{Event Selection}

\subsection{Lepton + jets topology}

As mentioned in section 1.2.2, the top quark decays almost exclusively into a $W$ boson and a $b$ quark. The final states of the $t \bar{t}$ system are determined by the independent decays of the two $W$ bosons produced in the $t$ and $\bar{t}$ decays. $W$ boson can decay either leptonically into any charged lepton-neutrino pair of the same family (with nearly family-independent branching ratio), or hadronically into a pair of the two lower mass quark doublets.

Even though the fully hadronic channel (where both bosons decay hadronically) offers a good branching ratio, it suffers from a large QCD background of multijet states. Measurement of the top properties in the dilepton channel (where both bosons decay leptonically) is complicated by the two nonobservable neutrinos in the final state. Also, even if the request of two leptons makes the signal over background ratio very large (even more so when the leptons have different flavor, $e-\mu$ events), this channel suffers from low statistics at the Tevatron! The single-lepton final states (usually named lepton + jets and characterized by one $W$ decaying leptonically and the other one decaying hadronically), involving either electrons or muons or tau leptons, can be fully reconstructed from the experimental observables and are best suited for measurements such as the top mass. Due to the presence of a lepton in the final state, QCD background affects less this channel if compared to the full hadronic one, still retaining a relatively large number of signal events.

For these reasons we perform our measurements selecting the lepton + jets final states where one $W$ decays leptonically into an electron $(e)$ or a muon

\footnotetext{
${ }^{1}$ The statistical limitation of the dilepton channel is not an issue at the LHC, where the top production cross section is two order of magnitude higher than the cross section at the Tevatron and the instantaneous luminosity is higher too.
} 
$(\mu)$ plus a neutrino $(\nu)$, including the cascade decay of $W \rightarrow \tau \nu$ and $\tau \rightarrow e \nu$ or $\tau \rightarrow \mu \nu$, and the other $W$ decays hadronically into a pair of jets.

Figure 4.1 shows a typical lepton + jets final state $t \bar{t} \rightarrow b(l \nu) \bar{b}\left(q \bar{q}^{\prime}\right)$.

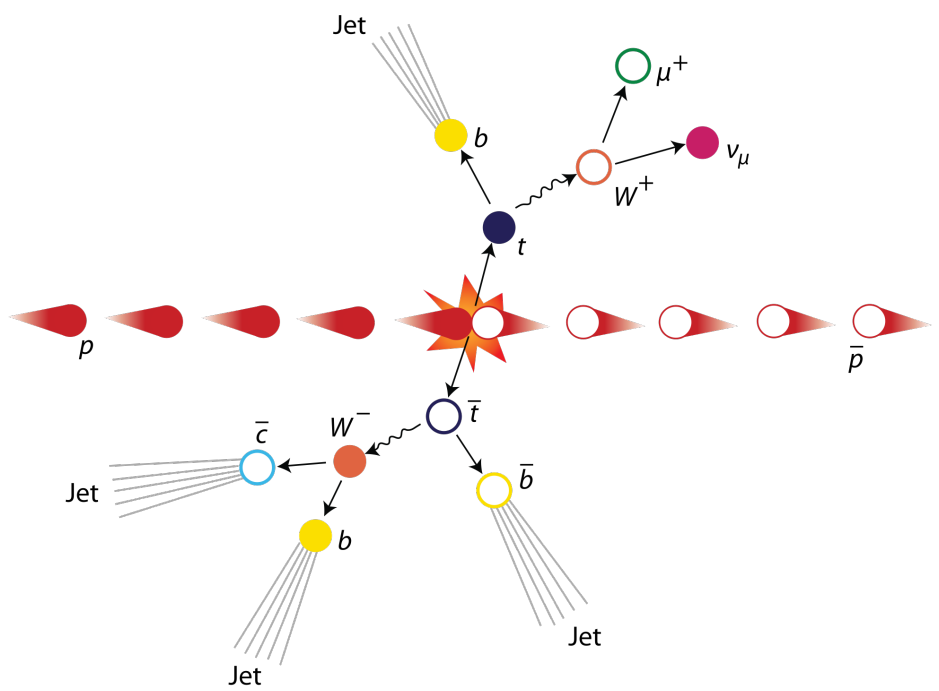

Figure 4.1: A typical lepton + jets event coming from the $t \bar{t}$ decay.

\subsection{Event Signature}

The signature of the lepton + jets events is constituted by a high $p_{T}$ charged lepton, large missing transverse energy $\mathbb{E}_{T}$ and at least four jets.

In particular, we select either one isolated CEM electron with $E_{T}>20$ GeV and pseudorapidity $|\eta| \leq 1.0$ or one isolated muon with $p_{T}>20 \mathrm{GeV}$ and $|\eta| \leq 0.68$ in the case of muon observed in CMUP detector, or $0.65 \leq|\eta| \leq 1.0$, in the case of muon detected in CMX. We also include loose muons, those which pass the general track quality and isolation cut but don't produce additional stub in the outer muon chambers. We recall here that a lepton is considered isolated when $\left(p_{T}^{\text {tot }}-p_{T}^{l}\right) / p_{T}^{l}<0.1$, where $p_{T}^{\text {tot }}$ is the total transverse momentum (energy) and $p_{T}^{l}$ is the lepton transverse momentum (energy) for muon (electron) in a cone of radius $\Delta R=0.4$ with axis along the direction of the lepton.

We also require the presence of large missing transverse energy, accounting for the escaping neutrino coming from the leptonic $W$ decay: $\mathbb{E}_{T}>20 \mathrm{GeV}$.

Jets are reconstructed by using the JetClu cone algorithm [37] with a cone of $\Delta R=0.4$. Jets are required to have $E_{T}>20 \mathrm{GeV}$ and $|\eta| \leq 2.0$, defining the tight jets, or $E_{T}>12 \mathrm{GeV}$ and $|\eta| \leq 2.4$, defining loose jets. Jets 
originating from $b$ quarks are identified (tagged) using the SECVTX algorithm [65. All jet variables are scaled by a factor which accounts for level 5 jet corrections, as described in chapter 3 .

We divide the sample of candidate lepton + jets events into sub-samples based on the number of identified $b$ jets, no $b$-tagged jet (0-tag), one $b$-tagged jet (1-tag), and two or more $b$-tagged jets (2-tag). In the 0 -tag events, we require exactly four tight jets. In the 1-tag and 2-tag events, three tight jets and at least one additional jet (tight or loose) are required. We divide the 1-tag and 2-tag samples into sub-samples based on the number of tight jets and call the "tight" sub-sample the one requiring exactly four tight jets and "loose" the one consisting of the remaining events passing selection (events with 3 tight jets and at least one loose jet and events with more than four tight jets).

Thus the analysis uses five categories: 0-tag, 1-tagL, 1-tagT, 2-tagL, and 2-tagT, where $\mathrm{L}$ and $\mathrm{T}$ subscripts represent loose and tight selection, respectively. The selection requirements are summarized in Table 4.1.

By introducing the sub-samples of 0-tag and loose categories, we considerably increase the number of $t \bar{t}$ candidate events with respect to the previous Matrix Element measurement [11] which used only tight categories with a smaller integrated luminosity $\left(5.6 \mathrm{fb}^{-1}\right)$.

The event selection applied in our analysis is the same used by the last top mass measurement $[9]$ performed with the template method (briefly discussed in section 5.12). However, we apply different additional cuts in the selection, as explained in section 4.5 .

\subsection{Background}

There are several non- $t \bar{t}$ processes which can mimic the signature of the signal of interest, and thus need to be considered as potential background.

$\boldsymbol{W}+$ jets The main contribution to the physical background in the lepton + jets decay channel stems from events in which a $W$ boson is produced together with a number of hadronic jets. Such processes have large cross section and the leptonic $W$ decay can mimic a single lepton $t \bar{t}$ event if the jets are at least four. This background can be divided into $W+H F$ (heavy flavor), i.e. $W+b \bar{b}, W+c$ and $W+c \bar{c}$, and $W+L F$ (light flavor). As $W+H F$ contains a real $W$ and a correct $b$-tag, it is an unavoidable background source. However, the production of real heavy flavor is relatively low, so this background source does not overwhelm our signal. In $W+L F$ a $W$ 
Table 4.1: Selection requirements depending on the number of identified $b$ jets and on the number of tight and loose jets.

\begin{tabular}{lccccc}
\hline & 0 -tag & 1-tagT & 1-tagL & 2-tagT & 2-tagL \\
\hline Lepton $E_{T}[\mathrm{GeV}]$ & $>20$ & $>20$ & $>20$ & $>20$ & $>20$ \\
Lepton $|\eta|$ & $0-1$ & $0-1$ & $0-1$ & $0-1$ & $0-1$ \\
\hline$E_{T}[\mathrm{GeV}]$ & 20 & 20 & 20 & 20 & 20 \\
\hline Leading 3 jets $E_{T}[\mathrm{GeV}]$ & 20 & 20 & 20 & 20 & 20 \\
Leading 3 jets $\eta$ & $0-2$ & $0-2$ & $0-2$ & $0-2$ & $0-2$ \\
\hline $4^{\text {th }}$ jet $E_{T}[\mathrm{GeV}]$ & $>20$ & $>20$ & $>12$ & $>20$ & $>12$ \\
$4^{\text {th }}$ jet $\eta$ & $0-2$ & $0-2$ & $0-2.4$ & $0-2$ & $0-2.4$ \\
\hline Extra jets $E_{T}[\mathrm{GeV}]$ & $<20$ & Any loose & $\begin{array}{c}\text { Any loose } \\
\text { or } \geq 1 \text { tight }\end{array}$ & Any loose & $\begin{array}{c}\text { Any loose } \\
\text { or } \geq 1 \text { tight }\end{array}$ \\
\hline
\end{tabular}

boson is produced in association with jets not containing heavy flavor, but one of the jets is mistagged as a $b$ jet. The amount of this background may be dependent on the mistag performance of our tagging algorithm.

$\mathbf{Q C D}$ There is also a background of non- $W$ multi-jet events. Occasionally jets can release a leptonic signature in the detector because:

- jets may be misidentified as electrons if the energy deposits in the electromagnetic calorimeter are unusually high: these jets are called "fake" electrons;

- secondary electrons can arise from hadron weak decays inside jets and may happen to survive the isolation cut.

Since QCD events are produced in much greater quantities than $W$-containing events, this background is potentially a very large source. However it is reduced significantly by the selection cuts.

There are other sources of background, which represent a smaller contribution but should still be taken into account.

Single top These events contain a real $W$ boson and at least one real $b$ quarks, but the cross section is lower for single top production with respect to $t \bar{t}$ production. These events also need at least one other high-energy jet to mimic the $t \bar{t}$ signature, so these are a relatively smaller contribution. 
Diboson ( $W W, W Z, Z Z)$ In a $W W$ event, one $W$ can decay leptonically and the other hadronically. In $W Z$ events the $Z$ can decay in two charged lepton, with one lepton unobserved, and the $W$ can decay hadronically or the $W$ decays leptonically and the $Z$ decays hadronically. In $Z Z$ events, a $Z$ boson can decay hadronically, while the other decays leptonically. Like single top, these events require extra jets, coming from initial or final state radiation, to mimic the $t \bar{t}$ signature and therefore they have a relatively small cross section.

$Z+$ jets Similarly to $W+$ jets, processes with a leptonically decaying $Z$ boson and associated hadronic jets may fake a semileptonic signal.

\subsection{Monte Carlo Samples}

For signal as well as for background events, we use a variety of Monte Carlo (MC) simulated sample in constructing and evaluating our method. In order to model transfer functions and matrix element, and to calculate the acceptance (see chapter 5 for details), we use $t \bar{t}$ signal samples generated by PYTHia [70] at leading-order (LO), in the range of top masses between $155 \mathrm{GeV}$ and $195 \mathrm{GeV}$. For the calibration procedure (see chapter 7 for explanation) we use $t \bar{t}$ samples accurate at next-to-leading-order (NLO), simulated by using POWHEG [64] with the parton showering performed by PYTHIA. Since the calibration step needs high accuracy, we use a NLO generator and we also enlarge the range of masses used in this procedure with respect to the previous analysis [11 by producing additional MC $t \bar{t}$ samples with top mass in the range $157.5 \mathrm{GeV}$ to $187.5 \mathrm{GeV}$.

MC samples for background are produced by several generators, as listed below.

- We use ALPGEN [48] and PYTHIA, respectively to simulate and shower both $W+$ jets and $Z+$ jets events. These samples are generated with a specific number of partons in the matrix element; for instance, the $W+b \bar{b}$ contribution includes samples with $W+b \bar{b}+0 p, W+b \bar{b}+1 p$, and $W+b \bar{b}+\geq 2 p$, where $p$ indicates partons. In order to get the total $W+b \bar{b}$ contribution, we must add up these three subsamples. With respect to the previous analysis, we produce additional MC samples for $W+$ jets (inclusive in leptons) with low parton $p_{T}$ cutoff, $p_{T}>5 \mathrm{GeV}$, in order to produce the $W+$ jets transfer functions, that were never used before, and thus cover the low $p_{T}$ part of the phase space on which 
the matrix element of $W+$ jets background has to be integrated over (see chapter 5).

- The single top samples are simulated with a top mass of $172.5 \mathrm{GeV}$ using the MADGRAPH/MADEVENT [46] package along with PYTHIA for the parton shower and hadronization.

- For modeling diboson events we use PYTHIA.

As $\mathrm{MC}$ is not able to adequately model the non- $W$ QCD background, we use out-of-signal regions ("sidebands") of our $t \bar{t}$ data sample. Specifically, we select events from the electron and muon data using the same selection requirements as for the $t \bar{t}$ sample, except that we require the lepton to fail the isolation requirement discussed in section 4.2 , i.e. we require isolation $\geq 0.2$ instead of isolation $\leq 0.1$.

Since we have specific needs for our analysis we create our own minintuple ROOT files, starting from the topntuples ${ }^{2}$ of the signal and background located in SAM, the CDF database which stores all MC samples produced for CDF analyses. Minintuples are simplified data formats consistent with our ME analysis code.

\subsection{Selected Sample Composition}

As mentioned above, a certain fraction of the events passing our selection cuts will not be true $t \bar{t}$ events, but rather background. Since the background events do not contain any useful information on the top mass (the single top events, of course, do contain some information but are still not useful if reconstructed as $t \bar{t}$ events), it is necessary to adopt a strategy to minimize their effect on the reconstructed top mass, so we need to know the expected contributions of each of the above background types to the overall observed total. For the determination of the selected sample composition we refer to a widely used method known in internal CDF terminology as Method II For You (Method II in the following) [44]. This method was originally developed for the $t \bar{t}$ cross section measurement [6], where precise background knowledge is very important. The procedure is used to calculate the normalization of processes in the SECVTx tagged lepton+jets data sample. The basic principle of Method II is to use MC simulation to estimate the background contributions from sources where the MC is well understood and models the data well; for channels where the MC is known not to model the data

\footnotetext{
${ }^{2}$ The topntuple is the standard ROOT ntuple format used by CDF for top quark analysis.
} 
contributions well, it employs a data-based approach (see reference [44] for details).

Since we exploit the same dataset used by the previous CDF template analysis $[9]$ and we apply the same basic selection, we don't need to run Method II, but we can refer to the results previously obtained and collected in pre-existing tables $57 \mid$ which fit our sample. To be precise, in our analysis we use a slightly different Good Run List (GRL, introduced in section 2.2.7). As a result the total integrated luminosity corresponds to $9.0 \mathrm{fb}^{-1}$, while the previous template measurement exploited a slightly smaller dataset of $8.7 \mathrm{fb}^{-1}$. Therefore the numbers in Method II tables should be scaled according to this. The Method II results consist of several estimates. For each background subsample the number of expected events is derived for each jet multiplicity (1 to 5 tight jets) and each tagged category (0-tag, 1-tag and 2-tag). Also, since the trigger and lepton identification (lepton ID) efficiencies depend on different subdetectors, the estimates are given for each lepton category (CEM electrons, CMUP muons, CMX muons, loose muons).

The number of expected events has to be corrected by an efficiency term of additional cuts applied beyond the basic cuts shown in Table 4.1. As additional selection in our analysis we just apply QCD veto, based on the algorithm that rejects a non- $W$ event if it has $\mathbb{E}_{T}<30 \mathrm{GeV}$ and if the azimuthal angle between $\vec{E}_{T}$ and the leading jet direction is $<0.5$ or $>2.5$ radiants. However, we found that too many signal events are rejected for 2 -tag events even though background rejection is small. This is because we already achieve very good signal-to-background ratio in the 2-tag sample. Therefore we apply the QCD multijet background rejection only for 0-tag and 1-tag events.

The cut efficiency is calculated as the ratio between the sum of the weight of the events after the cut and the sum of weights before cut, where the event weight is the product between the trigger efficiency, the lepton ID scale factor and the integrated luminosity. The number of expected events for each background is evaluated by multiplying the Method II estimate and the cut efficiency, and adding up numbers obtained for each subdetector. For the signal events estimation, PYTHIA sample with $m_{t}=172.5 \mathrm{GeV}$ is used. In order to evaluate the number of observed events we just account those events which pass the basic selection requirements and the QCD veto for each category. The sample composition is shown in Table 4.2 . 
Table 4.2: Expected and observed sample composition.

\begin{tabular}{cccccc}
\hline & 0-tag & 1-tagL & 1-tagT & 2-tagL & 2-tagT \\
\hline Wbbbar & 125.6 & 177.1 & 82.2 & 27.3 & 17.0 \\
Wccbar & 384.8 & 112.9 & 52.5 & 4.1 & 2.6 \\
Wc & 186.7 & 66.8 & 25.9 & 2.3 & 1.3 \\
W+light j & 1580.9 & 170.7 & 77.2 & 2.9 & 1.9 \\
Z+jets & 169.4 & 25.2 & 13.7 & 2.0 & 1.3 \\
single top & 13.9 & 16.5 & 8.2 & 6.7 & 4.6 \\
Diboson & 166.3 & 31.0 & 17.9 & 2.7 & 1.8 \\
QCD & 623.2 & 119.9 & 60.3 & 60.3 & 6.3 \\
\hline Total Bkg & 3250.8 & 720.1 & 337.9 & 49.0 & 36.8 \\
\hline ttbar & 959.7 & 998.6 & 1086.3 & 331.3 & 425.5 \\
\hline \hline Expected total & 4210.5 & 1718.7 & 1424.2 & 380.3 & 462.3 \\
Observed & 4474 & 1711 & 1434 & 365 & 375 \\
\hline
\end{tabular}

In general, we observe a good agreement between the number of total expected events and the number of observed events. Only in the 2-tagT category we note a substantial difference of $\sim 20 \%$ : in this case the number of expected events exceeds the number of observed events. On the contrary in 0-tag sample the number of expected events is mildly smaller than the number of observed events. The total signal-to-background ratio, obtained by summing up the overall signal and background and calculating the ratio, is $\mathrm{Sig} / \mathrm{Bkg} \sim 1 / 1$.

In order to verify the correctness of the method used to evaluate the sample composition, we apply to our samples the same additional cuts of the previous template analysis and we make a cross check with their results. Our results are in good agreement with the previous analysis, excepting for the QCD sample estimation which is differently modeled in the two analysis. The previous template analysis models QCD by using anti-electron sample [43], whereas we use the inverted isolation cut described above.

The signal-to-background ratio obtained by applying the same cuts of the previous analysis is $\mathrm{Sig} / \mathrm{Bkg} \sim 2 / 1$ showing that in our analysis $(\mathrm{Sig} / \mathrm{Bkg} \sim$ $1 / 1$ ), where we apply only the QCD veto as additional cut, the background is larger, mostly for 0-tag and 1-tag categories. However, to improve Sig/Bkg we shall apply an additional cut on the final likelihood. 


\subsection{Validation of Data}

Since this analysis is necessarily reliant on MC simulation, we made a large number of validation plots, to show that the observed features of our data agree well with the MC. This work was essential to prove the soundness of the analysis and was a significant part of my own effort. The plots illustrated in the following pages show some quantities of interest plotted for the data against $\mathrm{MC}$ events of the samples described in section 4.4. The $t \bar{t}$ signal sample used here is the PYTHIA sample with $m_{t}=172.5 \mathrm{GeV}$. The MC distributions of each sample are normalized with the number of expected events obtained in section 4.5 and summarized in Table 4.2.

Every set of plot contains the specific kinematic distribution of each category, in order: 0-tag, 1-tagT, 1-tag-L, 2-tagT, 2-tagL.

- Figure 4.2 shows the distribution of the lepton transverse momentum $p_{T}$. The comparison between data and MC for 0-tag sample shows some disagreement in the shape of the distributions. In the first row of figure 4.2 the distribution of 0 -tag sample (top left) is compared to the distribution obtained by removing the QCD background and rescaling data with the number of background events (top right). The latter shows a better agreement between data and $\mathrm{MC}$, indicating that the modeling of the QCD background should be refined. This item is currently under discussion.

- Figure 4.3 shows the distributions of the lepton pseudorapidity.

- Figure 4.4 shows the distributions of the quantity $H_{T}$ which represents the scalar sum of transverse energies in the event, $H_{T}=E_{T}^{l}+\mathbb{E}_{T}+$ $\sum_{\text {jets }} E_{T}^{j e t}$, where $E_{T}^{l}$ is the transverse energy of the lepton, $\mathbb{E}_{T}$ the scalar missing energy, and $E_{T}^{\text {jet }}$ the transverse energy of the jet. Even though $H_{T}$ cut is applied in many analysis, we don't use $H_{T}$ since it has a top mass dependent distribution.

- In Figure 4.5 the distribution of the missing transverse energy $\mathbb{E}_{T}$ is shown. For 0 -tag and 1-tag the distribution has a peak corresponding to $\mathbb{E}_{T}=30 \mathrm{GeV}$. This is due to the QCD veto applied for events with $\mathbb{E}_{T}<30 \mathrm{GeV}$.

- The distributions in Figure 4.6 represent the transverse momentum of the $b$-jets. If we can't reconstruct the $b$-jets from the secondary-vertex tagging algorithm we exploit the results of the kinematic reconstruction 
performed by the previous template analysis [9]. The template analysis is based on the reconstruction of some specific top quark mass estimator. Every selected event is subjected to a kinematic fit, and the jet-parton assignment that yields the best $\chi^{2}$ for a $t \bar{t}$ hypothesis is chosen to compute the estimator of the top quark mass. In particular, the jets showing the best $\chi^{2}$ under the $b$-flavor hypothesis are considered as $b$-jets [55].

- In Figures 4.7 and 4.8 the distributions in transverse energy and in pseudorapidity of the first four jets are reported.

- The last set of plots in Figure 4.9 shows the distribution of the transverse mass of the $W$ boson defined as $M_{T}^{W}=\frac{1}{c^{2}} \sqrt{2 \cdot E_{T}^{l} \cdot E_{T} \cdot\left(1-\cos \phi_{l \nu}\right)}$, where $\phi_{l \nu}$ is the angle between the lepton and the $\mathbb{E}_{T}$ vector in the transverse plane.

In general, a good agreement between data and $\mathrm{MC}$ simulations is observed in all categories, excepting the 2 -tagT category in which we notice that data undershoot $\mathrm{MC}$ simulations. The reason is that the $\mathrm{MC}$ distributions are normalized by the number of expected events which are not in good agreement with the number of observed events in data, as shown in Table 4.2 . For the same reason in 0-tag category, data overshoot mildly the $\mathrm{MC}$ distributions. However, the ME method is insensitive to the absolute normalization of the various categories and sensitive to the shapes of the distributions which seem to be in good agreement in almost every case, excepting in the lepton $p_{T}$ distribution. 


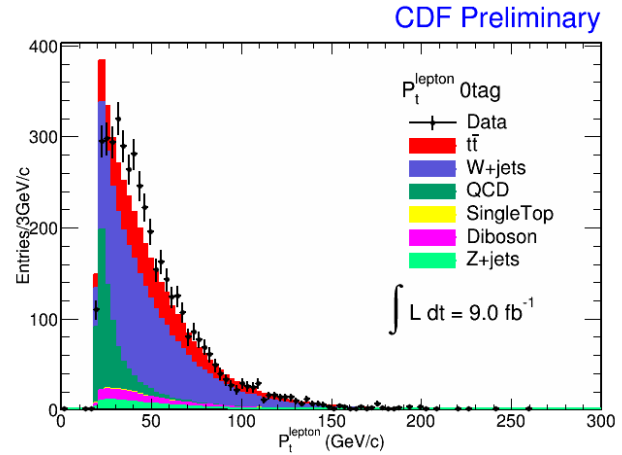

(a)

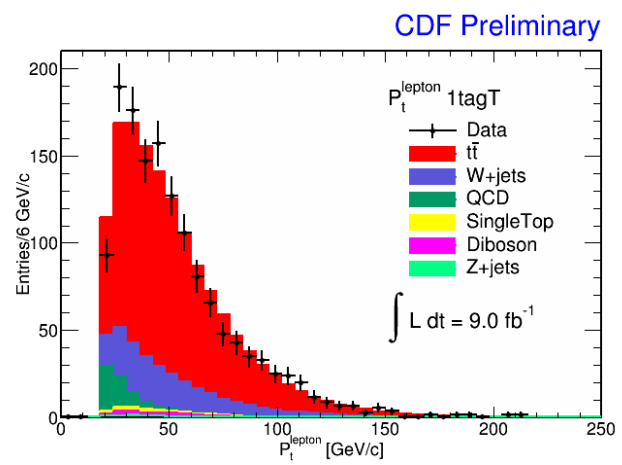

(c)

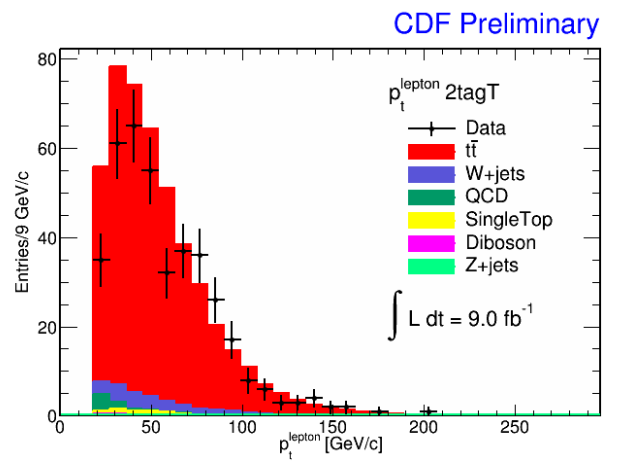

(e)

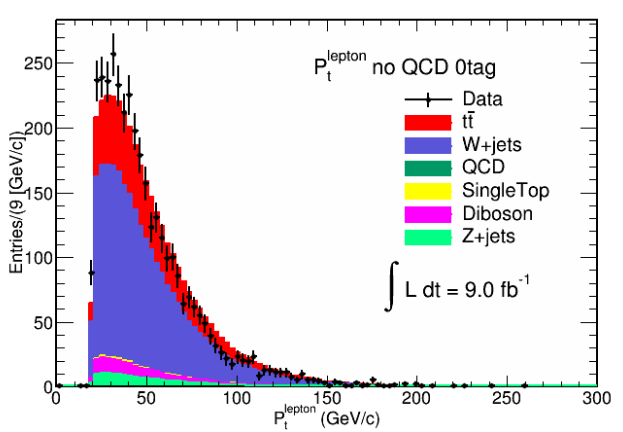

(b)

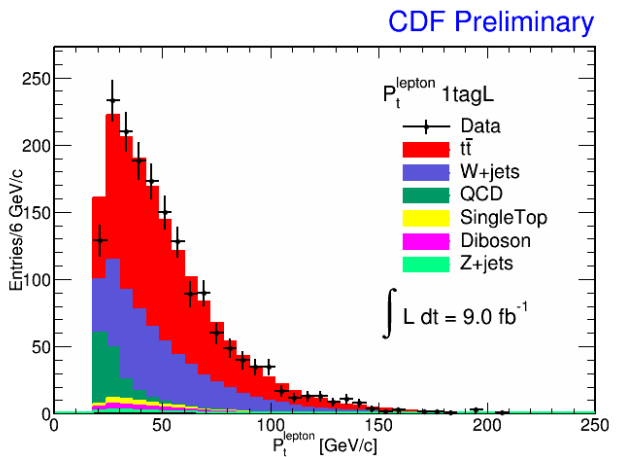

(d)

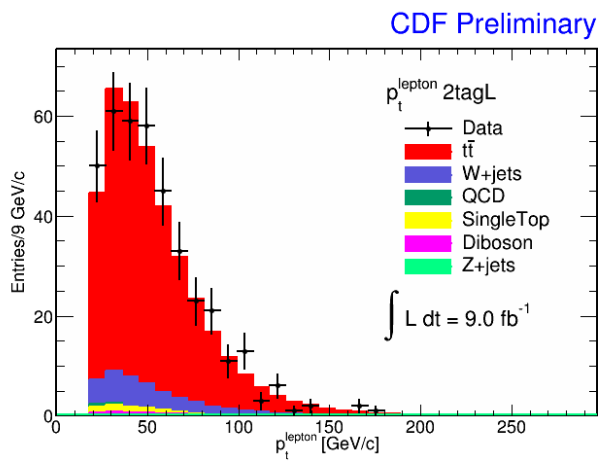

(f)

Figure 4.2: Comparison of lepton $p_{T}$ between data and MC for events passing our selection cuts. 


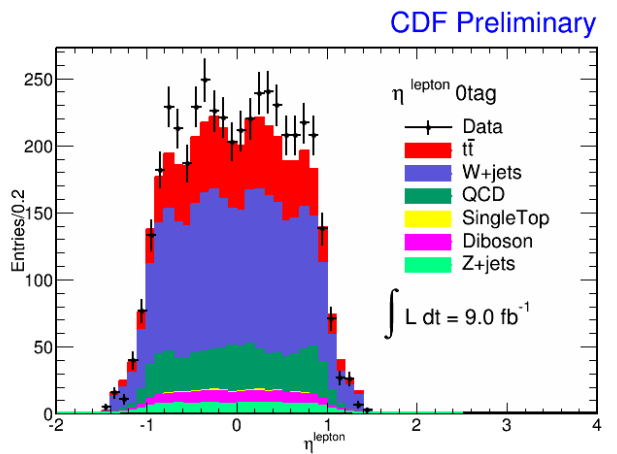

(a)

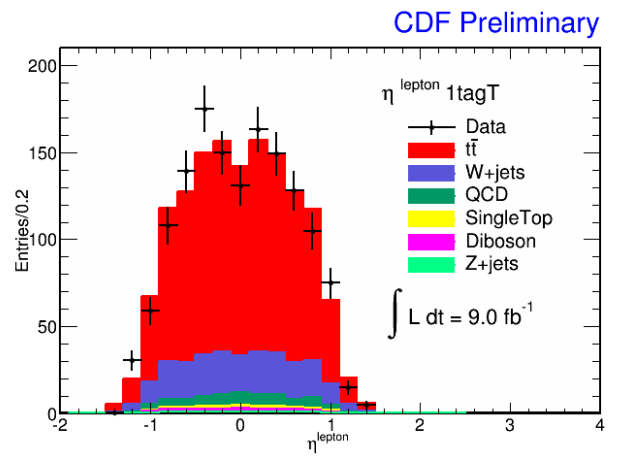

(b)

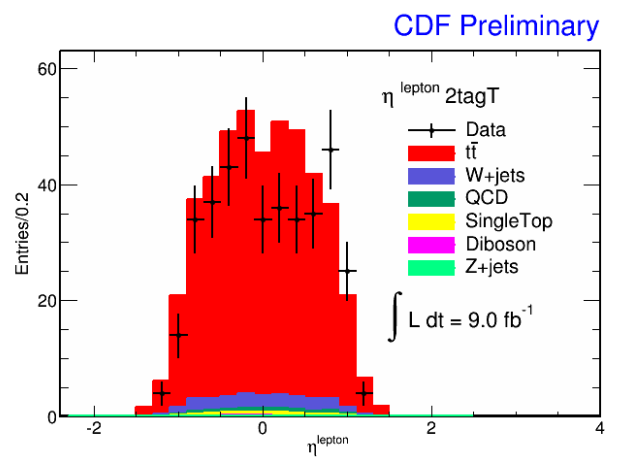

(d)

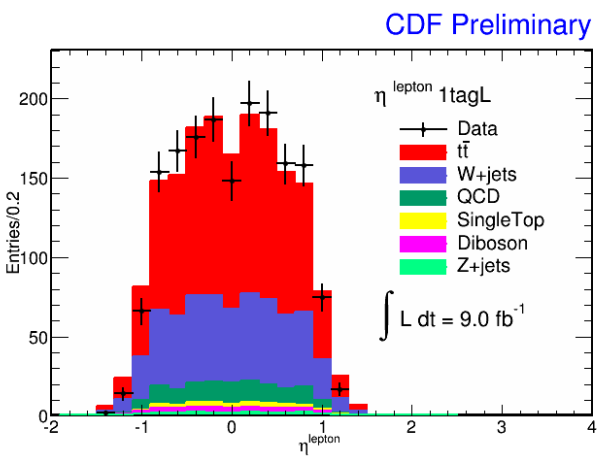

(c)

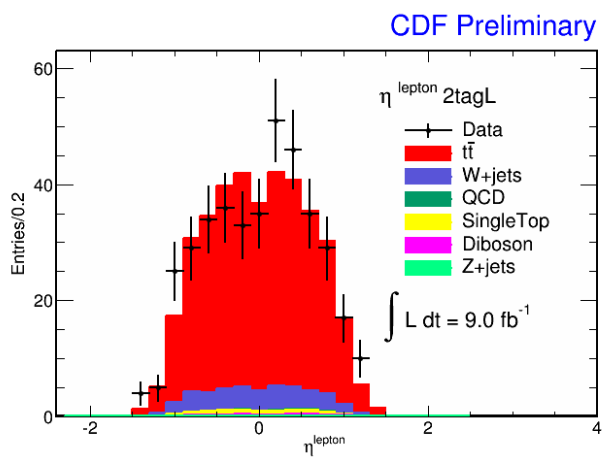

(e)

Figure 4.3: Comparison of lepton $\eta$ between data and MC for events passing our selection cuts. 


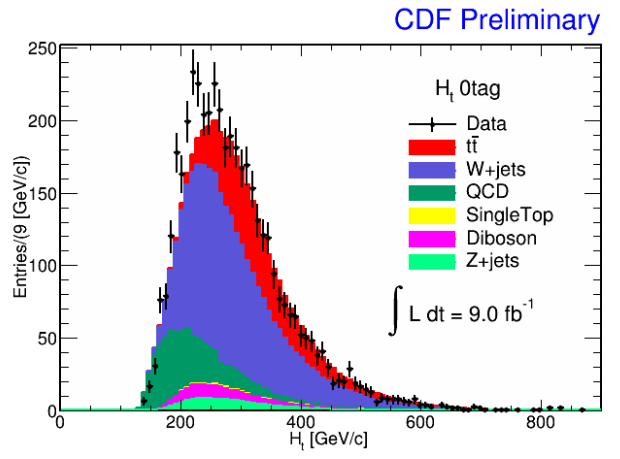

(a)

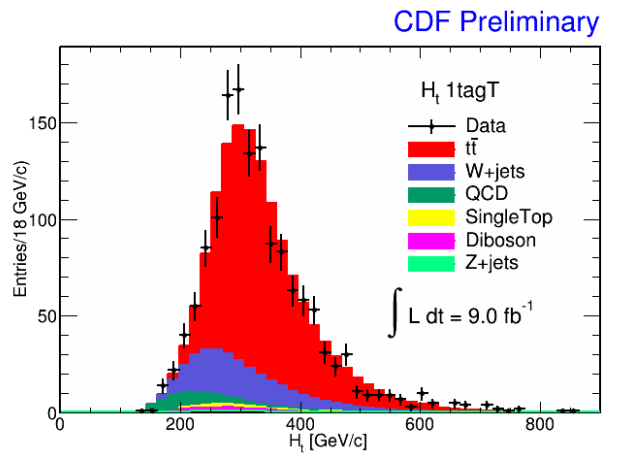

(b)

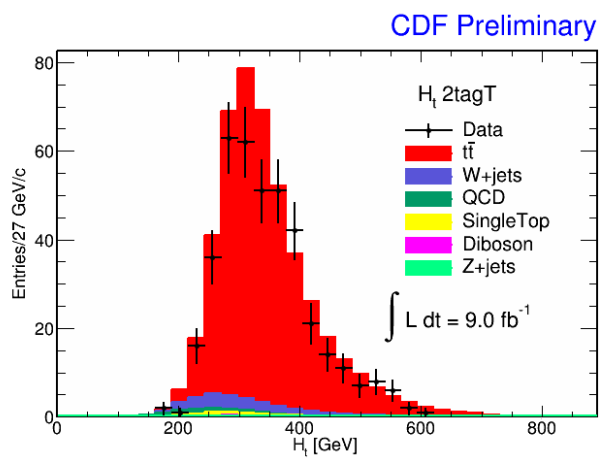

(d)

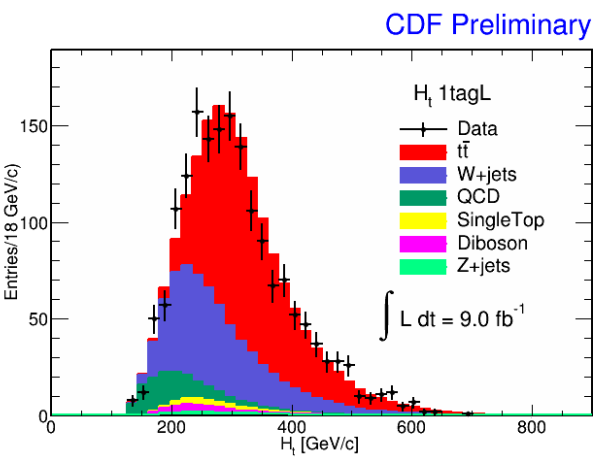

(c)

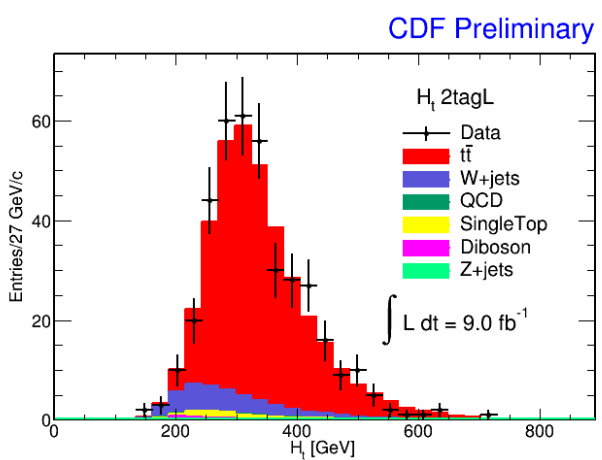

(e)

Figure 4.4: Comparison of $H_{T}$ between data and MC for events passing our selection cuts. 


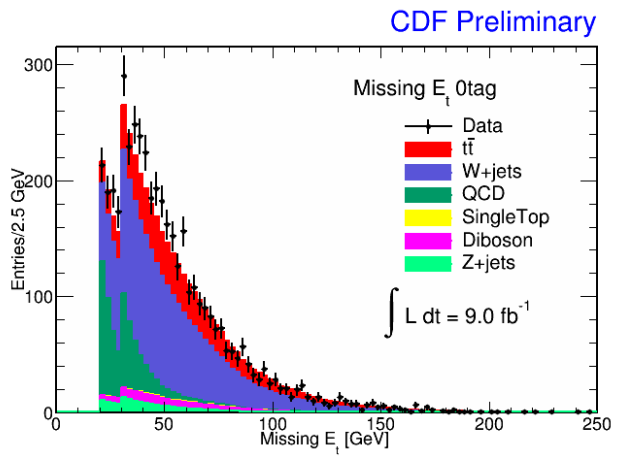

(a)

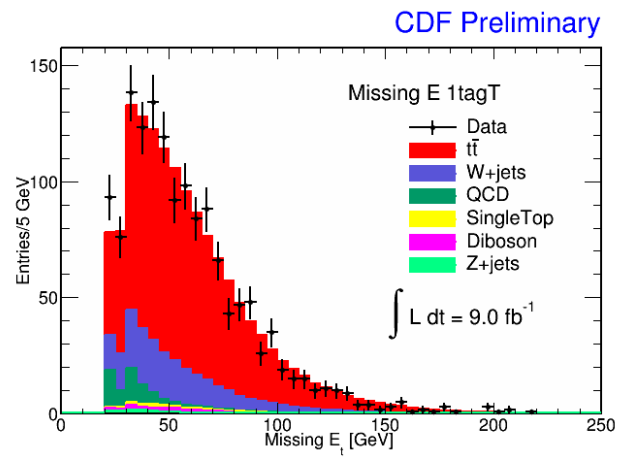

(b)

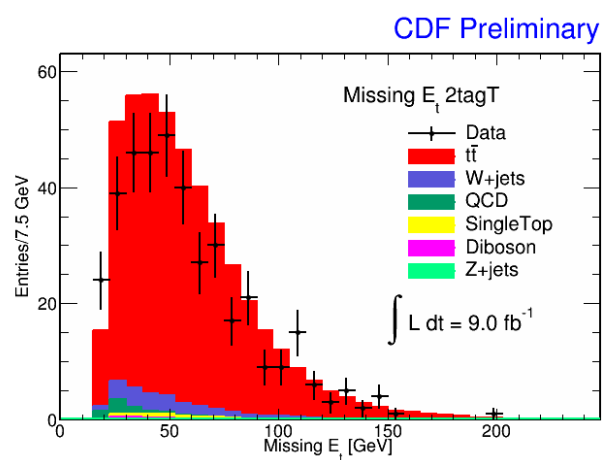

(d)

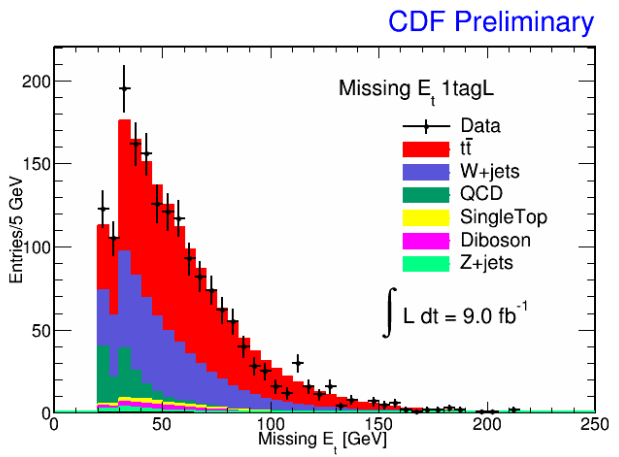

(c)

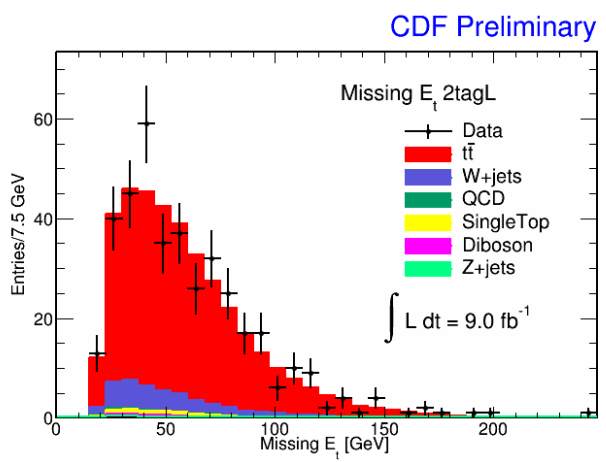

(e)

Figure 4.5: Comparison of $\mathbb{E}_{T}$ between data and MC for events passing our selection cuts. 


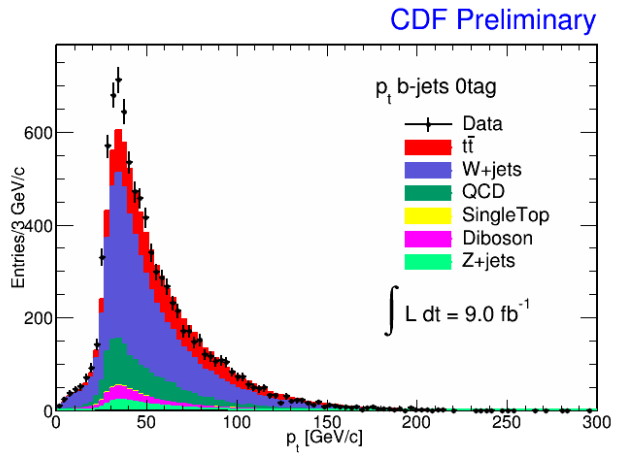

(a)

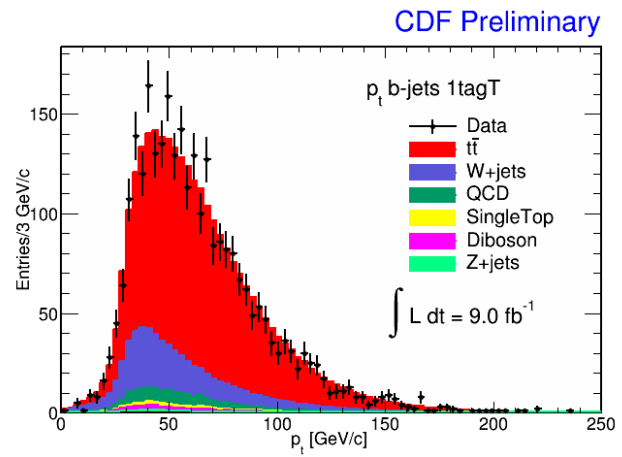

(b)

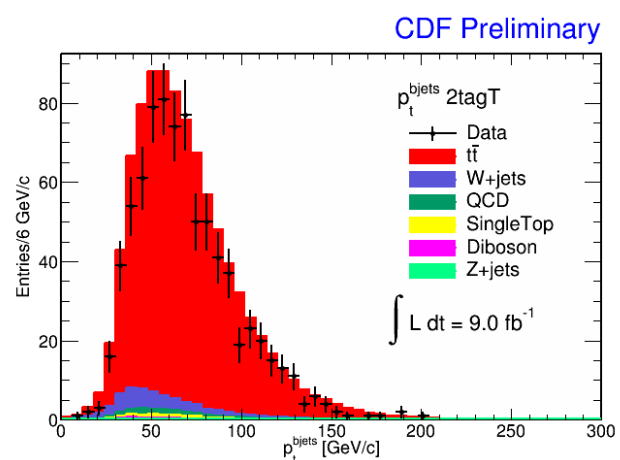

(d)

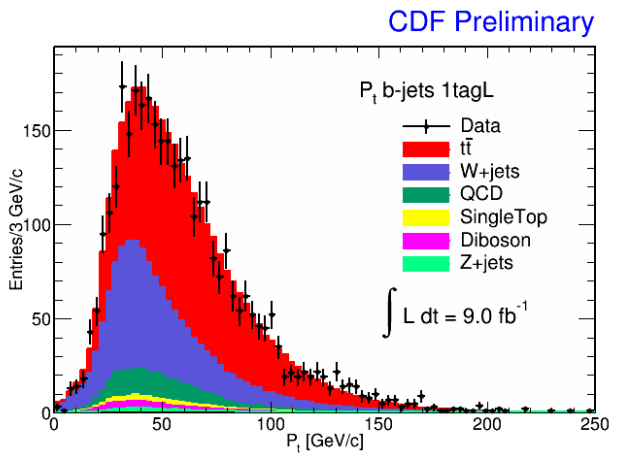

(c)

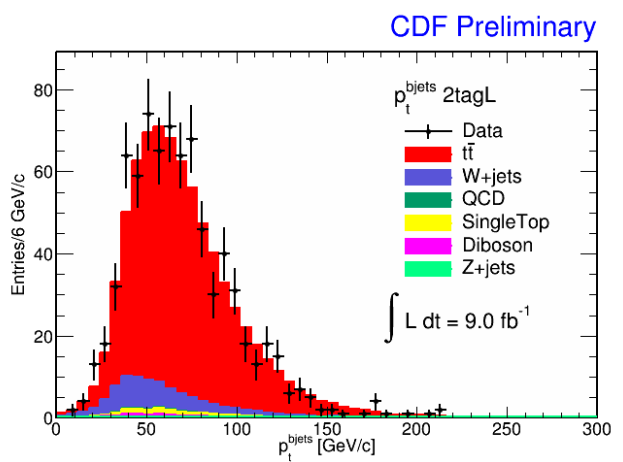

(e)

Figure 4.6: Comparison of $p_{T}$ for the $b$-jets between data and $\mathrm{MC}$ for events passing our selection cuts. 


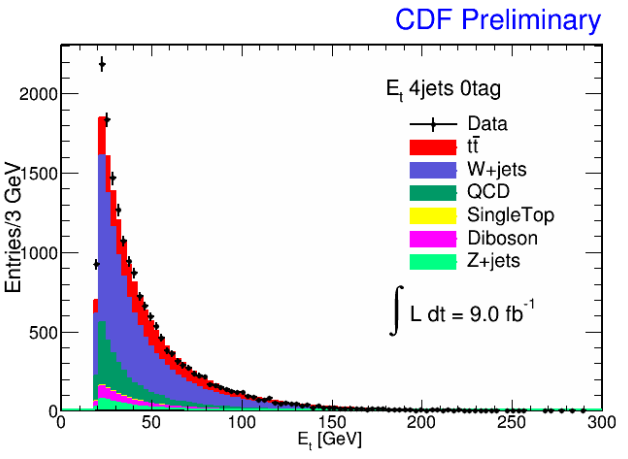

(a)

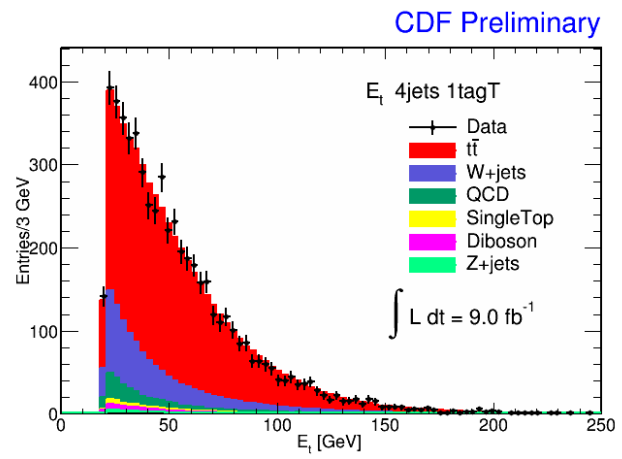

(b)

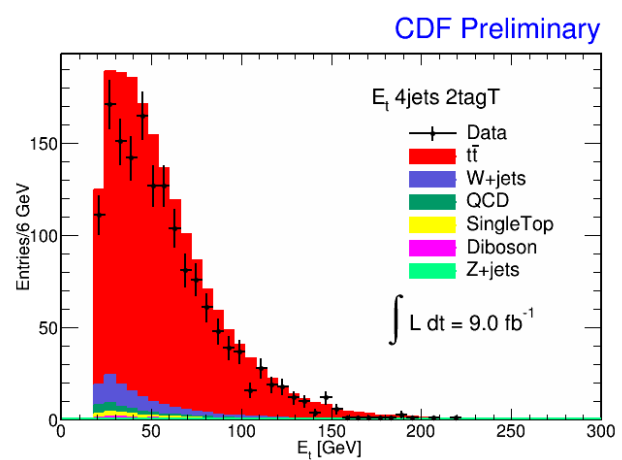

(d)

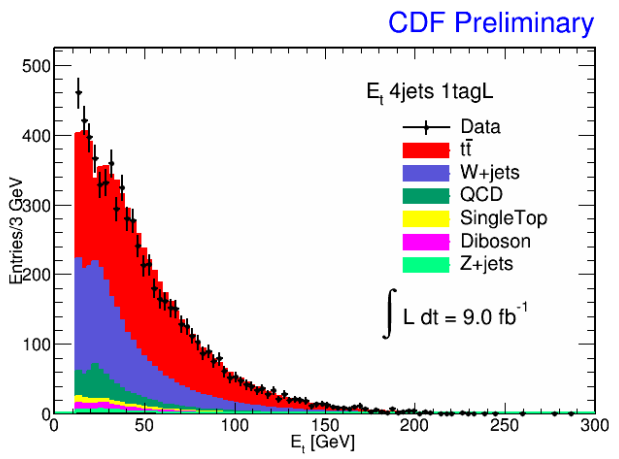

(c)

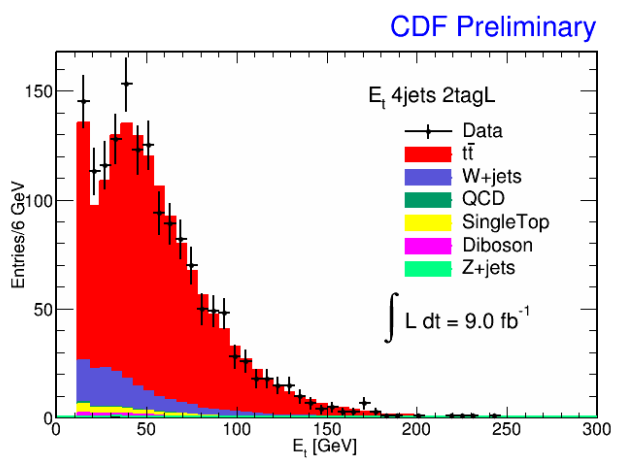

(e)

Figure 4.7: Comparison of $E_{T}$ for the first four jets between data and MC for events passing our selection cuts. 


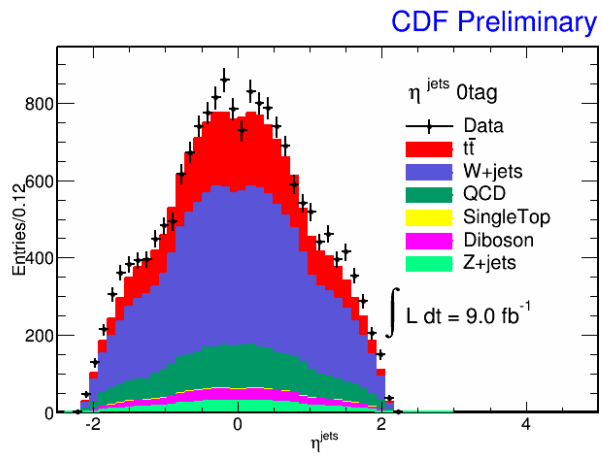

(a)

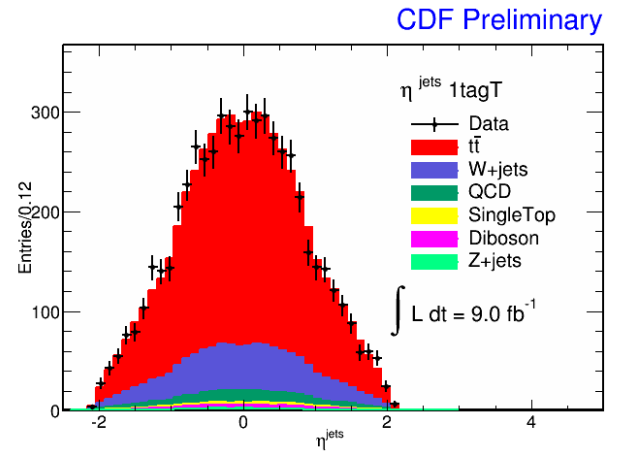

(b)

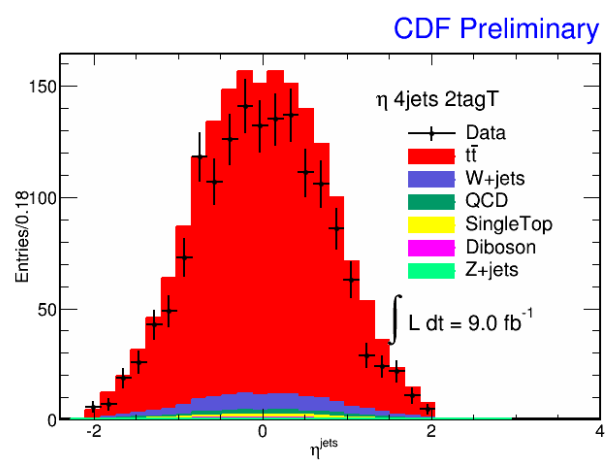

(d)

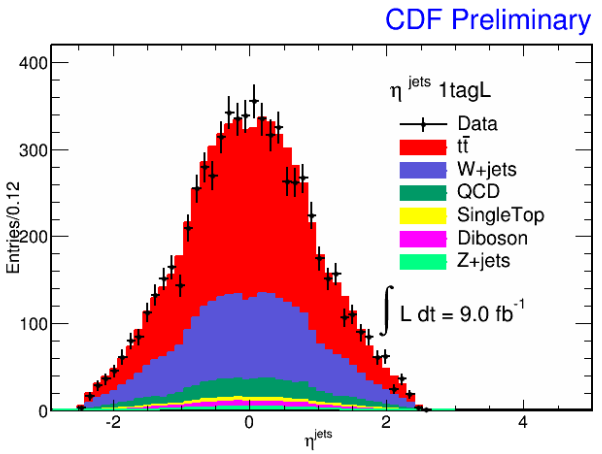

(c)

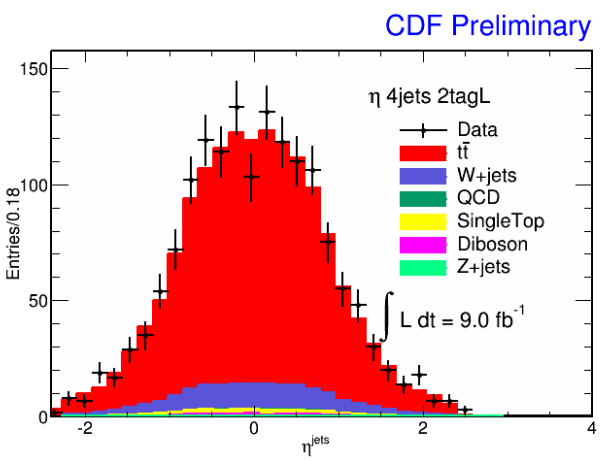

(e)

Figure 4.8: Comparison of $\eta$ for the first four jets between data and MC for events passing our selection cuts. 


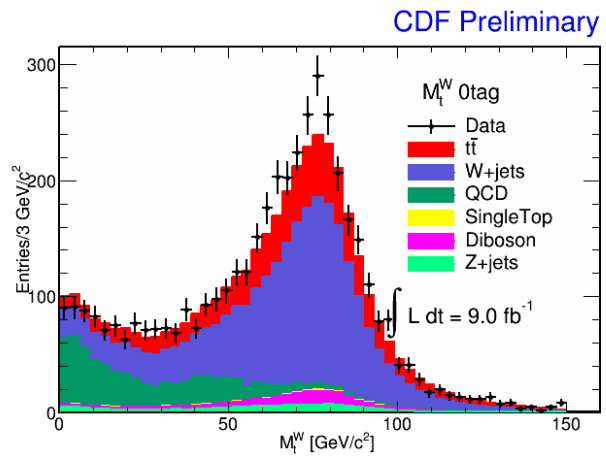

(a)

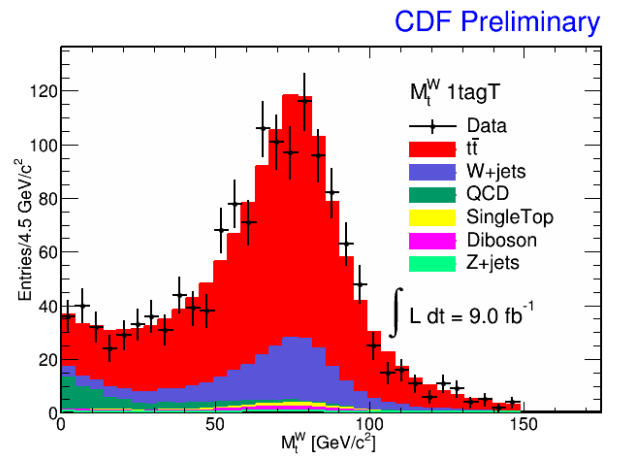

(b)

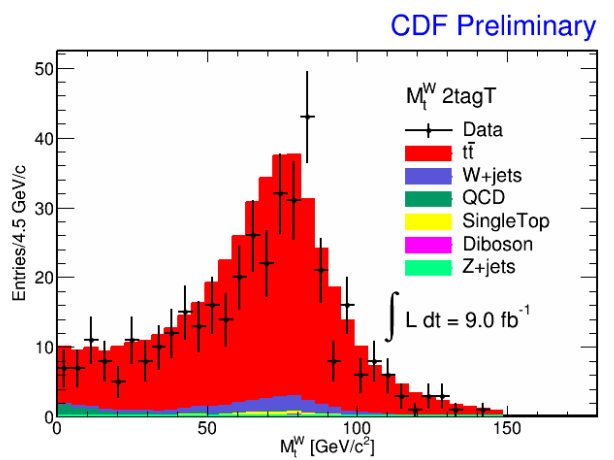

(d)

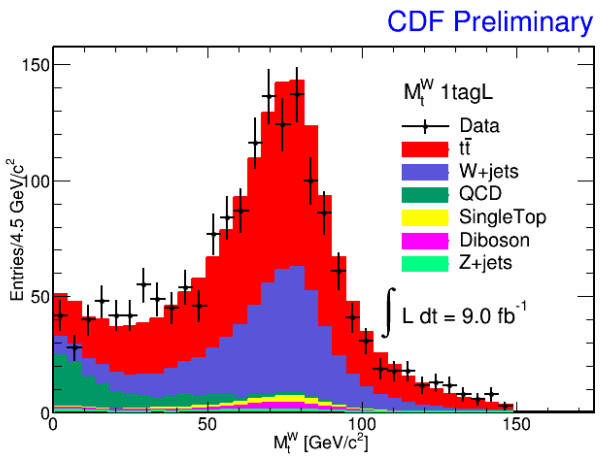

(c)

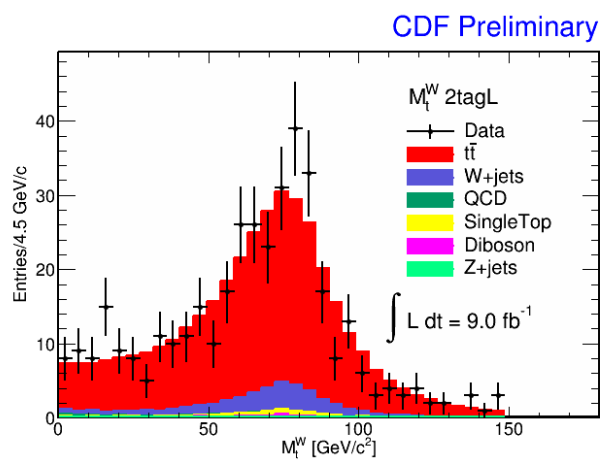

(e)

Figure 4.9: Comparison of $M_{T}^{W}$ between data and MC for events passing our selection cuts. 


\section{Chapter 5}

\section{Matrix Element Method}

\subsection{Introduction to the Matrix Element Method}

The Matrix Element (ME) method is a powerful tool in experimental particle physics, especially at hadron colliders, as it provides a superior statistical sensitivity in the extraction of important parameters of the Standard Model. The key concept of the ME method is to build an event probability assuming two processes, one signal and one background, starting from the entire kinematic information associated to every single selected event. The increased statistical sensitivity of this method with respect to other methods based on distribution fitting, like the widely used template methods, is due to the completeness of the information exploited in each event. This represents the fundamental difference with respect to methods which are sensitive only to one or more distributions of a whole set of events, where each event enters with the same weight. Moreover, the computation of the matrix element checks the full kinematics of the event, as opposed to exploiting information from only a few variables.

The ME method was first suggested by K.Kondo [60] with the purpose to exploit at best the full picture of the $4 \pi$ detectors to give overall picture of individual events. The method was then applied in practice in the pioneering Run I measurement of the top quark mass by the D $\emptyset$ collaboration [16 at the Tevatron. Since then the ME method has been used in studies of top quark properties as well as of b-physics at the Tevatron. It was applied in the measurement of the $\mathrm{W}$ helicity [17] and in the search for single top quark production [7][18]. A notable recent application has been in the study of the Higgs process $H \rightarrow Z Z^{*} \rightarrow 4 l$, where the ME method, in the form of the "MELA" approach, was used in the Higgs discovery by CMS experiment [14. 
The method can in principle be used for any measurement, with the largest gain compared to cut based analysis techniques expected for processes involving intermediate resonances and leading to many particle final states. In general, the ME method, as well as other techniques, can be used to determine several unknown parameters (theoretical parameters describing the physics processes measured as well as experimental parameters describing the detector response) at the same time in one measurement, thus also allowing for a reduction of systematic uncertainties. Our measurement in $t \bar{t}$ events containing one leptonic and one hadronic $W$ decay exploits the known $W$ mass to constrain the energy scale for jets and significantly reduce the main systematic uncertainty of early measurements of the top quark mass. Together with the top quark mass, a simultaneous additional measurement of the jet energy scale can be thus incorporated naturally in the ME method.

The superior sensitivity of the ME technique is achieved by taking into account the full topological and kinematic information in a given event, and determining the probabilities $P_{\text {sig }}(\boldsymbol{y} \mid \boldsymbol{a})$ that an observed event is a signal event, where $\boldsymbol{y}$ is a vector describing the parameters of the $t \bar{t}$ generation model, inferred from detector response, and $\boldsymbol{a}$ is the vector of model parameters. Even though we call $\boldsymbol{y}$ "observed" quantity, it is usually a product of sophisticated event reconstruction procedure which involves pattern recognition, tracking, clustering of jets, kinematic fitting and so on. In the context of the top mass measurement, the parameter set $\boldsymbol{a}$ includes $m_{t}$ and $\Delta_{J E S}$ shift relative to the nominal jet energy scale.

The method can be generalized to encompass background by considering the probability density for an event to coming from background, $P_{b k g}(\boldsymbol{y} \mid \boldsymbol{b})$, where $\boldsymbol{b}$ parameter is referred just to $\Delta_{J E S}$.

In the specific context of our analysis, assuming two non-interfering processes, $t \bar{t}$ signal and $W+$ jets background, the probability distribution per event is defined as

$$
P_{e v}\left(\boldsymbol{y} \mid m_{t}, \Delta_{J E S}\right)=A(\boldsymbol{y})\left[f P_{s i g}\left(\boldsymbol{y} \mid m_{t}, \Delta_{J E S}\right)+(1-f) P_{W j e t s}\left(\boldsymbol{y} \mid \Delta_{J E S}\right)\right]
$$

where $A(\boldsymbol{y})$ term accounts for acceptance and efficiencies and $f$ is the signal fraction which has to be determined by calibration with simulated measurements ("pseudo-experiments"). We would consider $f$ as a function of $m_{t}$ and $\Delta_{J E S}$ if we want to take into account different expected sample composition for different $m_{t}$ and $\Delta_{J E S}$ values. In Equation $5.1 P_{s i g}$ describes the probability density for $t \bar{t}$ production and $P_{W j e t s}$ describes the probability 
density for $W+$ jets production which represents the dominant background contribution. We don't explicitly take into account other backgrounds in $P_{e v}\left(\boldsymbol{y} \mid m_{t}, \Delta_{J E S}\right)$. These small backgrounds will be included in the pseudoexperiments and therefore will be accounted for by some smearing of the $W+$ jets probability function.

For a given sample of $N$ selected events, the parameters to be measured are determined as those values that maximize the likelihood which is constructed at each grid point in $\left(m_{t}, \Delta_{J E S}\right)$ in the measurement space $\boldsymbol{Y}$ from the product of the individual $P_{e v}$ values according to:

$$
L\left(\boldsymbol{Y} \mid m_{t}, \Delta_{J E S}\right)=\prod_{i=1}^{N} P_{e v}\left(\boldsymbol{y}_{i} \mid m_{t}, \Delta_{J E S}\right)
$$

Usually, instead of maximizing the likelihood itself, one minimizes the negative logarithm of the likelihood

$$
-\ln L\left(\boldsymbol{Y} \mid m_{t}, \Delta_{J E S}\right)=-\sum_{i=1}^{N} \ln P_{e v}\left(\boldsymbol{y}_{i} \mid m_{t}, \Delta_{J E S}\right) .
$$

Obtaining a consistent $m_{t}$ estimate is guaranteed by an important property of the Breit-Wigner shape of the top quark mass distribution (which limits the $m_{t}$ resolution that can be achieved in a single even by a perfect detector): the slope of its log-likelihood is bounded. This means that a contaminating event inconsistent with the model can only make a finite contribution to the determination of top quark mass. To give an idea that this is not always the case, let us consider the determination of the mean when the density is Gaussian. In this case, the maximum likelihood analysis leads to the conclusion that the optimal value of the mean should be determined by simply averaging all observations. In this circumstance even a single observation not described by the model (many standard deviations away from the "real" mean) can give arbitrarily large contributions to the average. On the contrary, if the ME analysis does not employ the narrow width approximation for the top quark, the maximum likelihood estimate of $m_{t}$ is guaranteed to be robust and can tolerate certain deficiencies in the background modeling [33].

The Matrix Element Method can be understood as an application of the Bayesian principle of integrating over all unobserved degrees of freedom with a well motivated informative prior provided by the Standard Model theory. In fact, if we perfectly knew all the variables related to the particles involved in a $t \bar{t}$ event, with the exception of the quantity of interest, it would be 
straightforward to evaluate the matrix element for $t \bar{t}$ production and decay for a variety of possible $m_{t}$ and thus build a likelihood curve of observing that event in the detector as a function of $m_{t}$. The reality is very different indeed, as we don't perfectly know the variables involved in the processes because they are obtained by measurements that are always subject to errors and sometimes impossible. What we can do is to integrate over all of the unknown variables, making use of appropriate prior reflecting our knowledge of the expected values of the variables, related through the transfer functions to the known quantities measured in the detector. The prior is provided by the full dynamical model of $t \bar{t}$ production and decay, defined in the 32 dimensional phase space of two initial and six final state particles, as predicted by the Standard Model. This adopted dynamical model is the leading order expression of the theory. Corrections for higher order effects are applied after a calibration of the method. In a similar way $W+4 j e t s$ events are treated. Hence our mass measurement is valid only under the assumption that the properties of the top quark (and $W+4 j e t s$ ) are correctly described by the Standard Model.

The main components of the prior are the matrix element and the phase space terms: for this reason the technique is referred to as the "Matrix Element Method". The main components of the matrix element are the propagators for top quark and $\mathrm{W}$ boson which lead to the characteristic Breit-Wigner shape of the mass distributions.

\subsection{Signal Probability}

The probability density for $t \bar{t}$ production to yeld a given set of partonic final state four-momenta $\boldsymbol{x}$ in the hard scattering of two partons with fourmomenta $q_{1}$ and $q_{2}$ is proportional to the differential cross section $d \sigma$ of the corresponding process, given by

$$
d \sigma_{q \bar{q}, g g \rightarrow t \bar{t}}\left(\boldsymbol{x}, m_{t}\right)=\frac{(2 \pi)^{4}\left|M_{q \bar{q}, g g \rightarrow t \bar{t}}\right|^{2}}{2 g_{\mu \nu} q_{1}^{\mu} q_{2}^{\nu}} d \Phi(\boldsymbol{x})
$$

where $M_{q \bar{q}, g g \rightarrow t \bar{t}}$ denotes the matrix element of the $t \bar{t}$ production through quark-antiquark annihilation and gluon-gluon fusion and the subsequent decay in lepton + jets channel $q \bar{q}, g g \rightarrow t \bar{t} \rightarrow b(l \nu) \bar{b}\left(q \overline{q^{\prime}}\right), g_{\mu \nu}$ is the tensor of the Minkowski metric, and $d \Phi(\boldsymbol{x})$ is an infinitesimal element of the phase space for the eight particles in the $t \bar{t}$ production and decay process [20].

To obtain the differential cross section in $p \bar{p}$ collisions, the expression in Eq.5.4 is convoluted with the corresponding probability distributions for all 
possible flavor combinations of the colliding quark and antiquark, as well as the probability distribution of incoming gluons, yielding

$$
d \sigma_{p \bar{p} \rightarrow t \bar{t}}\left(\boldsymbol{x}, m_{t}\right)=\sum_{\substack{g g, q \bar{q} \text { flav }}} \int d \boldsymbol{q}_{\mathbf{1}} d \boldsymbol{q}_{\mathbf{2}} f\left(\boldsymbol{q}_{\mathbf{1}}\right) f\left(\boldsymbol{q}_{\mathbf{2}}\right) d \sigma_{q \bar{q}, g g \rightarrow t \bar{t}}
$$

where $f\left(\boldsymbol{q}_{\mathbf{1}}\right)$ and $f\left(\boldsymbol{q}_{\mathbf{2}}\right)$ are the Parton Distribution Functions (PDFs) describing the momenta $\boldsymbol{q}_{\mathbf{1}}$ and $\boldsymbol{q}_{\mathbf{2}}$ carried by the incoming partons.

In general, the set $\boldsymbol{y}$ of measured four-momenta is not identical to the set of corresponding partonic variables $\boldsymbol{x}$ because of finite detector resolution and the evolution of quarks into jets. This is taken into account through the transfer function $(\mathrm{TF}) W\left(\boldsymbol{y} \mid \boldsymbol{x}, \Delta_{J E S}\right)$, representing the probability density to observe detector response $\boldsymbol{y}$ given the parton level momentum $\boldsymbol{x}$ and a $J E S$ shift $\Delta_{J E S}$. Thus, the probability to observe a given reconstructed $t \bar{t}$ event characterized by $\boldsymbol{y}$ is obtained through a convolution with the TF in the calculation of the differential cross section

$$
d \sigma_{p \bar{p} \rightarrow t \bar{t}}\left(\boldsymbol{y} \mid m_{t}, \Delta_{J E S}\right)=\int_{\boldsymbol{x}} d \boldsymbol{x} d \sigma_{p \bar{p} \rightarrow t \bar{t}}\left(\boldsymbol{x} \mid m_{t}\right) \times W\left(\boldsymbol{y} \mid \boldsymbol{x}, \Delta_{J E S}\right) .
$$

The probability distribution to observe a $t \bar{t}$ event with a set of kinematic quantities $\boldsymbol{y}$ in the detector is given by

$$
P_{s i g}\left(\boldsymbol{y} \mid m_{t}, \Delta_{J E S}\right)=\frac{d \sigma_{p \bar{p} \rightarrow t \bar{t}}\left(\boldsymbol{y} \mid m_{t}, \Delta_{J E S}\right)}{\sigma_{p \bar{p} \rightarrow t \bar{t}, a c c}\left(m_{t}, \Delta_{J E S}\right)}
$$

where the total cross section for $t \bar{t}$ production observed in the detector, defined as

$$
\sigma_{p \bar{p} \rightarrow t \bar{t}, a c c}\left(m_{t}, \Delta_{J E S}\right)=\int_{\boldsymbol{y}} d \boldsymbol{y} A(\boldsymbol{y}) d \sigma_{p \bar{p} \rightarrow t \bar{t}}\left(\boldsymbol{y} \mid m_{t}, \Delta_{J E S}\right)
$$

ensures that $A(\boldsymbol{y}) P_{\text {sig }}$ is normalized to unity, where $A(\boldsymbol{y})$ is a term that accounts for the fraction of events that gets detected and passes the selection cuts.

Using the previous equations, the probability distribution to observe a $t \bar{t}$ event with four-momenta $\boldsymbol{y}$ in the detector can be written explicitly as

$$
\begin{aligned}
P_{s i g}\left(\boldsymbol{y} \mid m_{t}, \Delta_{J E S}\right) & =\frac{1}{\sigma_{p \bar{p} \rightarrow t \bar{t}, \text { obs }}} \times \sum_{\substack{g g, q \bar{q} \text { flav }}} \int\left[d \boldsymbol{q}_{\mathbf{1}} d \boldsymbol{q}_{\mathbf{2}} f\left(\boldsymbol{q}_{\mathbf{1}}\right) f\left(\boldsymbol{q}_{\mathbf{2}}\right)\right. \\
& \left.\times \frac{(2 \pi)^{4}\left|M_{q \bar{q}, g g \rightarrow t \bar{t}}\right|^{2}}{2 g_{\mu \nu} q_{1}^{\mu} q_{2}^{\nu}} \times W\left(\boldsymbol{y} \mid \boldsymbol{x}, \Delta_{J E S}\right) \times d \Phi(\boldsymbol{x})\right] .
\end{aligned}
$$




\subsection{Background Probability}

The probability for $W+$ jets production, $P_{W j e t s}$, can be calculated in a similar way as the signal probability $P_{s i g}$ by replacing the $t \bar{t}$ matrix element $M_{q \bar{q}, g g \rightarrow t \bar{t}}$ by a $W+$ jets matrix element $M_{q \bar{q} \rightarrow W j e t s}$ :

$$
\begin{aligned}
P_{W j e t s}\left(\boldsymbol{y} \mid \Delta_{J E S}\right) & =\frac{1}{\sigma_{p \bar{p} \rightarrow W+j e t s, \text { obs }\left(\Delta_{J E S}\right)}} \times \sum_{\begin{array}{c}
\text { quark } \\
\text { flavours }
\end{array}} \int\left[d \boldsymbol{q}_{\mathbf{1}} d \boldsymbol{q}_{\mathbf{2}} f\left(\boldsymbol{q}_{\mathbf{1}}\right) f\left(\boldsymbol{q}_{\mathbf{2}}\right)\right. \\
& \left.\times \frac{(2 \pi)^{4}\left|M_{q \bar{q} \rightarrow W j e t s}\right|^{2}}{2 g_{\mu \nu} q_{1}^{\mu} q_{2}^{\nu}} \times W\left(\boldsymbol{y} \mid \boldsymbol{x}, \Delta_{J E S}\right) \times d \Phi(\boldsymbol{x})\right]
\end{aligned}
$$

The fact that we involve just the $W+$ jets processes in the calculation of the likelihood $L\left(\boldsymbol{Y} \mid m_{t}, \Delta_{J E S}\right)$, excluding all the other backgrounds, reflects an incompleteness of the model. However the method works well with a small contamination by events not described by the model. An incomplete background likelihood, indeed, will lead to a shift of the measured top quark mass value (apart from an increased statistical uncertainty); the shift will in general depend on the top quark mass itself and on the fraction of events in the sample that are not accounted for in the overall likelihood. The shift is determined in the final calibration procedure in which we will map the $m_{t}$ values into the MC mass inputs, and the uncertainties are obtained by studying pseudo-experiments. When a background term is omitted in the event likelihood, the situation will not be qualitatively different from that in an analysis that includes this term in the likelihood [38.

In the following sections we are going to explain in more detail the main factors of the signal and background probability expressions.

\subsection{Matrix Element}

The matrix element is, obviously, the fundamental part of the method used in this analysis. For signal production, it is the amplitude for production and decay of heavy $t \bar{t}$ pairs in hadronic collisions. We use the matrix element expression developed by Kleiss and Stirling [68 which includes both the process of annihilation $q \bar{q} \rightarrow t \bar{t}$ and the gluon-gluon fusion $g g \rightarrow t \bar{t}$, as well as the complete spin correlations in the decay $t \bar{t} \rightarrow b(l \nu) \bar{b}\left(q \bar{q}^{\prime}\right)$. 
As far as the matrix element for $W+$ jets production is concerned, there is a fundamental difference with respect to the signal matrix element: even at leading order, hundreds of Feynman graphs are needed to describe electroweak $W+$ jets production, leading to more complicated (and expensive in terms of processing time) algorithms employed in the computation. The $W+$ jets matrix element is calculated at leading order as implemented in MADGRAPH 5 program 45. MADGRAPH is a tool for automatically generating tree-level matrix elements at given phase space points for High Energy Physics processes. Before employing MADGRAPH, we have been using the VECBOS program [36] in developing the matrix element for $W+$ jets production. We decided to switch to MADGRAPH, due to some problems in VECBOS related to the convergence of the integration and compiler, as described in the next chapter.

\subsection{Parton Distribution Functions}

The Parton Distribution Functions $f\left(\boldsymbol{q}_{\boldsymbol{i}}\right)$ of the proton (antiproton) are a necessary input to almost all theory predictions for hadron colliders. The parton density function gives the probability of finding in the (anti)proton a parton $i$ with a specific flavor carrying a fraction $\boldsymbol{q}_{\boldsymbol{i}}$ of the proton momentum. Cross section are calculated by convoluting the parton level cross section with the appropriate PDFs. We use the CTEQ5L leading order PDFs [42], with appropriate weights to account for $q, \bar{q}$ and $g$ contributions.

\subsection{Jet Energy Scale Parameter}

The jets originating from the fragmentation of quarks and gluons are the most common and complicated final state objects produced at hadron colliders. Several correction factors are developed to estimate the original parton energy from the observed jet energy in the calorimeter. The CDF jet energy corrections are divided into different levels to accommodate different effects that can distort the measured jet energy, as different response of the calorimeter, non-linear calorimeter response to the deposited energy, un-instrumented regions of the detector, energy released into the jet cone from overlapping (spectator) interactions, and jet energy radiated outside the pre-defined jet cone. According to these corrections, the jet energy scale parameter $(J E S)$ relates the transverse momentum of a jet observed inside the jet cone, $p_{T, j e t}$, to the transverse momentum of the contained particles, 
$p_{T, M C}:$

$$
p_{T, M C}=J E S \cdot p_{T, j e t}{ }^{1}
$$

Several significant sources of uncertainties are introduced on the jet energy scale determination. Since the lepton + jets signature contains at least four jets in the final state and our measurement technique makes explicit use of the jet transverse momenta, imperfect calibration and corrections of the detector jet energy scale would result in a potentially large source of uncertainty in our measurement of the top quark mass. Therefore, instead of converting the uncertainty of the jet energy scale in our top mass measurement by the common way, i.e. shifting the jets by their uncertainty and measuring the resulting difference in the top mass, we calibrate the jet energy scale in situ making use of the fact that the hadronically decaying $\mathrm{W}$ boson daughters should form a final state whose invariant mass is consistent with the known $\mathrm{W}$ boson mass and width. The constraint of $M_{W}=80.4 \mathrm{GeV}$ is imposed by integrating over $W$ boson masses using a Breit-Wigner prior. This allows us to constrain the jet energy scale and convert the JES systematic uncertainty into a smaller statistical uncertainty. We describe the likelihood as a function of $\Delta_{J E S}$ and top mass parameters. The common $\Delta_{J E S}$ for all jets (determined from data) automatically tunes the likelihood to reproduce the $\mathrm{W}$ peak correctly, as there is a strong peak in the $t \bar{t}$ matrix element due to the $\mathrm{W}$ propagator.

In our top mass measurement, JES is related to the fractional systematic uncertainty from prior calibration, $\sigma_{j e t}$, by

$$
J E S=1+\Delta_{J E S} \cdot \sigma_{J E S}\left(p_{T, j e t}\right)
$$

where $\Delta_{J E S}$ is a $p_{T}$-independent nuisance parameter and $\sigma_{J E S}\left(p_{T, j e t}\right)$ is the $p_{T}$-dependent systematic uncertainty. For instance, $\Delta_{J E S}=1$ means that all jets are shifted upwards by $1 \sigma$, an amount that will be different for jets of different energy.

\subsection{Transfer Functions}

The transfer functions $W\left(\boldsymbol{y} \mid \boldsymbol{x}, \Delta_{J E S}\right)$ are one of the most important component of any matrix element analysis, as they connect the parton-level quantities $\boldsymbol{x}$ appearing in the matrix element with the reconstructed quantities

\footnotetext{
${ }^{1}$ This is inverse to the standard $J E S$ convention used by CDF publications. We shall stick to it during the analysis and return to the standard convention when computing the final likelihood.
} 
observed in our detector. The total transfer function used in the probability calculation in Eq.5.9 can be in general split up into a product of single transfer functions for each object observed by detector. However, we only apply our transfer functions to the jets because we assume that the lepton momentum is well measured in our detector (in these circumstances the transfer function for lepton momentum is described by a delta function). Also the $\mathbb{E}_{T}$ related to neutrino is not used in defining event probabilities, since it does not carry sufficient information. This is the equivalent of setting the transfer function for $\mathbb{E}_{T}$ to the unity.

In developing the jet transfer functions, it is necessary to take into account the ambiguity in assigning measured jets to partons from $t \bar{t}$ decay. Since at LO there are four jets in the final state, all $4 !=24$ jet-parton assignments are considered. The inclusion of $b$-tagging information provides improved identification of the correct jet-parton assignments through appropriate weights $w_{i}$. In addition we factorize the transfer function into separate momentum and angular parts as follows:

$$
W\left(\boldsymbol{y} \mid \boldsymbol{x}, \Delta_{J E S}\right)=\sum_{i=1}^{24} w_{i}\left[W\left(p_{t, j e t} \mid p_{t, p a r t}\right) W\left(\eta_{j e t}, \phi_{j e t} \mid \eta_{\text {part }}, \phi_{\text {part }}\right)\right],
$$

where jet subscripts indicate the jet quantities and the part subscripts the parton quantities. This is important, because the response of the detector to jets is different at different polar angles, and so are the transfer functions.

Starting from MC events, we derive the transfer functions, matching the jets to the partons by means of algorithms that correlate the direction of the primary to the jet axis, and then building the distributions. The angular transfer function is built as the probability distribution of $\Delta \eta$ and $\Delta \phi$, the differences between the $\eta$ and $\phi$ of the jet and the parton. A sample angular transfer function is shown in Figure 5.1 61.

For the momentum transfer function, basically, we need to solve Eq. 5.11 for unknown quantity $p_{T, j e t}$, making use of the definition in Eq. 5.12. Once obtained $p_{T, j e t}$ from the known quantities $p_{T, M C}$ and $\Delta_{J E S}$, we can build the distribution of $p_{T}$ ratio $u=\frac{p_{T, j e t}}{p_{T, p a r t}}$, where $p_{T, \text { part }}$ is the transverse momentum of the parton that initiates the corresponding $\mathrm{MC}$ jet. In Figure 5.2 an example of momentum transfer function is shown 61]. 


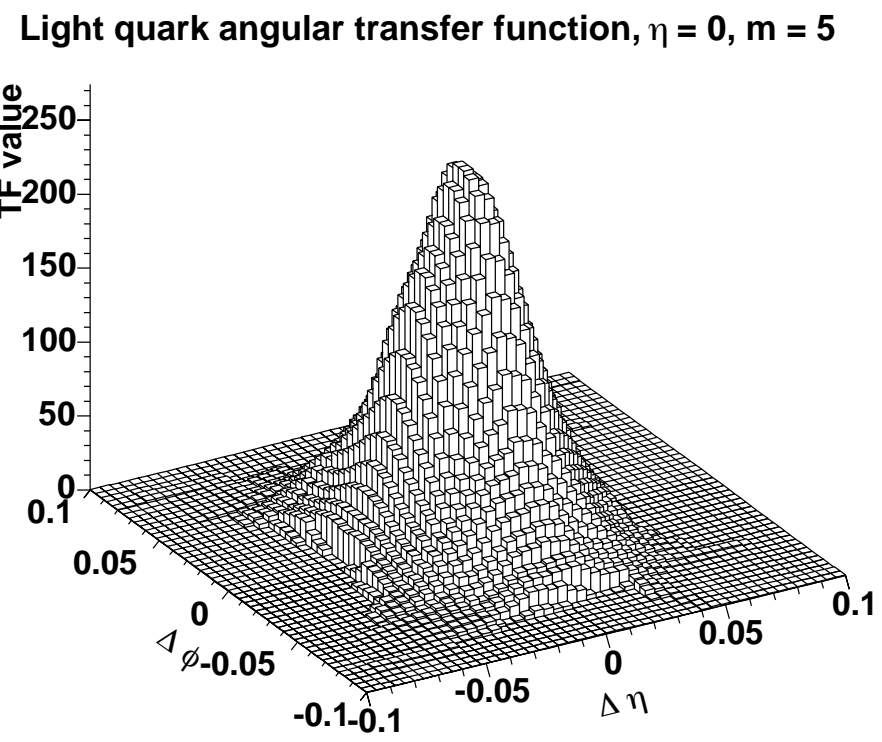

Figure 5.1: Sample angular transfer function as a function of $\Delta \eta$ and $\Delta \phi$ (in radians) [61.

\subsection{Normalization}

Equations 5.8 and 5.9 show that the normalization of the signal probability is guaranteed by the inclusion of the total $t \bar{t}$ production cross section along with the acceptance factor. The integral of the product between these terms, defining $\sigma_{p \bar{p} \rightarrow t \bar{t}, a c c}$ (Equation 5.8), represents an approximation of the cross section observed in the detector, which is practically impossible to compute in advance. In this sense, the acceptance that we use in the definition of the likelihood is not the conventional acceptance used in cross section measurements.

The $t \bar{t}$ cross section is not constant as a function of $m_{t}$. Therefore we add to the cross section a weight factor $N\left(m_{t}\right)$, which is calculated with a leading order integration over the phase space, including all the components of the probability function (see Eq. 5.9p), except for the transfer function. 


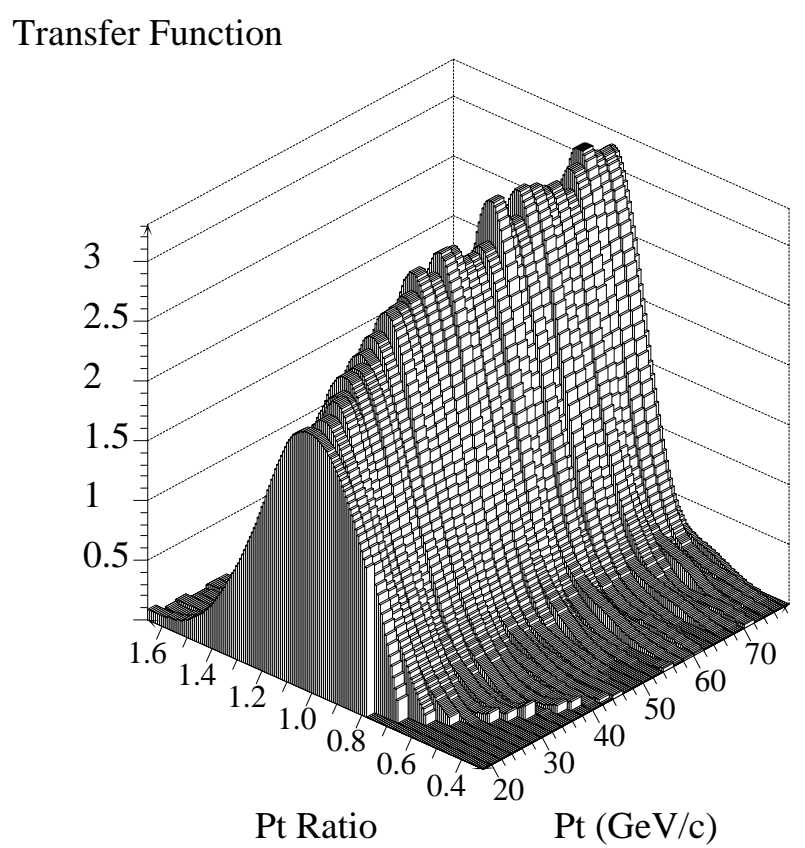

Figure 5.2: Sample momentum transfer function as a function of the $p_{T}$ ratio $u=\frac{p_{T, j e t}}{p_{T, p a r t}}$ and the parton $p_{T}$ [61].

\subsection{Acceptance}

The purpose of calculating the acceptance term $A\left(m_{t}, \Delta_{J E S}\right)$ for the ME integration consists in normalizing the event probability over the observed variables, as a function of $m_{t}$ and $\Delta_{J E S}$, rather than by event counting. For the $t \bar{t}$ acceptance calculation we use MC events generated by PYTHIA in the range of top mass between $155 \mathrm{GeV}$ and $195 \mathrm{GeV}$. We build the acceptance separately for the untagged, single-tagged and double tagged samples, separately for tight and loose categories, as described in Chapter 4.

We define the acceptance as the ratio between the number of selected events in the specific category and the number of the total lepton + jets generated events:

$$
A\left(m_{t}, \Delta_{J E S}\right)=\frac{N_{s e l}}{N_{g e n}} .
$$

Although this is the usual definition of the acceptance, we don't compute it in the conventional way, as we impose conditions such as jet-parton matching, 
usually forbidden in the definition of the conventional acceptance, where the denominator should be fully unbiased. In addition to electron + jets and muon + jets events, the number of generated events considered in the definition of the acceptance includes also $\tau+$ jets events. Both the count for generated events and the count for selected events require matching between the first four jets and the corresponding assigned partons. The algorithm used for the matching is based on a cone method: for each primary parton we search for the closest jet within $\Delta R<0.4$, where $\Delta R=\sqrt{\Delta \eta^{2}+\Delta \phi^{2}}$.

To evaluate the number of selected events we apply to the generated events the selection cuts described in Table 4.1 and the additional requirement of matching between the reconstructed leptons and the generated leptons. Here, we deal separately with each category, taking into account the additional separation between leptons identified as $\tau$ and non- $\tau$ leptons. The reason is that we need to integrate separately the event probability in the two cases because the convergency of the integral is different as well as the uncertainties obtained: consider that the final state observed in the detector due to a $\tau+$ jets event includes at least one additional neutrino and more objects. Therefore the event probability for such events has to be integrated over a wider phase space and consequently the calculation of the related acceptance term is needed.

The acceptance term is then calculated for each category varying $m_{t}$ in the range $155-195 \mathrm{GeV}$ with steps of $1 \mathrm{GeV}$ and $\Delta_{J E S}$ between -3 and +3 with spacing of 0.2 . In Figure 5.3 the acceptance calculated for the untagged tight category is shown.

The value of the acceptance is given in percentage. The relative acceptance error, that is not shown in the current plot, is evaluated by propagating the errors associated to the numerator and denominator defining the acceptance term. It results to be of the order of $\sim 1 \%$.

Note that the acceptance increases as the JES shift decreases. Indeed, according to Eq. 5.12, JES increases with $\Delta_{J E S}$ and consequently, given Eq. 5.11, the detector $P_{T, j e t}$ decreases for fixed $P_{T, M C}$.

In Figure 5.4 some one-dimensional projections of the acceptance for the 0 -tag tight category are illustrated. The first row shows the acceptance for $l+j e t s$ final states with $l \neq \tau$ : the acceptance is calculated as a function of $\Delta_{J E S}$ fixing the top mass value to $172 \mathrm{GeV}$ (top left plot), and as a function of $m_{t}$ with fixed $\Delta_{J E S}=0$ (top right plot). The second row shows the same plots for $l+j$ ets final states with $l=\tau$. The acceptance decreases in the second case because of the cut applied on the final lepton momentum and 


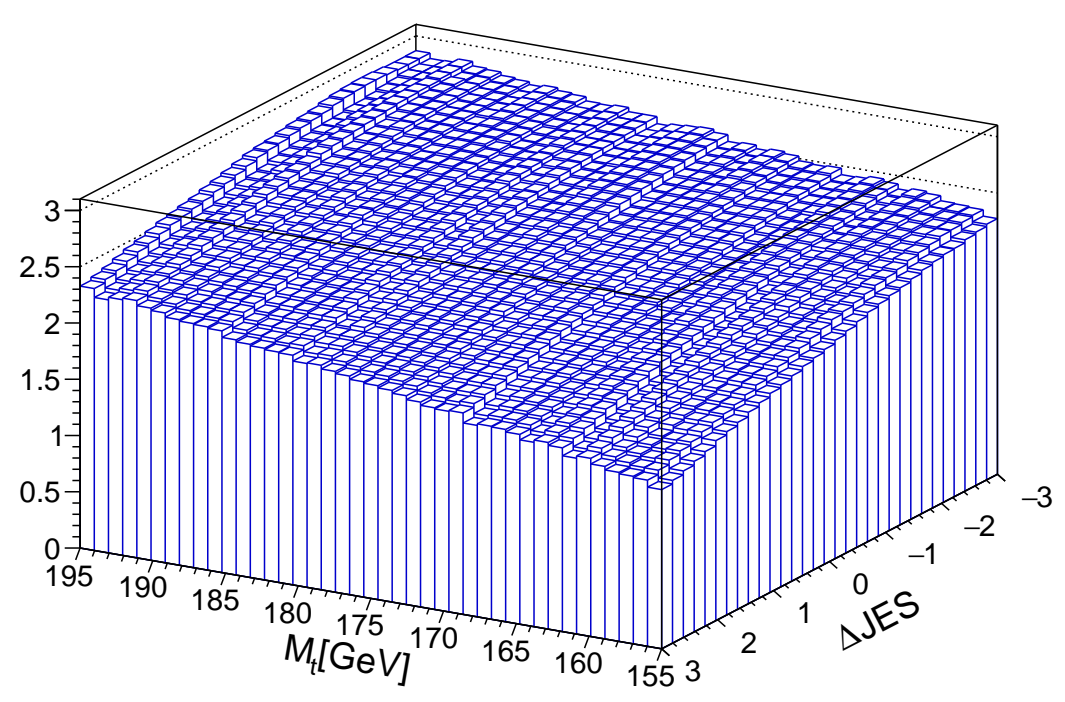

Figure 5.3: Acceptance for 0-tag tight category with $l \neq \tau$ as a function of $m_{t}$ and $\triangle_{J E S}$.

this reduction implies also more fluctuations. What we detect in the case of tau leptons, in fact, is either an electron or a muon coming from the $\tau$ decay, therefore these secondary leptons are in general less energetic than the primary one and a smaller number of them will pass the selection.

Two similar projections of the acceptance evaluated for 2-tag tight category and $l \neq \tau$ are shown in Figure 5.5. Even in this case we notice a reduction of the acceptance value with respect to the 0 -tag case because of the more strict requirement of two $b$-tagged jets. 


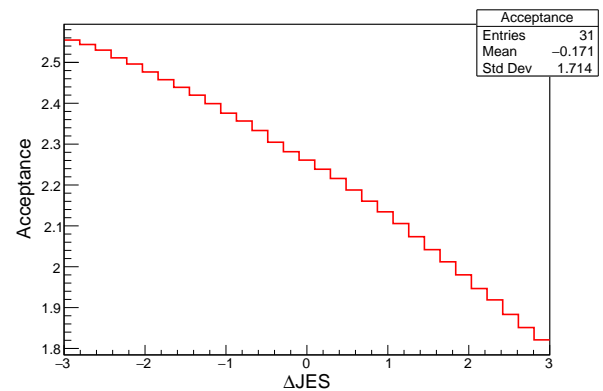

(a) Acceptance for the $l+$ jets sample with $l \neq \tau$ as a function of $\Delta_{J E S}$ with $m_{t}=172 \mathrm{GeV}$.

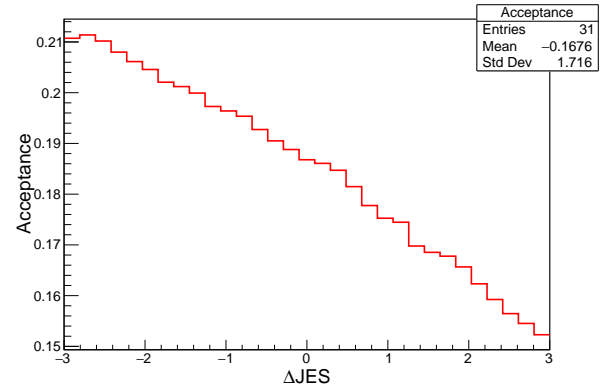

(c) Acceptance for the $l+$ jets sample with $l=\tau$ as a function of $\Delta_{J E S}$ with $m_{t}=172 \mathrm{GeV}$.

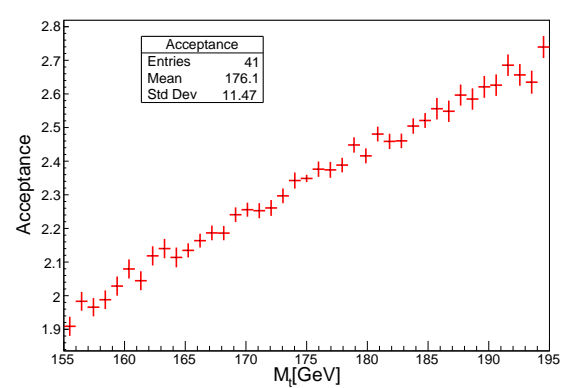

(b) Acceptance for the $l+$ jets sample with $l \neq \tau$ as a function of $m_{t}$ with $\Delta_{J E S}=0$.

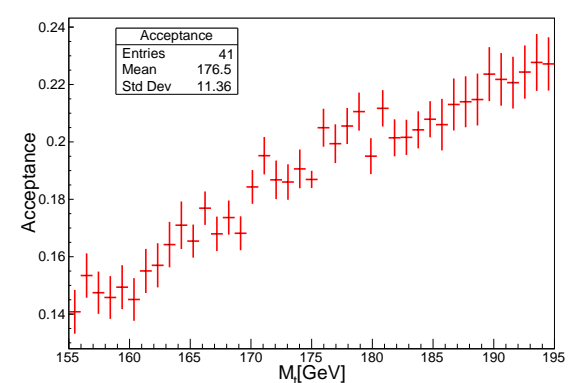

(d) Acceptance for the $l+j$ jets sample with $l=\tau$ as a function of $m_{t}$ with $\Delta_{J E S}=0$.

Figure 5.4: One-dimensional projections of the acceptance for 0-tag tight category. 


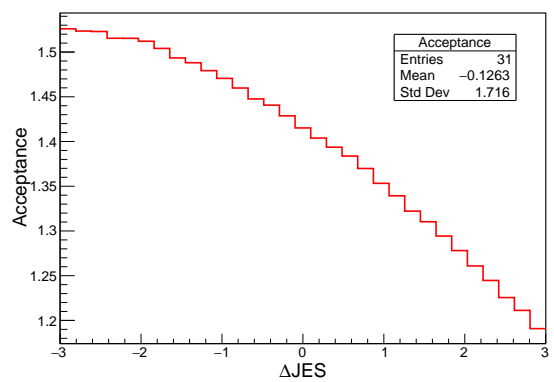

(a) Acceptance for the $l+$ jets sample with $l \neq \tau$ as a function of $\Delta_{J E S}$ with $m_{t}=172 \mathrm{GeV}$.

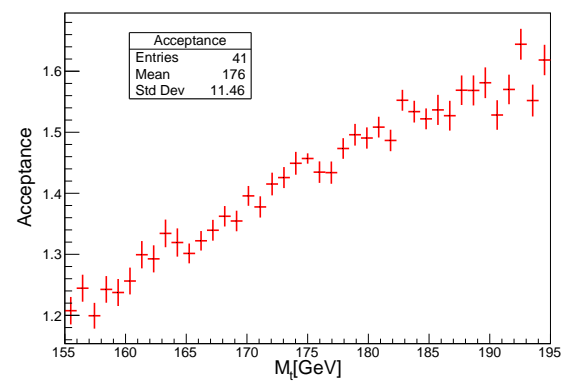

(b) Acceptance for the $l+j$ ets sample with $l \neq \tau$ as a function of $m_{t}$ with $\Delta_{J E S}=0$.

Figure 5.5: One-dimensional projections of the acceptance for 2-tag tight category.

\subsection{Integration variables}

We have 32 variables available to describe the kinematics of a $t \bar{t}$ production and decay, four for each of the two incoming partons and six final state objects: two light quarks, two $b$ quarks, one lepton and one neutrino. The number of independent variables is reduced by introducing some constraints and assumptions. Four of these dimensions are eliminated by energy and momentum conservation, and four more by taking the charged lepton, neutrino, and initial parton masses as known. In addition, we assume that the lepton momentum is perfectly measured, and we neglect the transverse momenta of the initial partons so that we model only the transverse momentum of the total $t \bar{t}$ system. This leaves a total of 19 dimensions over which the integral must be evaluated ${ }^{2}$. We then select the following 19 variables:

- $M_{t, l e p}^{2}$ and $M_{t, h a d}^{2}$, the squares of the masses of the top quarks on the hadronic and leptonic decay channel;

- $M_{W, l e p}^{2}$ and $M_{W, h a d}^{2}$, the squared mass of the leptonic and hadronic $W_{\mathrm{s}}$;

- $\beta=\ln \frac{p_{q}}{p_{\bar{q}}}$, the logarithm of the ratio of the magnitude of the light quark momenta from the hadronic side decay;

- $\vec{p}_{T}(t \bar{t})$, the transverse momentum of the $t \bar{t}$ system;

- $m_{1 \ldots 4}$, the masses of the four partons that initiate the jets in the final state;

\footnotetext{
${ }^{2}$ Note that in the case of $\tau$ lepton decays we have an additional integration dimension due to the charged lepton momentum.
} 
- $\eta_{1 \ldots 4}$ and $\phi_{1 \ldots 4}$, the angles of the four partons that initiate the jets.

After the reduction of dimensions, the integral still remains a complicated work. Moreover, the integrand has a complex structure because of the resonant nature of $t$ and $W$, and its efficient evaluation requires a highly nontrivial phase space sampling scheme. In most of the previous analyses it has been necessary to introduce additional assumption in order to speed up the convergence of the integration procedures. These additional and often imperfect assumptions cause an inevitable loss of resolution in the result. In this analysis we employ an improved integration technique, named QuasiMonte Carlo (Quasi-MC) technique [63], that allows us to integrate over the wide phase space without significantly increasing the computation time required to evaluate the likelihood, resulting in an improved mass resolution.

\subsection{Phase Space}

The integration of the event probability is performed over the differential phase space $d \Phi(\boldsymbol{x})$. According to the Fermi's golden rule the differential phase space of the $t \bar{t} \rightarrow b(l \nu) \bar{b}\left(q \overline{q^{\prime}}\right)$ decay written down in terms of the four-momenta of the particles involved, is proportional to

$$
\prod_{i=1}^{4} \frac{d^{3} \vec{p}_{i} d E_{i}}{(2 \pi)^{3}} \cdot \frac{d^{3} \vec{p}_{l}}{(2 \pi)^{3} 2 E_{l}} \cdot \frac{d^{3} \vec{p}_{\nu}}{(2 \pi)^{3} 2 E_{\nu}}
$$

Here, $\vec{p}_{i}$ and $E_{i}$ represent the three-momentum and energy of the four outgoing quarks of the decay, $l$ and $\nu$ subscripts are referred to the lepton and neutrino respectively. The difference between terms referred to lepton and neutrino, and the one referred to partons comes from the fact that, as previously specified, the lepton and neutrino masses are taken as constants, while the parton masses are allowed to vary in our integral. Moreover, because of our previous assumption that the lepton momentum is well measured, the integration over $p_{l}$ is eliminated. We can also rewrite the three-momenta in terms of their magnitude $p_{i}$ and angles $\Omega_{i}$ :

$$
\prod_{i=1}^{4} \frac{p_{i}^{2} d p_{i} d \Omega_{i} d E_{i}}{(2 \pi)^{3}} \cdot \frac{d^{3} \overrightarrow{p_{\nu}}}{(2 \pi)^{3} 2 E_{\nu}}
$$

At this point, by introducing the Jacobian matrix, the transformation from $p_{1} \ldots p_{4}, \theta_{1} \ldots \theta_{4}, \phi_{1} \ldots \phi_{4}, E_{1} \ldots E_{4}, p_{\nu x}, p_{\nu y}, p_{\nu z}$ into the variables actually used in our integration has to be performed. 
Quasi-MC integration is used for 18 of the 19 variables available. The leptonic $\mathrm{W}$ mass $M_{W, l e p}^{2}$ requires special treatment to avoid phase space singularities. Specifically, when considering the allowed values of the squared leptonic W mass $M_{W, l e p}^{2}$ vs the neutrino z-momentum $p_{\nu z}$, the Jacobian for transforming between these two variables goes to zero at a point, and so, since the Jacobian is in the denominator, the integrand becomes infinite. Similarly, using $p_{\nu z}$ as the integration variable will also fail in some cases. To avoid these problems, the integration code switches between the two integration variables as necessary and the integration is performed over a fixed grid 61.

\subsection{Advantages of the Matrix Element Method}

As already mentioned, the Matrix Element is not the only method used in analyzing collision data for the purpose of measuring top quark mass. In particular, in addition to the ME Method, another fundamental technique has been widely used by the Tevatron experiments in top mass analysis: the template method.

Template Method In the template method, simulated distributions are constructed for a chosen quantity (estimator) sensitive to the physics observable under study, the top mass. Both signal and background distribution are constructed from MC samples, using a number of discrete values of $m_{t}$. Often the jet energy scale is included as the second parameter. These MC derived distributions are called "templates". Any measured quantity in the event that is correlated with the mass of the decaying top quark can be used as estimator in the analysis. One example is the reconstructed top mass with the best $\chi^{2}$ from the kinematic fit $m_{t}^{r e c o}$ as well as the dijet mass from the hadronically decaying $W[9 \mid$. In all cases, it is mandatory to understand at best the top quark mass dependence of the adopted parameter. Then these template distributions are fitted to functions that interpolate between different input values of the physics observable, fixing all other parameters of the functions. In the final step a likelihood fit to the observed data distribution is used to obtain the value for the physics observable that best describes the data.

Both the matrix element and the template technique have been exploited in the top mass measurement in a variety of distinct approaches, with both complementary and competing features. In each approach several issues have to be considered and addressed in a consistent manner. Some of these issues are the choice of the $t \bar{t}$ final state, the event sample selection, the degree to which the method depends on the calibration of the jet energy scale. Also the 
ambiguity of assigning jets to the $t \bar{t}$ decay has to be considered, as well as the problem of choosing a correct set of jets when the number of jets observed exceeds the number of strongly interacting partons produced in the leading order perturbation theory.

Based on these considerations, the most convenient method of analysis is choosen.

ME technique has generally proven to be the most precise method available to measure the top mass. As explained in the previous sections, in the ME method, for each selected event, the likelihood to observe it is calculated as a function of the assumed top quark mass. To this end, all possible reactions yielding final states that could have led to the observed event are considered. An integration is performed over all possible momentum configurations of the final state particles for all relevant reactions. Since the full topology of the event is exploited, the Matrix Element approach offers several important advantages over all other data analysis schemes [74]:

- The theoretical assumptions about the process under study (parton distribution function, matrix element, transfer functions) are incorporated into the data analysis in the most efficient manner. In the limit where all the quantities and functions in the event probability expression are known with perfect accuracy, by the Neyman-Pearson Lemma, the likelihood is an optimal test statistic 22 .

- Some widely used data analysis methods, like the template technique, introduce implicit assumptions about the shape of detector resolution functions. In particular methods based on $\chi^{2}$ minimization (used in kinematic fitting) assume Gaussian measurement errors, and the effect of this assumption on the quality of statistical modeling cannot be quantified within the $\chi^{2}$-based method itself. There is no such inherent restriction on the ME approach; very detailed and precise detector models can be usefully employed.

- Maximization of the likelihood $L(\boldsymbol{a})=\prod P_{e v}\left(\boldsymbol{y}_{i} \mid \boldsymbol{a}\right)$ results in an efficient (in the statistical sense) estimate of the parameter $\boldsymbol{a}$. Straightforward profiling or marginalization of the likelihood can be utilized in case some of the $\boldsymbol{a}$ dimensions are not of interest and can be treated as nuisance parameters. In effect, systematic uncertainties (which are nothing else but the uncertainties due to imprecisely known values of nuisance parameters) are calculated on the event-by-event basis and, therefore, each event contributes into the overall parameter estimate 
with an optimal weight which takes into account both statistical and systematic uncertainty.

Hence, if it can be implemented, the ME method should be the most sensitive analysis possible. Despite all the advantages mentioned, the matrix element approach is not necessarily the obvious first choice among various high energy data analysis techniques. A practical challenge associated with the use of the ME technique is that the likelihood calculated is only an approximation of the true likelihood. This situation arises because of finite detector resolution, corrections to the fixed-order matrix element, and neglected information. Therefore, to properly calibrate the applied method, one is forced to perform a large number of pseudo-experiments which can be very expensive from the standpoint of computing time. 


\section{Chapter 6}

\section{Integral Computation}

\subsection{Integration Framework}

The integration for our analysis is performed through a complex computation framework consisting in three main parts organized in a hierarchy: one director, one or more masters and several workers. The director coordinates the master and the workers. The workers run on the FermiGrid and are responsible for the actual integration. At a start, the worker interrogates the director which directs it to an available master. The master provides the worker with an event to process. The worker executes the integration and sends the event back to the master to be stored. The process is iterated till completion of the project.

A project is a set of files to be run by a single director with a given configuration set for the integration, defining the matrix elements, the transfer functions, the range of the grid to scan over, the convergence target and so on.

The integration for a single event ends when one of the following three conditions is reached:

- the maximum allowed time to process one event;

- the maximum number of integration points;

- the required integration precision.

For the signal probability the integration is performed on a grid consisting of 61 points in $0.1 \sigma$ intervals in $\Delta_{J E S}$ from -3.05 to $3.05 \sigma$, and 40 points in 1 $\mathrm{GeV}$ intervals in $m_{t}$ included in the range $155-195 \mathrm{GeV}$. For the $W+j$ ets we must study only the dependence on $\Delta_{J E S}$, which is done in the same range and step-spacing as for the signal. 


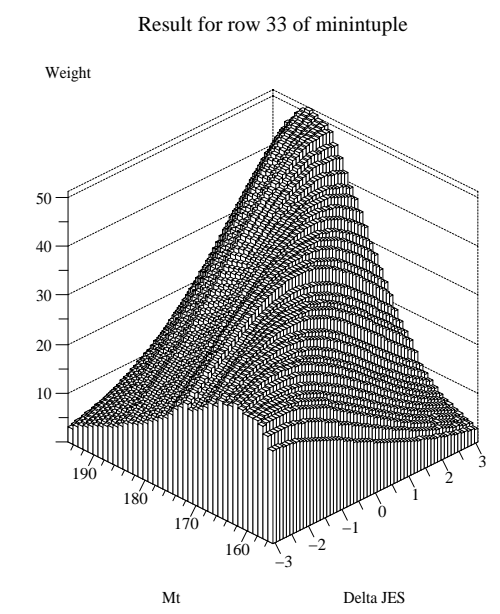

(a) $t \bar{t}$ probability integration output.

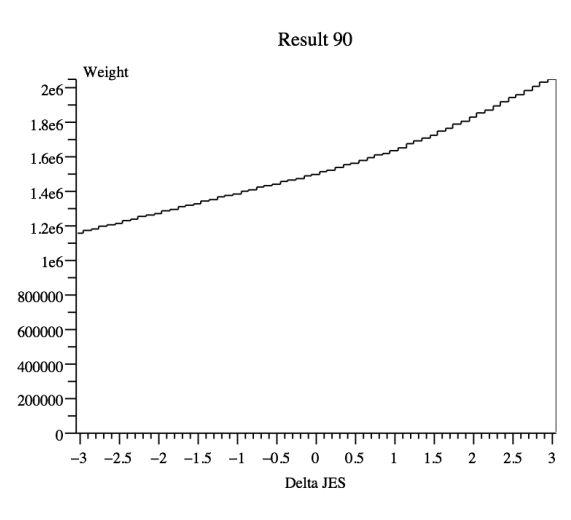

(b) $W+$ jets probability integration output.

Figure 6.1: Examples of output of the two different integrations of signal (a) and background (b).

In Figure 6.1 typical outputs from the two integrations for a signal event and a background event are shown. The bi-dimensional plot on the left shows the output of the $t \bar{t}$ probability integration, the one-dimention plot on the right shows the output of the $W+$ jets integration.

A significant effort was required to devise how to compute the integral with an optimal precision in a reasonable amount of time, as explained in the next section 6.2.

\subsection{Quasi-Monte Carlo Integration}

In our analysis we use Quasi-Monte Carlo (Quasi-MC) integration technique which significantly reduces the time required to integrate an event, allowing us to reduce the number of assumptions made and correspondingly improving the precision and accuracy of our result.

The most common method used for performing integration in physics problem is MC integration, based on sequences of random or pseudo-random numbers. Pseudo-random sequences typically exhibit statistical randomness while being generated by an entirely deterministic causal process. Such a process is easier to produce than a genuinely random one, and has the benefit that it can be used again and again to produce exactly the same numbers. 
Quasi-random sequences are a deterministic alternative to random or pseudo-random sequences for use in MC methods, and it is proved that using such sequences for evaluating multidimensional integrals provides lower error and improved convergence. Quasi-random sequences are generated by choosing points approximately equally spaced in the integration space, such that equal phase space volumes contain approximately equal number of points.

What we can do in general to evaluate the integral of a function $f$ is approximating it with the average of the function evaluated at a set of points $x_{1} \ldots x_{N}$ :

$$
\int_{[0,1]^{s}} f(\vec{x}) \approx \frac{1}{N} \sum_{i=1}^{N} f\left(\overrightarrow{x_{i}}\right) .
$$

Here $N$ is the number of integration points. Since we are integrating over the s-dimensional hypercube $[0,1]^{s}$, each $\vec{x}_{i}$ is a vector of $s$ elements, corresponding to the 18 dimensions ${ }^{1}$ of the phase space in our case. The difference between Quasi-MC and MC is the way the $x_{i}$ are chosen. Pseudo-random sequences used by MC methods are characterized by the property of uniformity, so that contiguous sub-sequences are uniformly spread throughout the cube [63]. However the use of pseudo-random sequences leads naturally to the configuration in which some regions of phase space have more points than average and some regions have fewer points than average. This fact suggests that using more uniformly distributed sequences may produce better results. Therefore Quasi-MC uses low-discrepancy sequences, in which equal subvolumes of the integration space contain as close to equal numbers of points as possible [61]. Figure 6.2 uses two-dimensional projection of a random sequence and of a low-discrepancy sequence to demonstrate the fundamental difference between the two classes of sequences.

Discrepancy is a measure of non-uniformity of the sequence of points placed in the hypercube. Specifically, the most widely used definition of discrepancy is the star discrepancy defined as [4]

$$
D_{N}^{*}\left(x_{1} \ldots x_{N}\right)=\sup _{\substack{0 \leq v_{j} \leq 1, \mid \\ j=1, \ldots, s}}\left|\frac{1}{N} \sum_{i=1}^{N} \prod_{j=1}^{s} 1_{0 \leq x_{i}^{j} \leq 1}-\prod_{j=1}^{s} v_{j}\right|
$$

where $N$ is the number of integration points. In other words, for every subset $E$ of $[0,1]^{s}$, we divide the number of points in $E$ by $N$ and take the absolute difference between this quotient and the volume of $E$. The maximum difference is the star discrepancy $D_{N}^{*}$.

\footnotetext{
${ }^{1}$ We recall that Quasi-MC integration is used for 18 of the 19 variables available, as explained in the previous chapter 5 .
} 


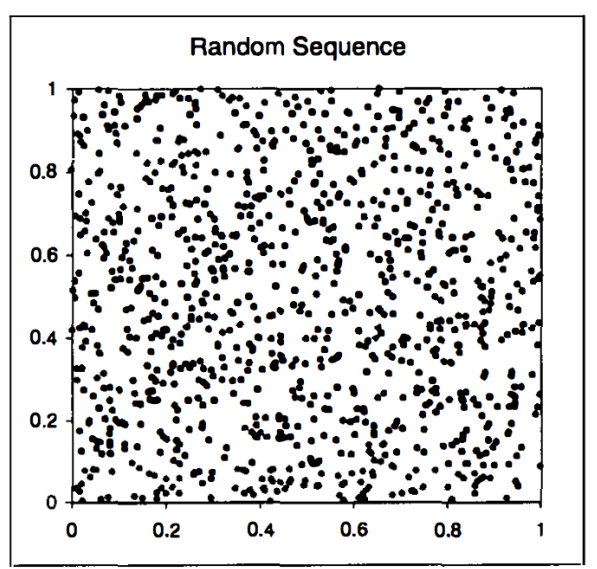

(a)

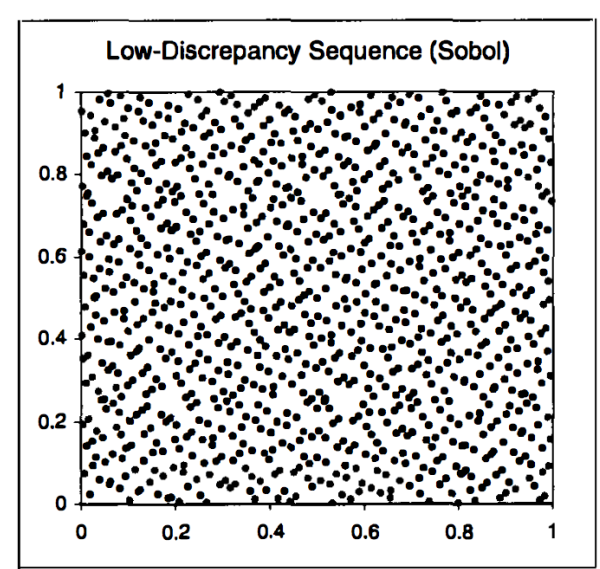

(b)

Figure 6.2: Two-dimensional projection of the first 1024 points in a random sequence (a) and a low-discrepancy Sobol sequence [4].

A sequence $x_{1}, . ., x_{N}$ is a low-discrepancy sequence if, for any $N>1$,

$$
D_{N}^{*}\left(x_{1} \ldots x_{N}\right) \leq c(s) \cdot \frac{(\ln N)^{s}}{N}
$$

where the constant $c(s)$ depends only on the problem dimension $s$. The Koksma-Hlawka inequality states that for Quasi-MC integration of a function $f$ the integration error $\epsilon$ is bounded by

$$
|\epsilon|=\left|\int_{[0,1]^{s}} f(\vec{x})-\frac{1}{N} \sum_{i=1}^{N} f\left(\overrightarrow{x_{i}}\right)\right| \leq D_{N}^{*} V(f)
$$

where $V(f)$ is the variation of the function $f$ 63]. The result is a more rapid convergence of the Quasi-MC integration compared to Pseudo-Monte Carlo (Pseudo-MC). In fact the expected uncertainty of Pseudo-MC integration decreases as $\frac{1}{\sqrt{N}}$, for $N \rightarrow \infty$, whereas the error of the Quasi-MC integration is proportional to $\frac{(\ln N)^{s}}{N}$.

\subsection{Study of the precision of the integration}

Before applying the Quasi-MC method it is necessary to check the precision of a Quasi-MC calculation of the likelihood. This is done by comparing the results with a Pseudo-MC, which provides an undoubtedly sound method. Note that the main property of Pseudo-MC is its robustness. In statistics 
a model is claimed as robust if it still provides insight to a problem despite having its assumptions altered or violated. Pseudo-MC accuracy depends on only the crudest measure of the complexity of the problem. As a function of the number $\mathrm{N}$ of the points in the integration domain, the integration converges at a rate $O\left(N^{-1 / 2}\right)$ that is independent on the dimension of the integral. Basically Pseudo-MC gives an answer that is always qualitatively correct, but the price is its extremely slow convergence. Consequently, using once for always an accurate pseudo-random calculation, we can then switch to Quasi-MC method to study how close the integral becomes to the precise solution calculated with Pseudo-MC, with the advantage of a more rapid convergence. We perform such a study both for the integration of the signal probability and for the $W+$ jets probability separately. This is how the Quasi-MC precision is investigated in the following.

In order to check if the built-in precision determination algorithm of our integrations works correctly with Pseudo-MC we can study the distribution of the pull $\delta_{i}$, defined as the difference between the result $x_{i}$ of the $i^{\text {th }}$ integration and the mean $\mu$ divided by the estimated uncertainty $\sigma_{i}$ :

$$
\delta_{i}=\frac{\left(x_{i}-\mu\right)}{\sigma_{i}}
$$

If a variable $x_{i}$ is generated repeatedly with a Gaussian distribution of mean $\mu$ and width $\sigma_{i}$, the pull will be distributed as a standard Gaussian with mean zero and unit width. Thanks to the central limit theorem, this simple property can be applied in a wide range of situations from hypothesis testing to parameter estimation, where pulls provide evidence for various forms of bias and allow discovering possible errors. In the majority of cases, one expects the pull distribution to be a standard Gaussian. One thus needs to confirm that it is centered at zero, has unit width, and has no long tails. If this is not the case, one may need to look at the measurement setup, the experimenter's assumptions, etc [23]. In order to built such a distribution we run the integration using a large number of integration points.

The pull distribution is obtained by processing the same set of events several times $(\sim 10)$ with different sequence of pseudo-random points, so that they have some statistical spread. We take the results for a specific $\Delta_{J E S}$ value (and top mass value too in the case of $t \bar{t}$ integration) and we use the mean of these results as the "true" value of the integral related with the selected $\Delta_{J E S}$ and $m_{t}$. The difference between a single result and the mean, divided by the estimated uncertainty assigned to the likelihood, that is the 


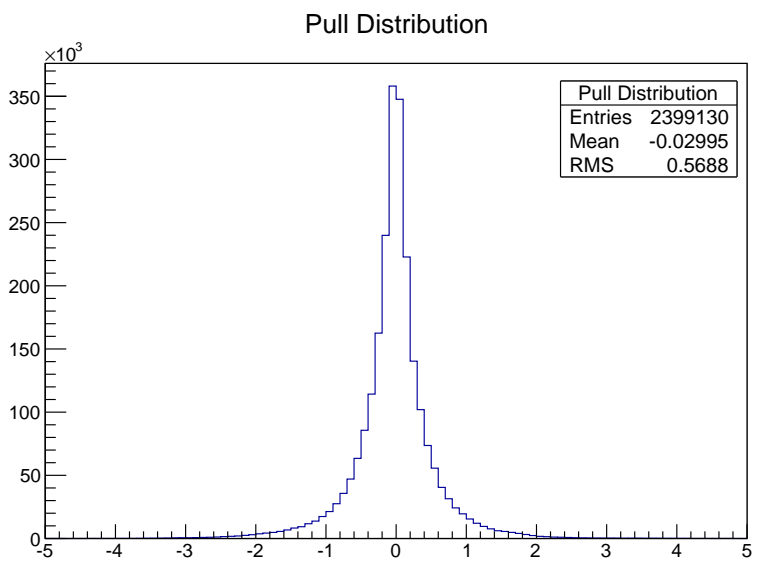

Figure 6.3: Pull distribution related to the $t \bar{t}$ integration.

standard deviation $(R M S) \frac{1}{\sqrt{N}}$, is the needed pull. The pull distribution is built by repeating the procedure for each input value of $\Delta_{J E S}$ and $m_{t}$. In Figure 6.3 is shown the distribution obtained by integrating the $t \bar{t}$ probability in $2^{14}$ points.

With the same procedure we build the pull distribution with the $W+$ jets results. In doing this, we encountered a number of problems that caused a significant amount of extra-work for this thesis work, as described in the following. As can be seen in Figure 6.4 (a), at a first check the pull distribution resulted skewed with a long tail on the left. This fact suggested a problem in our model. We ran again this study with the matrix element calculation (made by VECBOS program [36]) turned off to find that the pull distribution looked more reasonable, as shown in Figure 6.4 (b), even if mildly skewed with a tail on the left, evidence of underestimated errors.

This result proved, as introduced in section 5.4, an evident problem in evaluating $W+$ jets matrix element by using VECBOS, a computing program developed in 1990's with the specific purpose to evaluate fully differential cross sections for the $W+$ jets final state at the Tevatron.

An additional check on this issue is provided by the study of the integral convergence. As explained in section 6.2 the integral of a function $f$ is approximated by the average of the function itself:

$$
\frac{1}{N} \sum_{i=1}^{N} f\left(\overrightarrow{x_{i}}\right) .
$$

We expect to observe this quantity converging to a flat line as the number of integration points $N$ increases. Using matrix element extracted from 


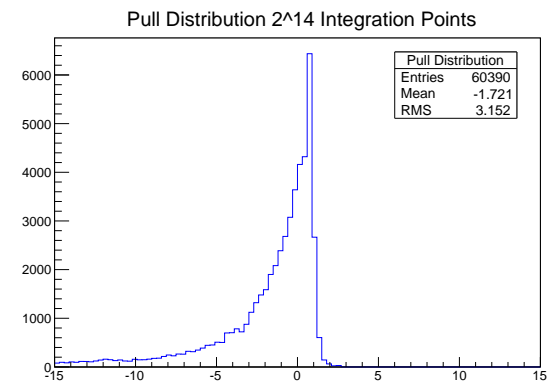

(a) Pull distribution with VECBOS matrix element calculation on.

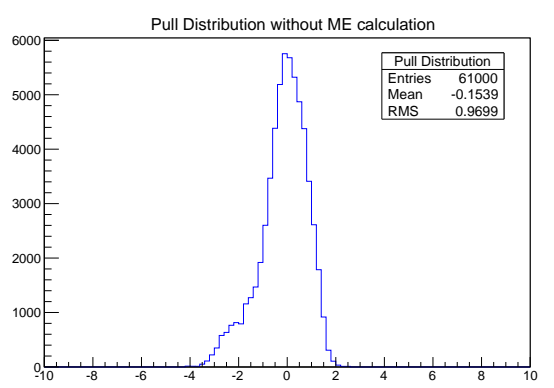

(b) Pull distribution with VECBOs matrix element calculation off.

Figure 6.4: Pull distributions for $W+$ jets integration.

VECBOS, we found instead that for some events the integration was returning diverging values, as shown in Figure 6.5(a).

Analyzing the vECBOS matrix element, we found that the transverse momentum of the $W+4 j e t s$ system was not necessarily null when the momenta of the beam partons were set to zero. This evident violation of four-momentum conservation has been overtaken by boosting the final state particles into the system in which the total transverse momentum of the $W+4 j e t s$ is zero. After fixing the VECBOS matrix element the mean of the integrand looks like a flat line for each event, as shown in Figure 6.5(b).

Nevertheless, after fixing the kinematic problem, a new complication related to VECBOS matrix element appeared: VECBOS produces very different results under different compilers even if the kinematic inputs are the same. Since it was not obvious to understand which compiler, if any, was producing the correct result, we switched to a different matrix element performer, MADGRAPH [45], a more recent framework that aims at providing all the elements necessary for Standard Model phenomenology, such as the matrix element calculation.

The pull distribution built for the integration with the new MADGRAPH matrix element calculation included, is fitted with a Gaussian and reported in Figure 6.6. The result of the fit $($ Mean $=-0.0354$, Sigma $=0.9272)$, attached on the plot, confirms that the distribution is now centered at zero, has a width $\sim 1$ and has regular tails. 


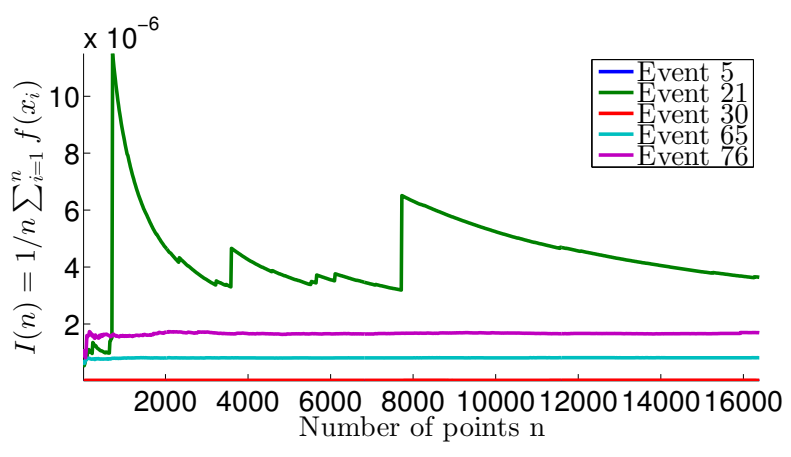

(a) Mean of the integrand before fixing VECBOs matrix element.

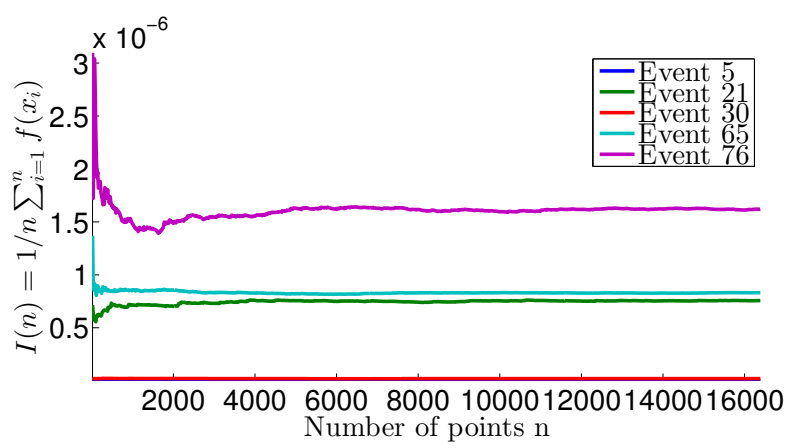

(b) Mean of the integrand after fixing vecBos matrix element.

Figure 6.5: Mean of the integrand as a function of the number of integration points. 


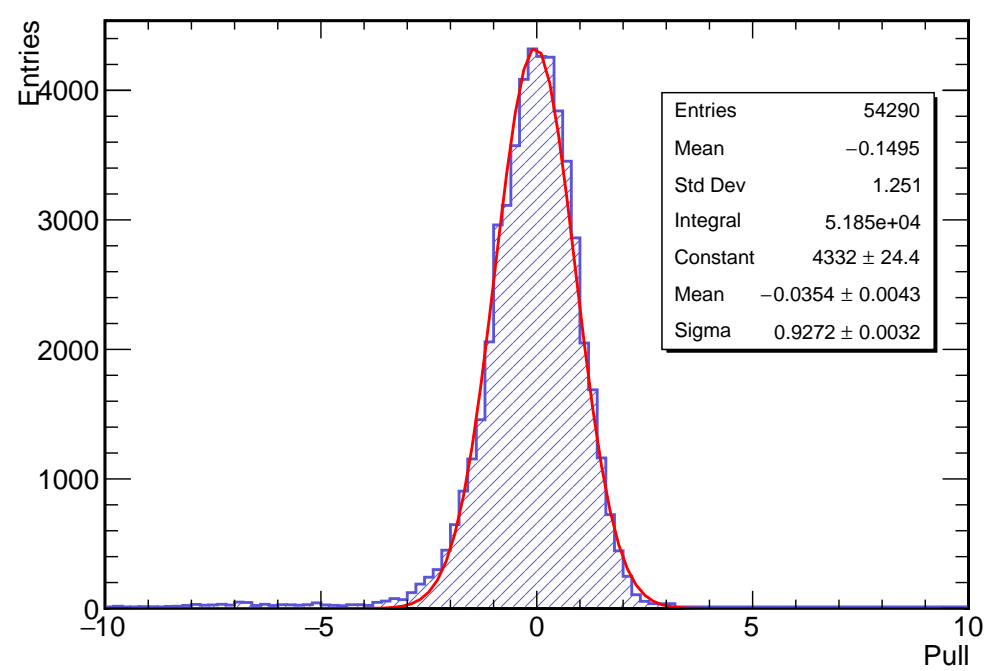

Figure 6.6: Pull distribution related to the $W+$ jets integration with the MADGRAPH matrix element.

\subsection{Calibration of Quasi-MC error}

As mentioned in sections 6.2, the accuracy of the Quasi-MC method increases faster with computing time than that of the (Pseudo-)MC method. However the error associated to the Quasi-MC integration is not known. What we only know is an upper bound on the error, defined by the Koksma-Hlawka inequality in Equation 6.4, i.e. $|\epsilon| \leq D_{N}^{*} V(f)$. However it is not easy to evaluate this quantity since it involves computing $V(f)$, which is typically defined in terms of integrals of partial derivatives of $f$. We determine the error bound as the product between a discrete Fast Fourier Transform of the integrand and a fudge factor to be calibrated. For a complete treatment on the subject see [56]. The calibration of the error bound is a fundamental step for the purpose of our analysis as the integration is terminated when the desired tolerance is reached. The required error bound determines the minimum number of integration points to be used in the computation.

For a given function $f$, if we define our estimator $\hat{I}_{n}(f)$ using $n$ data points, one has:

$$
\hat{I}_{n}(f)=\mu+\epsilon(n),
$$

where $\mu=I(f)$ is the expectation of $f$, and $\lim _{n \rightarrow \infty} \epsilon(n)=0$. If we have 
an upper bound on the error $|\epsilon(n)| \leq \varepsilon(n)$, then it is true that

$$
\left|\hat{I}_{2 n}(f)-\hat{I}_{n}(f)\right|=|\epsilon(2 n)-\epsilon(n)| \leq \varepsilon(2 n)-\varepsilon(n) .
$$

Thus, one first check in calibrating the error bound is ensuring that inequality 6.8 is satisfied within a certain percentage. Otherwise, error bounds are underestimated.

In addition, our interest is to have error bounds that decrease as fast as the real error. Otherwise, if the error bound decreases slower, we would be too conservative or, in the opposite case where the error bound decreases faster, with $n$ large enough, one would find $|\varepsilon(n)| \leq \epsilon(n)$, which would be wrong by definition. Assuming that the real error $|\epsilon(n)|$ is of the form of $C n^{-\alpha}$, we can estimate the value of $\alpha$ doing a regression on the quantity $\left|\hat{I}_{2 n}(f)-\hat{I}_{n}(f)\right|$. If we force $\varepsilon(n)$ to have the same convergence rate as the real error, we should see that $\left|\hat{I}_{2 n}(f)-\hat{I}_{n}(f)\right|$ and $\varepsilon(2 n)-\varepsilon(n)$ share the same exponential convergence rate. 69

In order to calibrate the Quasi-MC error bound we need to tune some parameters in such a way that the main requests are satisfied. Figure 6.7 shows an example on how the calibration looks like for a case where we know the exact solution and integration error (this is not the real case). The blue solid line represents the real error of the integration, whereas the red solid is the error bound to be calibrated. Blue stars indicate $\left|\hat{I}_{2 n}(f)-\hat{I}_{n}(f)\right|$, the quantities that we actually can evaluate, and the red stars represent $\varepsilon(2 n)-\varepsilon(n)$, that is what we can measure with our error bounds and that can be compared to the blue stars. Dashed lines are the regressed lines for the real errors and error bounds and $\alpha$ values are the estimated convergence rates found doing a regression of the form $\mathrm{Cn}^{-\alpha}$. Note that for a Pseudo-MC integration $\alpha=-0.5$. To verify the inequality 6.8 , the red lines should always be above the blue lines. A correct error bound requires in addition that the slopes of the solid lines are the same.

The plots shown in Figure 6.7 are derived from a 10-dimensional quadratic "toy" integrand function. The top plot shows a wrongly calibrated error estimation, the bottom plot represents how the correctly calibrated error bound should appear. In the real case we do not know anything about the real error (blue solid line), so that in the calibration we have to use the estimates represented by the stars in Figure 6.7. We can thus impose the calibration requirements on these quantities: red stars should be above blue stars and the two quantities should have similar convergence rates. 


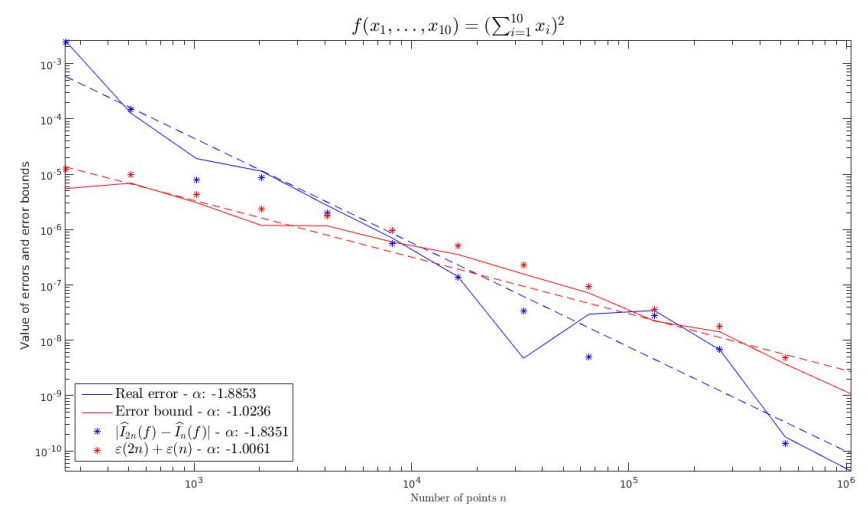

(a) Example of a wrong calibration of the error bound. (The significance of the symbols is explained in the text).

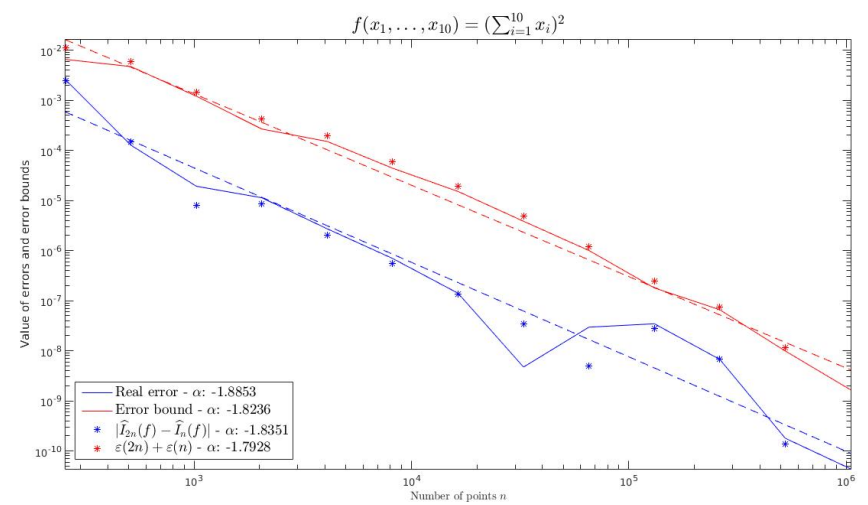

(b) Example of a correct calibration of the error bound. (The significance of the symbols is explained in the text).

Figure 6.7: Calibration of the Quasi-MC error bound.

Depending on the required tolerance, inequality 6.8 might not be satisfied for the considered number of integration points. The more stricht our requirements are, the more expensive we are in terms of number of integration points needed in the calculation and consequently in terms of computation time.

\subsection{Optimization of the integration process}

In principle we could require any high accuracy in the integration, but imposing an excessively strict target precision would be prohibitively timeconsuming. In fact the number of points used in the integration increases with the precision required. Moreover the execution time of the integration 
appears to be proportional to the integration grid size, as it is intuitively expected. The main parameters that define the grid size are the following:

- The number of integration points to use in the integration process.

- The number of points in the leptonic $W$ mass grid. As explained in chapter 5, the $W$ mass grid is separated from the rest of the phase space due to the presence of singularities (i.e., the relevant Jacobian in the integrand denominator has a zero).

- The number of values of $\Delta_{J E S}$ parameter for which the likelihood should be evaluated.

By varying the main parameters separately we can study how the timing of the integration changes. This study can be done both for Pseudo-MC integration and for Quasi-MC one, to see how much is the gain in using Quasi-MC method and to finally tune the optimal configuration in terms of precision, complexity (number of points) and time of the computation.

In Figure 6.8 is shown the variation of the processing time per event as a function of the different grid parameters, in $t \bar{t}$ signal probability integration, calculated with the Pseudo-MC.

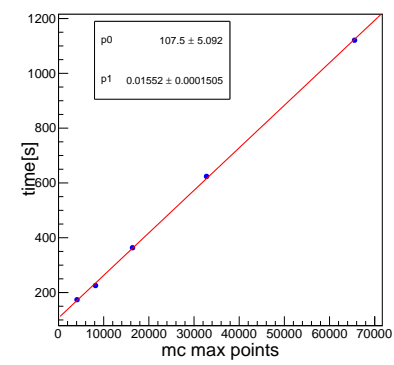

(a) Processing time vs number of integration points.

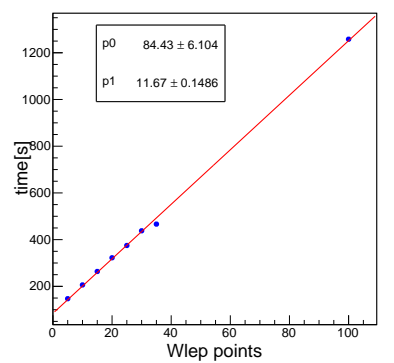

(b) Processing time vs number of points in the $W$ mass grid.

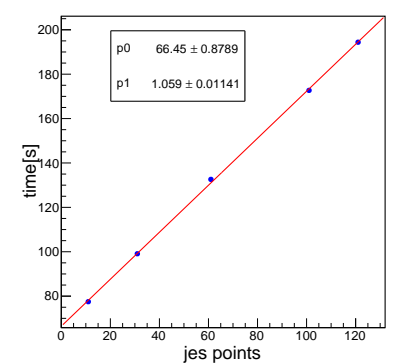

(c) Processing time vs number of $\Delta_{J E S}$ steps.

Figure 6.8: Study of the speed of the integration as a function of grid size parameters.

These plots are obtained by varying one parameter at a time. The plot on the left side is obtained by fixing the number of leptonic $W$ mass grid to 10 (and the number of $\Delta_{J E S}$ set to 31 ) and the one in the middle by fixing the MC points to 1024 (number of $\Delta_{J E S}$ to 31 ). For the plot on the right side the number of $\Delta_{J E S}$ steps changes whereas the other parameters are fixed as in the previous cases. From this preliminary study we can conclude that 
the execution time per event is proportional to the product of the number of MC points and the number of $W$ mass scan points and exhibits only a mild dependence on the number of $\Delta_{J E S}$ steps.

To get some idea on the CPU load, i.e. how expensive integrating one event is, if we suppose to run the integration with $\sim 1000 \mathrm{MC}$ points, $\sim 10$ $W$ mass scan points and $31 \Delta_{J E S}$ points, the time required to integrate a single event with the Pseudo-MC $t \bar{t}$ integration is $\sim 100$ seconds/event.

The same study should be made for the $W+$ jets integration and then for the final likelihood integration which will include both the signal and background probability calculation. A similar study for the Quasi-MC integration should prove how all-together the convergence time is improved over Quasi-MC by using Pseudo-MC.

Making use of these preliminary studies, we will eventually find a tradeoff between the precision desired for the likelihood determination and the required computational time. 


\section{Chapter 7}

\section{Next Steps}

\subsection{Top Mass Extraction}

To obtain the measurement of the top mass $m_{t}$ and the jet energy scale shift $\Delta_{J E S}$, we need to calculate the signal+background combined likelihood for each data event passing the selection requirements described in section 4.2. Given the ensemble of events, the total log-likelihood is then computed by summing the log-likelihoods of the individual events:

$$
\ln L\left(\boldsymbol{Y} \mid m_{t}, \Delta_{J E S}\right)=\sum_{i=1}^{N} \ln P_{e v}\left(\boldsymbol{y}_{i} \mid m_{t}, \Delta_{J E S}\right) .
$$

The maximum (minimum, in case of negative likelihood) of the 2-dimensional likelihood returns the simultaneous measurement of the top mass and JES. From the JES result we can verify how consistent the top mass measurement is with the standard CDF JES. This is essential to know in order to validate, with some tolerance, the systematic uncertainties of the top mass measurement. If the resulting $J E S$ is consistent with the standard CDF jet energy scale, in order to yield a top mass measurement with higher statistical precision, we can consider $\Delta_{J E S}$ as a nuisance parameter that should be eliminated. Nuisance parameters can be usually eliminated either by profiling or by marginalization.

The profiling procedure consists in maximizing the likelihood with respect to the nuisance parameter for each given value of the parameter of interest. In other words $L\left(\boldsymbol{Y} \mid m_{t}, \Delta_{J E S}\right)$ is maximized over $\Delta_{J E S}$ for each value of $m_{t}$, generating the profile likelihood $L_{p}\left(m_{t}\right)$.

Marginalization is instead a Bayesian method that requires introducing a prior distribution $\pi\left(\Delta_{J E S}\right)$ for the nuisance parameter which takes into account 
its uncertainty. Then we obtain the marginalized likelihood by integrating the full likelihood over the prior: $L_{m}\left(m_{t}\right)=\int L\left(m_{t}, \Delta_{J E S}\right) \pi\left(\Delta_{J E S}\right) d \Delta_{J E S}$.

There are certain merits to either approach, and both are common in scientific literature.

After reducing the 2-dimensional curve to a unidimensional likelihood, we can then extract the measured mass and uncertainty from this curve. The measured mass $m_{\text {meas }}$ is simply determined by the position of the peak of the likelihood curve, while the uncertainty is determined by the standard technique of descending one-half unit of log-likelihood from this peak.

As a final step, we can determine $\Delta_{J E S}$ parameter, in order to reduce the systematic uncertainty on the jet energy scale. We can perform the $\Delta_{J E S}$ measurement in exactly the same way of the top mass, eliminating $m_{t}$ using either profile or marginalization method and extracting a measured $\Delta_{J E S}$ and uncertainty from the peak of the profiling or marginalized likelihood.

In measurements which use in situ $J E S$ calibration, the top mass result is always correlated to the JES: $m_{t}$ and $\Delta_{J E S}$ should be reported together and the statistical uncertainty on the top mass should be reported as "statistical $+J E S$ " uncertainty.

\subsection{Validation with Pseudo-Experiments}

Before we can perform a measurement on the data, it is necessary to test our technique on samples with known top masses so that we can verify that it returns correct top mass values and uncertainties for those values. If this is not the case, we have to calibrate the method to appropriately correct these measured values. Several tests must performed with simulated events generated under the assumptions used in the ME method, i.e. using the same PDF set, matrix element, and transfer function.

A pseudo-experiment (PE) emulates a measurement performed on data and consists of events drawn from $\mathrm{MC}$ samples for signal and background processes with known values of $m_{t}$ and $\Delta_{J E S}$ [38. The contribution of each sample to the total has to reflect its expected contribution described in section 4.5. An ensemble of several PEs is performed for each input $m_{t}$ and $\Delta_{J E S}$ value. The range of assumed values is chosen in a wide interval around the expected values of the parameters: we vary $m_{t}$ in the range $155-195 \mathrm{GeV}$ with steps of $1 \mathrm{GeV}$ and $\Delta_{J E S}$ between -3 and +3 with spacing of 0.2 . For a given top mass and $\Delta_{J E S}$, we need to perform $\sim 1000$ PEs. The number 
of $\mathrm{MC}$ events available in our sample is not infinitely large and we may run out of MC events distinct between PEs. This problem can be overcome by using again the same events according to a random shuffling method called bootstrap, which guarantees that the PEs remain statistically independent, although using the same physics information from the MC [2].

For a single $\mathrm{PE}$ we are going to extract a measured mass and uncertainty. Taking the results from all ensembles, the following informations are obtained:

- The relation between the expected (mean) measurement values and the corresponding true input values, i.e. the bias defined as $\left\langle m_{\text {meas }}\right\rangle-m_{\text {true }}$. It is expected that the method yields unbiased results if the ME method reflects the properties of the events.

- The distribution of measurement uncertainties as a function of input parameter values.

- To test that the measured uncertainties describe the actual measurement uncertainty, the deviation of the measurement value $m_{\text {meas }}$ from the true value $m_{\text {true }}$ is divided by the measured uncertainty $\sigma$ in each PE. This deviation defines the pull:

$$
\text { pull }=\frac{m_{\text {meas }}-m_{\text {true }}}{\sigma} .
$$

The standard deviation (RMS) of the distribution of these deviations is referred to as pull width $w$.

A perfect analysis, for which all features of the events are accommodated in the method, would have a bias of 0 , indicating that it correctly measures the input mass, and $w=1$, indicating that it correctly estimates its own uncertainties. However, imperfections in the analysis mean that these conditions are not exactly satisfied. By measuring the bias and pull width on $\mathrm{MC}$ samples, however, we can use these measured quantities to calibrate our data analysis so that we can correctly measure the top mass and its uncertainty.

The necessary steps for the calibration of our top mass analysis are briefly described below:

- Using pure $t \bar{t} \mathrm{MC}$ samples with different top masses we select events in which the four leading jets are matched to quarks produced in $t \bar{t}$ decays. On these samples we should get the slope of the reconstructed top mass as a function of input $m_{t}$ close to 1 and pull width close to 1 as well. Also, we can change JES and reconstruct it back. This might require adjusting the $t \bar{t}$ cross section and efficiency functions. 
- We have to check that we can reconstruct JES correctly from $W+j e t s$ events by changing $\Delta_{J E S}$ in MC. This verifies $W+j e t s$ transfer functions and efficiency as a function of $J E S$.

- We need also to calibrate the relative coefficient for the $W+$ jets density. If the density for $t \bar{t}$ is $P_{s i g}\left(\boldsymbol{y} \mid m_{t}, \Delta_{J E S}\right)$ and the density for $W+$ jets is $P_{W j e t s}\left(\boldsymbol{y} \mid \Delta_{J E S}\right)$, then we need to determine the coefficient $c$ in equation:

$$
P_{e v}\left(\boldsymbol{y} \mid m_{t}, \Delta_{J E S}\right)=f \cdot P_{s i g}\left(\boldsymbol{y} \mid m_{t}, \Delta_{J E S}\right)+(1-f) \cdot c \cdot P_{W j e t s}\left(\boldsymbol{y} \mid \Delta_{J E S}\right),
$$

where $f$ is the fraction of $t \bar{t}$ events in the sample. We can approach this calibration by using a sample which consists only of "matched" $t \bar{t}$ events and $W+$ jets. For this sample, we should be able to reconstruct correctly both input $m_{t}$ and $f$. It should be sufficient to perform this calibration only for $\Delta_{J E S}=0$.

- Finally we deal with the construction of the full $m_{t}$ and $J E S$ mapping function using the complete $t \bar{t}$ and background $\mathrm{MC}$ for a number of input $m_{t}$ and $\Delta_{J E S}$ values.

\subsection{Systematic Uncertainties}

For the measurement, the uncertainties in the properties of the full simulation used for the calibration have to be accounted for by systematic uncertainties on the measurement result. Furthermore, any defect of the MC simulations used to setup the method, cannot be caught by the calibration. Therefore we have to take into account several sources of uncertainty.

Systematic uncertainties arise, indeed, from three sources: modeling of the physics processes for $t \bar{t}$ production and background, modeling of the detector performance and uncertainties in the method itself. There are two different types of systematics. The first is where we can associate the uncertainty with a well defined $1 \sigma$ uncertainty in a given parameter. In this case, we vary that parameter by its uncertainty and measure the resulting change in the top mass. The second is where we do not have a well defined $1 \sigma$ shift in a parameter. In this case, we generate a number of alternatives which we hope cover the space of possibilities and take the largest difference between the nominal mass and the various alternatives as our systematic shift. 
A brief introduction to the main sources of systematic uncertainties for our analysis is given below.

\subsubsection{Signal and Background Modeling}

The top quark decay properties are well known in the SM, including the subsequent decay of the $W$ boson into partons, since these decays are governed by the weak interaction and the top quark does not hadronize. Furthermore, the mass, width, and branching fractions of the $W$ are known precisely and the associated uncertainties can be neglected. In contrast, significant uncertainties do arise from the production of the $t \bar{t}$ pair (modeling of the PDFs and of initial-state radiation) and the formation of final-state jets (final-state radiation, fragmentation, and hadronization modeling) 55.

ISR and FSR Radiation off the incoming and outgoing partons may affect the top quark mass measurement. Such radiation changes the kinematics of the $t \bar{t}$ decay products in the final state; for example, the transverse momentum of the $t \bar{t}$ system is not zero when initial-state radiation (ISR) takes place. Final state radiation (FSR) changes the momenta of the $t \bar{t}$ decay products and thus affects the signal probability assigned to an event. Also, ISR and FSR may lead to jets which can be misidentified as $t \bar{t}$ decay products. ISR and FSR are governed by the same equations and are modeled in the shower evolution in the MC simulation.

Fragmentation Related to the FSR are the formation of jets in the final state and the spectra of hadrons within the jets. The fragmentation and hadronization of $b$ quark jets is particularly important. In situ calibration of the jet energy scale can largely absorb the dependence on the modeling of light $(u, d, s, c)$ quark jets. Simulations based on different fragmentation and hadronization models may predict different average energy fractions contained within the reconstructed jet. This leads to an uncertainty on the relation between jet and parton energies and thus on the measured top quark mass.

Underlying Event In principle, particles produced from the remnants of the colliding hadrons may contribute energy to the jets reconstructed in the detector. It is therefore necessary to measure the average contribution and subtract it from the jet energies. The resulting uncertainty is small and usually it is included in the jet energy scale uncertainty. 
Color Reconnection One important effect to consider is the color reconnection (CR) occurring in events with multiple hard subcollisions due to color string formations between final partons from independent hard scatterings. This effect can modify the final state in the $t \bar{t}$ process. The effect of $\mathrm{CR}$ is subject to theoretical uncertainties and is accounted for differently in different $\mathrm{MC}$ codes. The difference induced on the reconstructed top quark mass measures the associated systematic error. We assign a CR systematic uncertainty to our measurement because our benchmark MC for the signal (PYTHIA) does not take CR into account. Using POWHEG samples (which accounts for CR), thus allows to reduce the systematic uncertainties.

Pile Up Multiple hadron interaction (also known as pile up) represents the systematic associated with our modeling of multiple $p \bar{p}$ interactions in a single event. The number of primary vertices in the $p \bar{p}$ collision depends on the instantaneous luminosity during the data-taking runs. Our corrections are computed in simulations which depend on run luminosity, where the event pile up generates a run-dependent number of reconstructed event vertices. The difference from the number of vertices observed in the actual data monitors the associated systematic errors.

The run-dependent CDF MC set used in earlier measurements was not produced with the same run list as the data (was covering shorter time periods and thus lower instantaneous luminosities, with fewer primary vertices than the data). For our analysis we have MC samples produced with the final run lists, covering all luminosities, so the pile-up systematic uncertainty will also be reduced.

b-jet energy scale We have assumed that the JES is the same for all jets. However, there is an additional uncertainty arising from differences between $b$ and light jets. It is possible to identify three sources of uncertainty: one is due to the uncertainty on the semileptonic decay ratio; the second is related to the uncertainty in the $b$-fragmentation modeling; the last one is due to uncertainty in the calorimeter response. Since the charged particle fraction and momentum spectrum of $b$-jets is different from that of light jets, the calorimeter response may be different.

PDFs Our analysis uses parton distribution functions giving the probability distribution for the momentum of the incoming partons. Naturally, there is some uncertainty in the determination of these PDFs, which results in a systematic uncertainty in our measurement. 


\subsubsection{Modeling of Detector Response}

The dominant error in the top quark mass measurement is due to uncertainties in the detector response. Despite the possibility of in situ JES calibration, the absolute jet energy scale uncertainty still dominates the world average.

Residual JES Even if in our analysis we apply in situ calibration, only one overall jet energy scale factor is determined. Any discrepancy between data and simulation other than such a global scale difference leads to an additional uncertainty on the top quark mass, which is however much smaller than that arising from the overall absolute calibration. Uncertainties on residual $|\eta|$ and $p_{T}$ dependencies of the jet energy scale are taken from the external calibration. Additionally, although our jets are only corrected to level 5 , we need to account for the systematic uncertainties in all levels of the jet corrections.

Event Selection Uncertainties in the event selection efficiency, notably energy-dependent effects, can lead to systematic effects on the top quark mass. For example, uncertainties on the trigger selection can introduce a bias on the selected sample propagating to the top quark mass result. Similarly, the $b$-tagging efficiencies are determined from the data and should be varied within their uncertainty in order to determine the residual systematic error.

Lepton $p_{T}$ There is also a systematic uncertainty on the lepton energy scale, although it is much simpler than the uncertainty for the jet energy scale.

\subsubsection{Measurement Method}

The calibration of the measurement method depends slightly on the $t \bar{t}$ fraction in the selected event sample. The uncertainty on this fraction leads to a systematic error on the top quark mass. Furthermore, since we use the calibration results to correct our final measurement, any uncertainty in these results naturally translates into an uncertainty on the top mass. Experience from previous analyses indicates that uncertainties of this type usually are not dominant.

In order to evaluate the contribution of each uncertainty source, we make use of appropriate MC simulations. In the present work we are going to produce custom samples, additional to those of previous CDF analyses, with the aim 
to improve the estimate of the overall uncertainty. This is a fundamental point of our analysis, since our main purpose is to reduce the uncertainties and reach a total error of about $0.6 \mathrm{GeV}$, about $20 \%$ less than the present error of the world-averaged mass value. 


\section{Conclusions}

Since the top quark mass is a fundamental parameter of the SM, the CDF Collaboration has decided to make a major effort in order to produce its most precise measurement as a "legacy" of the experiment. We expect that the new CDF top mass measurement will contribute clearing the existing tension between the latest $\mathrm{D} \emptyset$ result $[20$ and CMS result [14] which are supposed to be the most precise measurements ever obtained for the top quark mass but are rather inconsistent with each other. Hopefully a new very precise result may have a key role in the future world combination of the top quark mass measurements.

This thesis presents the current status of the analysis focusing on the contributions given by the candidate to the long preparation work needed to perform the measurement.

We investigate the lepton + jets channel with the full integrated luminosity of Run II $\left(9.0 \mathrm{fb}^{-1}\right)$. Our analysis uses the Matrix Element method to calculate a $t \bar{t}$ likelihood as a bi-dimensional function of the assumed top mass $m_{t}$ and of $\Delta_{J E S} . \Delta_{J E S}$ parametrizes the uncertainty in our knowledge of the jet energy scale. By introducing this parameter into the likelihood, we can use as a constraint the known $W$ mass to determine the optimal $\Delta_{J E S}$ and thereby reduce the final systematic error on the measured top quark mass.

For the first time in CDF, we include the background ME modeling in the likelihood integration. The massive calculations required by this double ME method imposed to develop an unconventional integration method over the phase space of the events kinematics. In order to evaluate the multidimensional integrals, we employ the Quasi-Monte Carlo technique, which significantly reduces the time required to integrate an event.

With respect to the previous CDF top mass analysis performed using the ME method [11], we augment the statistics by including new event categories and adopting wider cuts, and employ upgraded MC codes and detector modeling. With these improvements we expect to significantly reduce the systematic error of the final result.

Preliminarily to the final analysis of real data, future studies will include 
the calibration procedure by means of pseudo-experiments and the production of new MC samples for the evaluation of systematic errors. The goal of the measurement is to reach a total error of less than $0.5 \%$. The candidate is planning to contribute from a distance to the final part of the measurement. 


\section{Bibliography}

[1] 2016. URL: http : / / project-gfitter . web . cern . ch / projectgfitter/Standard_Model/.

[2] R. Barlow. "Application of the bootstraps resampling technique to particle physics experiments". In: Manchester Part. Phys. MAN/HEP/99/4 (2000). URL: http : / / www . hep . manchester . ac . uk/preprints / manhep99-4.ps.

[3] N. Cabibbo, L. Maiani, and O. Benhar. Introduzione alle teorie di gauge. Editori Riuniti, 2016.

[4] J. Cheng and M. J. Druzdzel. "Computational Investigation of LowDiscrepancy Sequences in Simulation Algorithms for Bayesian Networks". In: arXiv:1301.3841 (2000). URL: http : / / arxiv . org/pdf / 1301 $3841 \mathrm{v} 1 . \mathrm{pdf}$.

[5] ATLAS Collaboration. "Observation of a new particle in the search for the Standard Model Higgs boson with the ATLAS detector at the LHC". In: Phys. Rev. Lett. B716 (2012), pp. 1-29. URL: http: //arxiv.org/abs/1207.7214.

[6] CDF Collaboration. "Measurement of the $t \bar{t}$ production cross section in $p \bar{p}$ collisions at $\sqrt{s}=1.96 \mathrm{TeV}$ using kinematic fitting of b-tagged lepton+jet events". In: Phys. Rev. D 71, 072005 (2005).

[7] CDF Collaboration. "Observation of Electroweak Single Top-Quark Production". In: Phys. Rev. Lett. 103 (2009).

[8] CDF Collaboration. "Observation of Top Quark Production in $p \bar{p}$ Collisions with the Collider Detector at Fermilab". In: Phys. Rev. Lett. 74.2626 (1995). URL: http://arxiv .org/pdf /hep-ex/9503002v2 pdf.

[9] CDF Collaboration. "Precision Top-Quark Mass Measurement at CDF". In: Phys. Rev. Lett. 109.152003 (2012). URL: http://arxiv.org/pdf/ 1207.6758v1.pdf. 
[10] CDF Collaboration. "The CDF II Detector Technical Design Report". In: Fermilab Publication FERMILAB-PUB-96/390-E (1996).

[11] CDF Collaboration. "Top Quark Mass Measurement in the Lepton + Jets Channel Using a Matrix Element Method and in situ Jet Energy Calibration". In: Phys.Rev.Lett. 105.252001 (2010). URL: http://arxiv org/pdf/1010.4582v2.pdf.

[12] CDF Collaboration and D0 Collaboration. "Combination of measurements of the top-quark pair production cross section from the Tevatron Collider". In: Phys. Rev. D 89, 072001 (2014). URL: http://arxiv org/abs/1309.7570.

[13] CDF Collaboration and D0 Collaboration. "Tevatron Combination of Single-Top-Quark Cross Sections and Determination of the Magnitude of the Cabibbo-Kobayashi-Maskawa Matrix Element $V_{t b}$ ". In: Phys. Rev. Lett. 115, 152003 (2015). URL: http://arxiv.org/abs/1503.05027.

[14] CMS Collaboration. "Measurement of the top quark mass using protonproton data at $\sqrt{s}=7$ and $8 \mathrm{TeV}$ ". In: arXiv:1509.04044 (2015). URL: http://www.arxiv.org/abs/1509.04044

[15] CMS Collaboration. "Observation of a new boson at a mass of 125 GeV with the CMS experiment at the LHC". In: Phys. Rev. Lett. B 716 (2012), p. 30. URL: http://arxiv.org/abs/1207.7235.

[16] D0 Collaboration. "A precision measurement of the mass of the top quark". In: Nature 429 (2004), pp. 638-642.

[17] D0 Collaboration. "Helicity of the W boson in lepton + jets $t \bar{t}$ events". In: Phys. Lett. B 617.1 (2005).

[18] D0 Collaboration. "Observation of Single Top-Quark Production". In: Phys. Rev. Lett. 103 (2009).

[19] D0 Collaboration. "Observation of the Top Quark". In: Phys. Rev. Lett. 74.2632 (1995). URL: http://arxiv.org/abs/hep-ex/9503003.

[20] D0 Collaboration. "Precision measurement of the top quark mass in lepton+jets final states". In: arXiv:1501.07912v2 (2015). URL: http: //arxiv.org/pdf/1501.07912v2.pdf.

[21] ATLAS/CDF/CMS/D0 Collaborations. "First combination of Tevatron and LHC measurements of the top-quark mass". In: LHC/Tevatron note (2014). URL: http://arxiv.org/abs/1403.4427.

[22] G. Cowan. Statistical Data Analysis. Oxford Science Publications, 1998. 
[23] L. Demortier and L. Lyons. "Everything you always wanted to know about pulls". In: $C D F / A N A L / P U B L I C / 5776$ (2002). URL: http:// physics . rockefeller . edu / luc/technical_ reports / cdf5776 _ pulls.pdf.

[24] Fermilab Accelerator Division. "Accelerator Concepts". In: (2010). URL: http://operations.fnal.gov/rookie_books/Concepts_v3.6.pdf.

[25] Fermilab Accelerator Division. "Run II hand book". In: (2003). URL: http://www-ad.fnal.gov/runII/index.html.

[26] Fermilab Accelerator Division. "The Antiproton Source rookie book". In: (2011). URL: http://operations.fnal.gov/rookie_books/Pbar_ $\mathrm{v} 2.2 . \mathrm{pdf}$.

[27] Fermilab Accelerator Division. "The Booster rookie book". In: (2009). URL: http: //operations.fnal.gov/rookie_books/Booster_V4.1 pdf.

[28] Fermilab Accelerator Division. "The Linac rookie book". In: (2006). URL: http://operations.fnal.gov/rookie_books/LINAC_RB_v2_3.pdf.

[29] Fermilab Accelerator Division. "The Main Injector rookie book". In: (2010). URL: http://operations . fnal . gov/rookie_books/Main_ Injector_v1.1.pdf.

[30] Fermilab Accelerator Division. "The Recycker Ring rookie book". In: (2010). URL: http://operations .fnal.gov/rookie_books/Recycler. RB_v1.42.pdf.

[31] Fermilab Accelerator Division. "The Tevatron rookie book". In: (2009). URL: http://operations.fnal.gov/rookie_books/Tevatron_v2.3 pdf.

[32] K.A. Olive et al. (Particle Data Group). "Review of particle physics". In: Chin. Phys. C 38.090001 (2014 and 2015 update). URL: http: //pdg.lbl.gov.

[33] A. Barbaro Galtieri et al. "Precision measurement of the top quark mass from the Tevatron in the pre-LHC era". In: Rept. Prog. Phys. 75.5 (2012). URL: http://arxiv.org/abs/1109.2163.

[34] A. Bhatti et al. "Determination of the Jet Energy Scale at the Collider Detector at Fermilab". In: Nucl. Instrum. Methods A.566 (2006), p. 375. URL: https://arxiv.org/pdf/hep-ex/0510047v1.pdf.

[35] D. Acosta et al. "The performance of the CDF Luminosity Monitor". In: Nucl. Instrum. Methods A.494 (2002), pp. 57-62. URL: http://wwwcdf.fnal.gov/ konigsb/instr02.ps. 
[36] F. A. Berends et al. "On the production of a W and jets at hadron colliders". In: Nucl. B.357 (1991), pp. 32-64. URL: http://inspirehep net/record/300269.

[37] F. Abe et al. "Topology of three-jet events in $p \bar{p}$ collisions at $\sqrt{s}=1.8$ TeV". In: Phys. Rev. D.45 (1992), p. 1448.

[38] F. Fiedler et al. "The Matrix Element Method and its Application to Measurements of the Top Quark Mass". In: Nucl. Instrum. Meth. A.624 (2010), pp. 203-218. URL: http://arxiv.org/abs/1003.1316.

[39] G. Apollinari et al. "Shower maximum detector for the CDF plug upgrade calorimeter". In: Nucl. Instrum. Methods A.412 (1998), p. 512.

[40] G. Ascoli et al. "CDF central muon detector". In: Nucl. Instrum. Methods A.268 (1988), p. 33.

[41] G. Corcella et al. "HERWIG 6.5: an event generator for Hadron Emission Reactions With Interfering Gluons (including supersymmetric processes)". In: JHEP (2001).

[42] H. L. Lai et al. "Global QCD". In: Eur. Phys. J. C 12.375 (2000). URL: http://arxiv.org/pdf/hep-ph/9903282v3.pdf.

[43] H.S. Lee et al. "Top quark mass measurement in the Lepton+Jets channnel using 8.7fb ${ }^{-1}$ data". CDF Note 10749. 2012.

[44] J. Adelman et al. "Method II For You". CDF Note 9185. May 2006. URL: http://www-cdf .fnal .gov/cdfnotes/cdf9185_Method_II_ For_You.pdf.

[45] J. Alwall et al. "MadGraph 5: Going Beyond". In: JHEP 1106 (2011), p. 128. URL: http://arxiv.org/abs/1106.0522.

[46] J. Alwall et al. "MadGraph MadEvent v4: The New Web Generation". In: JHEP 0709.028 (2007). URL: http://arxiv.org/pdf/0706.2334v1 pdf.

[47] L. Balke et al. "The CDF Central Electromagnetic Calorimeter". In: Nucl. Instrum. Methods A.267 (1988), p. 272. URL: http://inspirehep.net/ record/22817/files/fermilab-pub-87-172-E.pdf?version=1.

[48] M. L. Mangano et al. "ALPGEN, a generator for hard multiparton processes in hadronic collisions". In: JHEP 0307.001 (2003). URL: http: //arxiv.org/pdf/hep-ph/0206293v2.pdf

[49] M.Albrow et al. "The CDF plug upgrade electromagnetic calorimeter: test beam results," in: Nucl. Instrum. Methods A.480 (2002), p. 524. 
[50] S. Bertolucci et al. "The CDF Central and Endwall Hadron Calorimeter". In: Nucl. Instrum. Methods A.267 (1988), p. 301. URL: http: //inspirehep.net/record/251409/files/fermilab-pub-87-174E.pdf?version=1.

[51] S. Cabrera et al. "The CDF Time of Flight Detector". In: FERMILABConf-03/404-E (2004). URL: http://Iss.fnal.gov/archive/2003/ conf/fermilab-conf-03-404-e.pdf.

[52] S.W. Herb et al. "Observation of a Dimuon Resonance at $9.5 \mathrm{GeV}$ in 400-GeV Proton-Nucleus Collisions". In: Phys. Rev. Lett. 39.252 (1977). URL: http://inspirehep .net/record/120368/files/pub-77-058EXP.pdf?version=1.

[53] T. Aaltonen et al. "Operational experience, improvements, and performance of the CDF Run II silicon vertex detector". In: Nuclear Instruments and Methods in Physics Research A.729 (2013), pp. 153-181. URL: http://arxiv.org/pdf/1301.3180v3.pdf.

[54] T. Affolder et al. "CDF Central Outer Tracker". In: Nucl. Instrum. Meth A.526 (2004), pp. 249-299. URL: http://www.phy.ncu.edu.tw/ syu/ papers/NIMA526.249.pdf.

[55] F. Fiedler. "Precision Measurements of the Top Quark Mass". PhD thesis. Ludwig-Maximilians-Universitat Munchen, 2007. URL: http: //arxiv.org/pdf/1003.0521v1.pdf.

[56] F.J.Hickernell and L.A.Jiménez Rugama. "Reliable adaptive cubature using digital sequences". In: R. Cools, D. Nuyens (eds.) Monte Carlo and Quasi-Monte Carlo Methods (2014). URL: http://arxiv.org/ $\mathrm{pdf} / 1410.8615 \mathrm{v} 1 . \mathrm{pdf}$.

[57] CDF Internal. Method II For You Tables. URL: http://www-cdf .fnal gov/ hslee/internal/M24U/P38/.

[58] F. Jegerlehner. "On the difference between the pole and the $\overline{M S}$ masses of the top quark at the electroweak scale". In: Phys. Lett. B (2012). URL: http://arxiv.org/pdf/1212.4319v2.pdf.

[59] N. Kidonakis. "Differential and total cross sections for top pair and single top production". In: arXiv:1205.3453 (2012). URL: https:// arxiv.org/pdf/1205.3453v1.pdf.

[60] K. Kondo. "Dynamical Likelihood Method for Reconstruction of Events with Missing Momentum". In: J. Phys. Soc. Jap. 57, 412657.12 (1988), pp. 4126-4140. 
[61] P. J. Lujan. "Precision Measurement of the Top Quark Mass in the Lepton + Jets Channel Using a Matrix Element Method with QuasiMonte Carlo Integration". PhD thesis. Harvard University, 2000. URL: http://www-cdf.fnal.gov/thesis/cdf9908_plujan_thesis.pdf.

[62] D. Mohl. "Physics and technique of stochastic cooling". In: Phys. Reports 58 (1980).

[63] W.J. Morokoff and R. E. Caflisch. "Quasi-Monte Carlo Integration”. In: Journal of computational physics 122 (1995), pp. 218-230.

[64] P. Nason. "A New Method for Combining NLO QCD with Shower Monte Carlo Algorithms". In: JHEP 0411.040 (2004). URL: http:// arxiv.org/pdf/hep-ph/0409146v1.pdf.

[65] C. Neu. "CDF b-tagging: Measuring Efficiency and False Positive Rate". In: Proceeding Of Science (2006). URL: http://pos . sissa it/archive/conferences/024/015/TOP2006_015.pdf.

[66] D. H. Perkins. Introduction to High Energy Physics. Cambridge University Press, 2000.

[67] D0 public page. Useful Diagrams of Top Signals and Background. URL: http : / / www - d0 . fnal . gov/Run2Physics/top/top_public_web_ pages/top_feynman_diagrams.html.

[68] R.Kleiss and W.J.Stirling. "Top quark production at hadron colliders: some useful formulae". In: Z.Phys.C-Particle and Fields 40 (1988), pp. 419-423.

[69] L.A.Jimenez Rugama. MTM3 elog, entries n. 79, 80, 84, 85. URL: http://tdpc681.fnal.gov:8080/MTM3/.

[70] T. Sjöstrand, L. Lönnblad, and S. Mrenna. "PYTHIA 6.2 Physics and Manual". In: Computer Physics Commun 135.238 (2001). URL: http://arxiv.org/pdf/hep-ph/0108264v1.pdf.

[71] T. Teubner. "The Standard Model". In: Lecture presented at the School for Experimental High Energy Physics Students Somerville College, Oxford (2009). URL: http://www.ppd.stfc.ac.uk/ppd/resources/ pdf/standardmodel09.pdf.

[72] Wikipedia the free encyclopedia. Standard Model. URL: https://en wikipedia.org/wiki/Standard_Model.

[73] I. Volobouev. "JES-Related Transfer Function Details". In: CDF Internal (2014). URL: http://tdpc681.fnal.gov:8080/MTM3/150629_151347/ tf_details.pdf. 
[74] I. Volobouev. "Matrix Element Method in HEP: Transfer Functions, Efficiencies, and Likelihood Normalization". In: arXiv:1101.2259v1 (2011). URL: http://arxiv.org/pdf/1101.2259v1.pdf. 